\title{
A NEW LOOK AT DUALITY FOR THE SYMBIOTIC BRANCHING MODEL ${ }^{1}$
}

\author{
By Matthias Hammer*, MARCEl ORTGiese ${ }^{\dagger}$ AND Florian Völlering $^{\dagger}$ \\ Technische Universität Berlin* and University of Bath ${ }^{\dagger}$
}

The symbiotic branching model is a spatial population model describing the dynamics of two interacting types that can only branch if both types are present. A classical result for the underlying stochastic partial differential equation identifies moments of the solution via a duality to a system of Brownian motions with dynamically changing colors. In this paper, we revisit this duality and give it a new interpretation. This new approach allows us to extend the duality to the limit as the branching rate $\gamma$ is sent to infinity. This limit is particularly interesting since it captures the large scale behavior of the system. As an application of the duality, we can explicitly identify the $\gamma=\infty$ limit when the driving noises are perfectly negatively correlated. The limit is a system of annihilating Brownian motions with a drift that depends on the initial imbalance between the types.

\section{CONTENTS}

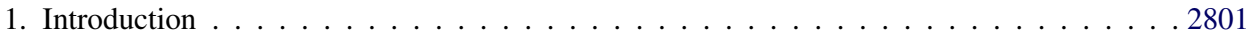

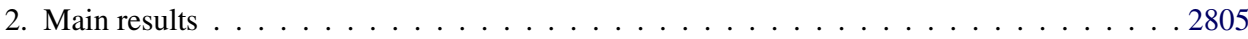

2.1. Moment duality for $\operatorname{SBM}(\varrho, \infty) \ldots \ldots \ldots \ldots \ldots \ldots \ldots$

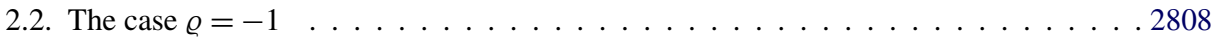

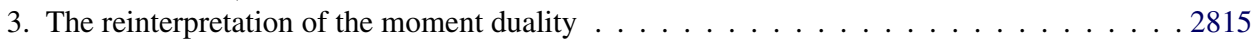

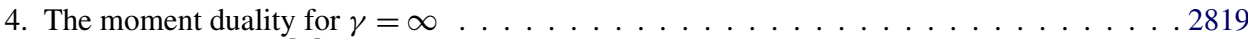

4.1. Analysis of $M^{[\gamma]}$ for the discrete space model . . . . . . . . . . . . . . . . . 2819

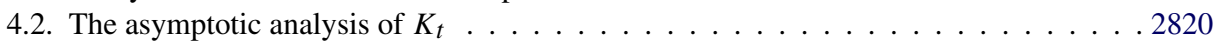

4.3. Proof of Theorem 2.3 in the discrete case . . . . . . . . . . . . . . . . . . . . 2827

4.4. Proof of Theorem 2.3 in the continuous case . . . . . . . . . . . . . . . . . . . 2827

4.5. Proof of Theorem $2.5 \ldots \ldots$. . . . . . . . . . . . . . . . . . . . . . . . . . . . . 2829

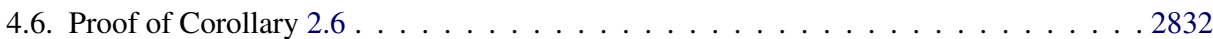

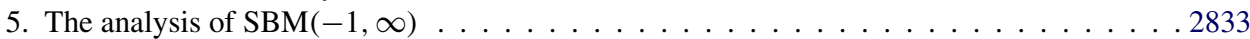

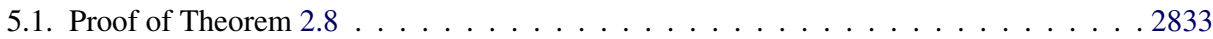

5.2. Some notation and preliminaries . . . . . . . . . . . . . . . . . . . 2842

5.3. Non-proliferation of interfaces . . . . . . . . . . . . . . . . . . . . . . . . . . . 2844

5.4. Movement of a single interface . . . . . . . . . . . . . . . . . . . . . . . . 2846

5.5. Multiple interfaces . . . . . . . . . . . . . . . . . . . . . . 2852

5.6. General initial configurations . . . . . . . . . . . . . . . . . . 2858

Received September 2016; revised October 2017.

${ }^{1}$ Supported by the German Research Foundation (DFG) within the DFG Priority Programme 1590 "Probabilistic Structures in Evolution", Grants no. BL 1105/4-1 and OR 310/1-1.

MSC2010 subject classifications. Primary 60K35; secondary 60J80, 60H15.

Key words and phrases. Symbiotic branching model, mutually catalytic branching, stepping stone model, rescaled interface, moment duality, annihilating Brownian motions. 
1. Introduction. In [10], Etheridge and Fleischmann introduce a spatial population model that describes the evolution of two interacting types. The dynamics follows locally a branching process, where each type branches with a rate proportional to the frequency of the other type. Additionally, types are allowed to migrate to neighbouring colonies. In the continuum space and large population limit, the symbiotic branching model is the process $\left(\mathbf{u}_{t}\right)_{t \geq 0}=\left(u_{t}^{(1)}, u_{t}^{(2)}\right)_{t \geq 0}$, where the frequencies $u_{t}^{(1)}(x)$ and $u_{t}^{(2)}(x)$ of the respective types are given by the nonnegative solutions of the stochastic partial differential equations

$$
\operatorname{cSBM}(\varrho, \gamma)_{\mathbf{u}_{0}}:\left\{\begin{array}{l}
\frac{\partial}{\partial t} u_{t}^{(1)}(x)=\frac{\Delta}{2} u_{t}^{(1)}(x)+\sqrt{\gamma u_{t}^{(1)}(x) u_{t}^{(2)}(x)} \dot{W}_{t}^{(1)}(x), \\
\frac{\partial}{\partial t} u_{t}^{(2)}(x)=\frac{\Delta}{2} u_{t}^{(2)}(x)+\sqrt{\gamma u_{t}^{(1)}(x) u_{t}^{(2)}(x)} \dot{W}_{t}^{(2)}(x),
\end{array}\right.
$$

with suitable nonnegative initial condition $\mathbf{u}_{0}=\left(u_{0}^{(1)}, u_{0}^{(2)}\right), u_{0}^{(i)}(x) \geq 0, x \in \mathbb{R}$, $i=1,2$. Here, $\gamma>0$ is the branching rate and $\left(\dot{W}^{(1)}, \dot{W}^{(2)}\right)$ is a pair of correlated standard Gaussian white noises on $\mathbb{R}_{+} \times \mathbb{R}$ with correlation governed by a parameter $\varrho \in[-1,1]$, that is,

$$
\mathbb{E}\left[\dot{W}_{t_{1}}^{(1)}\left(x_{1}\right) \dot{W}_{t_{2}}^{(2)}\left(x_{2}\right)\right]=\varrho \delta_{0}\left(t_{1}-t_{2}\right) \delta_{0}\left(x_{1}-x_{2}\right), \quad t_{1}, t_{2} \geq 0, x_{1}, x_{2} \in \mathbb{R},
$$

where $\delta_{0}$ denotes the delta function at 0 .

There is also a discrete-space version of the model on the lattice $\mathbb{Z}^{d}$, where $\frac{1}{2} \Delta$ is replaced by the discrete Laplacian

$$
\Delta^{d} f(x)=\frac{1}{2 d} \sum_{y \sim x}(f(y)-f(x)),
$$

where $y \sim x$ indicates that $x$ and $y$ are neighbors on $\mathbb{Z}^{d}$ and $f: \mathbb{Z}^{d} \rightarrow \mathbb{R}$. Moreover, in this case the white noises are replaced by an independent system $\left(W^{1}(x), W^{2}(x)\right), x \in \mathbb{Z}^{d}$, of $\varrho$-correlated two-dimensional Brownian motions. We will refer to the discrete space model with initial condition $\mathbf{u}_{0}$ as $\operatorname{dSBM}(\varrho, \gamma)_{\mathbf{u}_{0}}$. Existence (for $\varrho \in[-1,1]$ ) and uniqueness (for $\varrho \in[-1,1)$ ) in both continuous and discrete space was proved in [10] for a large class of initial conditions.

The symbiotic branching model generalizes several well-known examples of spatial populations dynamics. In particular, for the case $\varrho=-1$ of perfectly negatively correlated noises, the sum $u_{t}^{(1)}+u_{t}^{(2)}$ solves the (deterministic) heat equation, so we have $u_{t}^{(1)}+u_{t}^{(2)}=S_{t}\left(u_{0}^{(1)}+u_{0}^{(2)}\right)$ for all $t \geq 0$, with $\left(S_{t}\right)_{t \geq 0}$ denoting the heat semigroup. In particular, for initial conditions $u_{0}^{(1)}=1-u_{0}^{(2)}$ summing up to one, we have $u_{t}:=u_{t}^{(1)}=1-u_{t}^{(2)}$ for all $t \geq 0$, and the system reduces to the continuous-space stepping stone model

$$
\frac{\partial}{\partial t} u_{t}(x)=\frac{\Delta}{2} u_{t}(x)+\sqrt{\gamma u_{t}(x)\left(1-u_{t}(x)\right)} \dot{W}_{t}(x)
$$


analysed, for example, in [22] and [24]. For $\varrho=0$, the system is known as the mutually catalytic branching model due to Dawson and Perkins [6].

We are particularly interested in the case where initially both types are spatially separated. In the simplest case, this corresponds to starting the system with "complementary Heaviside initial conditions" $\mathbf{u}_{0}=\left(u_{0}^{(1)}, u_{0}^{(2)}\right)=\left(\mathbb{1}_{\mathbb{R}^{-}}, \mathbb{1}_{\mathbb{R}^{+}}\right)$. Then one would like to understand the evolution of the interface between the two types, which is defined by

$$
\operatorname{Ifc}_{t}=\operatorname{cl}\left\{x \in \mathbb{R}: u_{t}^{(1)}(x) u_{t}^{(2)}(x)>0\right\},
$$

where $\mathbf{u}_{t}=\left(u_{t}^{(1)}, u_{t}^{(2)}\right)$ is the solution of $\operatorname{cSBM}(\varrho, \gamma)_{\mathbf{u}_{0}}$ at time $t>0$, and $\operatorname{cl}(A)$ denotes the closure of the set $A$ in $\mathbb{R}$. Results on the growth of this interface due to [10], [3] suggest diffusive behavior for the interface. This conjecture is supported by the following scaling property of the model; see [10], Lemma 8: Let $\left(\mathbf{u}_{t}\right)_{t \geq 0}$ denote the solution to $\operatorname{cSBM}(\varrho, \gamma) \mathbf{u}_{0}$. If we rescale time and space diffusively, that is: If given $K>0$, we define

$$
\mathbf{v}_{t}^{[K]}(x):=\mathbf{u}_{K^{2} t}(K x), \quad x \in \mathbb{R}, t \geq 0,
$$

then $\left(\mathbf{v}_{t}^{[K]}\right)_{t \geq 0}$ is a solution to $\operatorname{cSBM}(\varrho, K \gamma)_{\mathbf{v}_{0}^{[K]}}$, that is, a symbiotic branching process with branching rate $K \gamma$ and correspondingly transformed initial states $\mathbf{v}_{0}^{[K]}(x)=\mathbf{u}_{0}(K x), x \in \mathbb{R}$. Thus, provided that the initial conditions are invariant under diffusive rescaling (as is the case for complementary Heaviside), this rescaling of the system is equivalent (in law) to increasing the branching rate. This observation suggests to investigate an infinite rate limit $\gamma \rightarrow \infty$, which can also be considered for more general initial conditions.

This program has been carried out first for the discrete-space model $\mathrm{dSBM}(\varrho, \gamma)$, also inspired by the scaling property of the continuous model. For $\varrho \in(-1,1)$ and suitable initial conditions $\mathbf{u}_{0}=\left(u_{0}^{(1)}, u_{0}^{(2)}\right)$ which are mutually singular, that is, $u_{0}^{(1)}(x) u_{0}^{(2)}(x)=0$ for all $x \in \mathbb{Z}^{d},[8,9,17-19]$ construct a non-trivial limiting process of $\operatorname{dSBM}(\varrho, \gamma)_{\mathbf{u}_{0}}$ as $\gamma \rightarrow \infty$ and study its long-term properties. They also give a very explicit description of the limit in terms of an infinite system of jumptype stochastic differential equations (SDEs). The limit corresponds to a system where the two types remain separated, that is, at each lattice point only one type is present. We will refer to the limit as the discrete-space infinite rate symbiotic branching model, abbreviated as $\operatorname{dSBM}(\varrho, \infty)$.

For the continuous space model $\operatorname{cSBM}(\varrho, \gamma)$, an infinite rate limit has been shown to exist recently in [4] for the case of negative correlations $\varrho \in(-1,0)$ and a large class of initial conditions. More precisely, it was proved that the measure-valued processes obtained by taking the solutions of $\operatorname{cSBM}(\varrho, \gamma)_{\mathbf{u}_{0}}$ as densities converge in law as $\gamma \uparrow \infty$ to a measure-valued process $\left(\mu_{t}^{(1)}, \mu_{t}^{(2)}\right)_{t \geq 0}$ also satisfying a certain separation-of-types condition; see [4], Theorem 1.10. We 
call the limit $\left(\mu_{t}^{(1)}, \mu_{t}^{(2)}\right)_{t \geq 0}$ the continuous-space infinite rate symbiotic branching model $\operatorname{cSBM}(\varrho, \infty)$. As for the discrete case, the convergence is generally in the Meyer-Zheng "pseudo-path" topology on $D_{[0, \infty)}$, which is strictly weaker than the standard Skorokhod topology. Under the more restrictive condition that $\mathbf{u}_{0}=\left(\mathbb{1}_{\mathbb{R}^{-}}, \mathbb{1}_{\mathbb{R}^{+}}\right)$and $\varrho \in\left(-1,-\frac{1}{\sqrt{2}}\right)$, it is possible to show convergence in the stronger Skorokhod topology on $\mathcal{C}_{[0, \infty)}$; see [4], Theorems 1.5, 1.12. Also, in this case the limiting measures $\mu_{t}^{(1)}$ and $\mu_{t}^{(2)}$ are absolutely continuous with respect to Lebesgue measure and mutually singular, that is, we have

$$
\mu_{t}^{(1)}(\cdot) \mu_{t}^{(2)}(\cdot) \equiv 0 \quad \text { almost surely. }
$$

In [4], $\operatorname{cSBM}(\varrho, \infty)$ is characterized in terms of a rather abstract martingale problem. In contrast to the discrete-space case (see [18]), a fully explicit characterization of the continuous-space limit is still missing so far. Recently, in [13] the gap between the two infinite rate models has been narrowed somewhat by showing that for all $\varrho \in(-1,0), \operatorname{dSBM}(\varrho, \infty)$ converges to $\operatorname{cSBM}(\varrho, \infty)$ under diffusive rescaling. This opens up the possibility to obtain results on the continuous model from analogous ones for the discrete model. For example, it was shown in [13] that for complementary Heaviside initial conditions, for each fixed time $t>0$, almost surely there exists a single interface, that is a point which is separating the two types.

The convergence result in [4] does not include the case $\varrho=-1$. In this case, for complementary Heaviside initial conditions, a diffusive scaling limit was already proved in [24] for the continuum stepping stone model, as one of the steps of understanding the diffusively rescaled interface. Under this assumption, it was shown that the measure-valued limit $\left(\mu_{t}^{(1)}, \mu_{t}^{(2)}\right)_{t \geq 0}$ has the law of

$$
\left(\mathbb{1}_{\left\{x \leq B_{t}\right\}} d x, \mathbb{1}_{\left\{x \geq B_{t}\right\}} d x\right)_{t \geq 0},
$$

for $\left(B_{t}\right)_{t \geq 0}$ a standard Brownian motion. More generally, instead of just complementary Heaviside [24] considered also initial conditions with "multiple interfaces", but still under the assumption that they sum up to one so that the system reduces to the stepping stone model (1). It was shown that these solutions with a finite number of interfaces converge to a system of annihilating Brownian motions.

The original motivation for this work was the question what happens for $\varrho=-1$ and general initial conditions. This includes the case where $\mathbf{u}_{0}$ is still complementary but does not satisfy $u_{0}^{(1)}+u_{0}^{(2)} \equiv 1$. This case corresponds also to a stepping stone model, but where we do not consider relative frequencies and instead absolute numbers (and then densities). Locally, the dynamics are still given by a Wright-Fisher model, preserving the number of particles. However, now there is migration to neighboring sites rather than exchange of particles as in the original model. Our aim here is to understand how an initial imbalance in absolute numbers propagates. Another motivation is to consider "overlapping" initial configurations 
where the two populations are no longer well separated. These general initial conditions are not covered by the results in [24], and the problem is also open for the discrete model; see, for example, [8], page 43.

In fact, all tightness results obtained in [4] continue to hold for $\varrho=-1$ as well. The problem is however uniqueness, since the self-duality approach employed for the case $\varrho>-1$ in [10], [18] and [4] breaks down. The same problem arises for the finite rate model as well. Instead of using self-duality, [10] establishes uniqueness for $\operatorname{SBM}(-1, \gamma)$ using a new moment duality introduced there, which we recall below and which replaces Shiga's [22] moment duality for the stepping stone model involving coalescing Brownian motions. For $\varrho=-1$, the process is characterized by its moments precisely because the sum $u_{t}^{(1)}+u_{t}^{(2)}$ still solves the deterministic heat equation. This suggests a strategy to prove uniqueness for $\operatorname{SBM}(-1, \infty)$ by extending the moment duality of [10] to the infinite rate limit. Indeed, this (rather non-trivial) extension is one of the main results in the present work.

We now briefly recall the moment duality due to [10]. Let $\mathbf{u}_{t}=\left(u_{t}^{(1)}, u_{t}^{(2)}\right)$ denote the solution of $\operatorname{SBM}(\varrho, \gamma)$ (in discrete or continuous space) with initial condition $\mathbf{u}_{0}=\left(u_{0}^{(1)}, u_{0}^{(2)}\right)$. Let $\mathcal{S} \in\left\{\mathbb{Z}^{d}, \mathbb{R}\right\}$. Fix $n \in \mathbb{N}$. For $\mathbf{x}=\left(x_{1}, \ldots, x_{n}\right) \in \mathcal{S}^{n}$ and $c=\left(c_{1}, \ldots, c_{n}\right) \in\{1,2\}^{n}$, one is interested in the (mixed) moments

$$
\mathbb{E}_{u_{0}^{(1)}, u_{0}^{(2)}}\left[\prod_{i=1}^{n} u_{t}^{\left(c_{i}\right)}\left(x_{i}\right)\right], \quad t>0 .
$$

We can interpret the two types as "colors", thus we will call each element of $\{1,2\}$ " a coloring. In discrete resp. continuous space, the dual process is defined as follows: At time $t=0$, we start with $n$ particles located at $\mathbf{x} \in \mathcal{S}^{n}$ and colored according to $c \in\{1,2\}^{n}$, that is, $c_{i}$ is the color of particle $i$ located at $x_{i}$. The particles move on paths given by a family of independent simple symmetric random walks, resp. Brownian motions, $\left(X_{t}\right)_{t \geq 0}$ starting at $\mathbf{x}$. When two particles meet, they start collecting collision local time. If both particles are of the same color, each of them changes color when their collision local time exceeds an (independent) exponential time with parameter $\gamma / 2$, resulting in a total rate of $\gamma$ per pair of particles for a change of color. Denote by $\left(C_{t}\right)_{t \geq 0}$ the resulting coloring process of $X$, that is $C_{t} \in\{1,2\}^{n}$ prescribes the color of the $n$ particles at time $t$. Finally, denote by $L_{t}^{=}$ the total collision local time collected by all pairs of the same color up to time $t$, and let $L_{t}^{\neq}$be the collected local time of all pairs of different color up to time $t$. For a more rigorous definition of the dual process and more details, we refer to [10], Sections 3.2 and 4.1. The mixed moment duality function is given (up to an exponential correction involving $L_{t}^{=}$and $L_{t}^{\neq}$) by

$$
H(\mathbf{u} ; \mathbf{x}, c):=\mathbf{u}^{(c)}(\mathbf{x}):=\prod_{i=1}^{n} u^{\left(c_{i}\right)}\left(x_{i}\right) .
$$


Now the moment duality for $\operatorname{SBM}(\varrho, \gamma)$ reads as follows [10], Propositions 9, 12:

$$
\mathbb{E}_{\mathbf{u}_{0}}\left[H\left(\mathbf{u}_{t} ; \mathbf{x}, c\right)\right]=\mathbb{E}_{\mathbf{x}, c}\left[H\left(\mathbf{u}_{0} ; X_{t}, C_{t}\right) e^{\gamma\left(L_{t}^{=}+\varrho L_{t}^{\neq}\right)}\right] .
$$

Although the moment duality is particularly important for $\varrho=-1$ since there it is needed to ensure uniqueness, it holds for all values of $\varrho \in[-1,1]$. In [3], it was used (together with the self-duality) to investigate how the long-term behavior of the moments depends on the parameter $\varrho$. More precisely, define the critical curve $p:[-1,1) \rightarrow(1, \infty]$ of the symbiotic branching model by

$$
p(\varrho)=\frac{\pi}{\arccos (-\varrho)},
$$

and denote its inverse by $\varrho(p)=-\cos \left(\frac{\pi}{p}\right)$ for $p \in(1, \infty]$. As shown in [3], Theorem 2.5, this critical curve determines the long-term behavior of the moments of $\operatorname{SBM}(\varrho, \gamma)$ for uniform initial conditions: We have

$$
\varrho<\varrho(p) \Rightarrow \mathbb{E}_{\mathbb{1}, \mathbb{1}}\left[u_{t}^{(i)}(x)^{p}\right] \text { is bounded uniformly in all } t \geq 0, x \in \mathcal{S},
$$

for $i=1,2$, and the above condition is sharp in the recurrent case, that is, for $\mathcal{S} \in\left\{\mathbb{R}, \mathbb{Z}, \mathbb{Z}^{2}\right\}$. This suggests that the infinite rate model $\operatorname{SBM}(\varrho, \infty)$ has finite $p$ th moments for $p<p(\varrho)$, and indeed this holds true (see [13], Proposition 2.8).

In this work, we extend the moment duality from [10] to the infinite rate limit $\operatorname{SBM}(\varrho, \infty)$ for all $n \in \mathbb{N}$ such that $\varrho+\cos (\pi / n)<0$, corresponding to integer moments below the critical curve. As we will explain below, this is not straightforward and first requires a suitable reinterpretation of the moment duality (2). Indeed, it is intuitive that for $\gamma \uparrow \infty$, the color change mechanism in the dual system of colored particles described above will happen instantaneously upon the collision of two particles. However, it is not at all clear what the exponential correction term in (2) will converge to. For $\varrho=-1$, the extension to $\gamma=\infty$ establishes the uniqueness of $\operatorname{SBM}(-1, \infty)$ for general initial conditions, which was so far open in both discrete and continuous space. As a further application, we investigate the continuous space model in more detail. In fact, assuming only boundedness of the initial condition $\mathbf{u}_{0}$, we provide a complete description of $\operatorname{cSBM}(-1, \infty)_{\mathbf{u}_{0}}$ in terms of annihilating Brownian motions with drift.

2. Main results. In this section, we give a precise statement of our main results. From now on, we will write $\left(\mathbf{u}_{t}^{[\gamma]}\right)_{t \geq 0}$ for the solution to $\operatorname{SBM}(\varrho, \gamma)_{\mathbf{u}_{0}}$ (in discrete or continuous space) with initial condition $\mathbf{u}_{0}$ and finite branching rate $\gamma \in(0, \infty)$. The notation $\left(\mathbf{u}_{t}\right)_{t \geq 0}$ will usually ${ }^{2}$ be reserved for the infinite rate limit $\operatorname{SBM}(\varrho, \infty))_{\mathbf{u}_{0}}$. We recall from [4] resp. [13] that the limiting process $\left(\mathbf{u}_{t}\right)_{t \geq 0}$ takes values in $D_{[0, \infty)}\left(\mathcal{M}_{\text {tem }}(\mathcal{S})^{2}\right)$, where $\mathcal{M}_{\text {tem }}(\mathcal{S})$ denotes the space of tempered measures on $\mathcal{S} \in\left\{\mathbb{Z}^{d}, \mathbb{R}\right\}$. The topology on $\mathcal{M}_{\text {tem }}(\mathcal{S})$ can be described as follows:

\footnotetext{
${ }^{2}$ An exception is Corollary 2.6(ii).
} 
Convergence of a sequence of measures $u_{n} \rightarrow u$ in $\mathcal{M}_{\text {tem }}(\mathcal{S})$ as $n \rightarrow \infty$ means convergence of all integrals $\left\langle u_{n}, \phi\right\rangle \rightarrow\langle u, \phi\rangle$ against continuous test functions $\phi$ that decay exponentially fast at infinity. With this topology, $\mathcal{M}_{\text {tem }}(\mathcal{S})$ is a Polish space. Throughout, we will also use the Meyer-Zheng topology introduced in [21] and generalized in [20], where the path space is endowed with the topology induced by convergence in measure. For more details on these topologies, we refer the reader for example, to Appendix A.1 in [4].

In order to simplify the proofs, in this paper, we will consider only bounded initial densities $\mathbf{u}_{0}=\left(u_{0}^{(1)}, u_{0}^{(2)}\right)$.

2.1. Moment duality for $\operatorname{SBM}(\varrho, \infty)$. We return to the classical moment duality (2) for $\operatorname{SBM}(\varrho, \gamma)$ due to [10], which we would like to extend to $\gamma=\infty$. Recall that we call the elements of $\{1,2\}^{n}$ colorings. Let $\mathcal{M}\left(\{1,2\}^{n}\right)$ denote the space of measures on colorings, which can be identified with $\left(\mathbb{R}^{+}\right)^{\left(2^{n}\right)}$. Given the paths $X=\left(X_{t}^{(1)}, \ldots, X_{t}^{(n)}\right)_{t \geq 0}$ of either simple symmetric random walk in $\left(\mathbb{Z}^{d}\right)^{n}$ or Brownian motion in $\mathbb{R}^{n}$ and a measure on colorings $M_{0} \in \mathcal{M}\left(\{1,2\}^{n}\right)$, let $M_{t}^{[\gamma]} \equiv M_{t}^{[\gamma]}\left(X, M_{0}\right)$ be a process taking values in $\mathcal{M}\left(\{1,2\}^{n}\right)$ with initial state $M_{0}$ and evolution given by

$$
\begin{aligned}
d M_{t}^{[\gamma]}(b)= & \frac{\gamma \varrho}{2} \sum_{i, j=1}^{n} \mathbb{1}_{b_{i} \neq b_{j}} M_{t}^{[\gamma]}(b) d L_{t}^{i, j} \\
& +\frac{\gamma}{2} \sum_{i, j=1}^{n} \mathbb{1}_{b_{i} \neq b_{j}} M_{t}^{[\gamma]}\left(\widehat{b}^{i}\right) d L_{t}^{i, j},
\end{aligned}
$$

for $b \in\{1,2\}^{n}$. Here $\left(L_{t}^{i, j}\right)_{t \geq 0}$ are the pair local times of the motions $X$ and $\widehat{b}^{i}$ is the coloring $b$ flipped at $i$. The process $M_{t}^{[\gamma]}\left(X, M_{0}\right)$ is well defined almost surely w.r.t. the law of the random walk or Brownian motion.

THEOREM 2.1. Fix $\varrho \in[-1,1]$, and let $\mathcal{S} \in\left\{\mathbb{Z}^{d}, \mathbb{R}\right\}$. Given $n \in \mathbb{N}$ and a coloring $c \in\{1,2\}^{n}$, the original moment duality (2) can be rewritten as

$$
\mathbb{E}_{\mathbf{u}_{0}}\left[H\left(\mathbf{u}_{t}^{[\gamma]} ; \mathbf{x}, c\right)\right]=\mathbb{E}_{\mathbf{x}}\left[\sum_{b \in\{1,2\}^{n}} M_{t}^{[\gamma]}\left(X, \delta_{c}\right)(b) \mathbf{u}_{0}^{(b)}\left(X_{t}\right)\right] .
$$

REMARK 2.2. Let us remark that we have a lot of control over the process $M^{[\gamma]}$, in terms of eigenvectors and eigenvalues. The details of this are postponed to Section 4.

Our first result, which is crucial for the extension of the moment duality to $\gamma=\infty$, is that the process $\left(M_{t}^{[\gamma]}\left(X, M_{0}\right)\right)_{t \geq 0}$ converges almost surely as $\gamma \uparrow \infty$, provided $\varrho+\cos (\pi / n)<0$. 
THEOREM 2.3. Suppose that $\varrho+\cos (\pi / n)<0$. In the continuous-space case $\mathcal{S}=\mathbb{R}$, assume also that the starting point $\mathbf{x}=\left(x_{1}, \ldots, x_{n}\right) \in \mathbb{R}^{n}$ of the Brownian motion $X$ is such that no two coordinates are the same. Then as $\gamma \rightarrow \infty$, the process $\left(M_{t}^{[\gamma]}\right)_{t \geq 0}$ defined in (4) converges almost surely pointwise to a làdcàg limiting process $\left(M_{t}^{[\infty]}\right)_{t \geq 0}=\left(M_{t}^{[\infty]}\left(X, M_{0}\right)\right)_{t \geq 0}$.

REMARK 2.4. (a) The construction of the process $M^{[\infty]}$ in Theorem 2.3 is explicit, but also involved. Therefore, we postpone its description to Section 4; see, in particular Propositions 4.5 and 4.6.

(b) In continuous space, the extra condition on the starting point of the Brownian motions that $x_{i} \neq x_{j}$ for $i \neq j$ is only technical and may be removed, albeit at the expense of a much more involved proof. But since we will need the convergence only for Lebesgue-almost all starting points $\mathbf{x} \in \mathbb{R}^{n}$, we will not prove this.

Our second main result extends the moment duality (5) to the infinite rate limit. Note that we exclude $\varrho=-1$ since in this case the infinite rate limit has not yet been constructed for general initial conditions. In fact, in Section 2.2 we will use Theorems 2.1 and 2.3 to remedy this and obtain much more details about the case $\varrho=-1$. Since in continuous space the limiting process $\operatorname{cSBM}(\varrho, \infty)$ is measurevalued, instead of the 'pointwise' duality function employed in (5) we state the duality in a weak formulation.

THEOREM 2.5. Suppose $\varrho \in(-1,0)$. For $\mathcal{S} \in\left\{\mathbb{Z}^{d}, \mathbb{R}\right\}$, consider nonnegative and bounded initial densities $\mathbf{u}_{0}=\left(u_{0}^{(1)}, u_{0}^{(2)}\right)$; in the discrete-space case $\mathcal{S}=\mathbb{Z}^{d}$, assume also that $u_{0}^{(1)}$ and $u_{0}^{(2)}$ are mutually singular. Let $\left(\mathbf{u}_{t}\right)_{t \geq 0} \in$ $D_{[0, \infty)}\left(\mathcal{M}_{\text {tem }}(\mathcal{S})^{2}\right)$ denote the infinite rate limit $\operatorname{SBM}(\varrho, \infty)_{\mathbf{u}_{0}}$, and let $\left(M_{t}^{[\infty]}\right)_{t \geq 0}$ be the limiting process from Theorem 2.3. Then $\left(\mathbf{u}_{t}\right)_{t \geq 0}$ satisfies the following moment duality: For all $n \in \mathbb{N}$ such that $\varrho+\cos (\pi / n)<0$, all $t>0$, all colorings $c=\left(c_{1}, \ldots, c_{n}\right) \in\{1,2\}^{n}$ and all nonnegative test functions $0 \leq \phi \in L^{1}\left(\mathcal{S}^{n}\right)$, we have

(6) $\mathbb{E}_{\mathbf{u}_{0}}\left[\int_{\mathcal{S}^{n}} \phi(\mathbf{x}) \mathbf{u}_{t}^{(c)}(d \mathbf{x})\right]=\int_{\mathcal{S}^{n}} \phi(\mathbf{x}) \mathbb{E}_{\mathbf{x}}\left[\sum_{b \in\{1,2\}^{n}} M_{t}^{[\infty]}\left(X, \delta_{c}\right)(b) \mathbf{u}_{0}^{(b)}\left(X_{t}\right)\right] d \mathbf{x}$

and both sides are finite, where we write $\mathbf{u}_{t}^{(c)}(d \mathbf{x}):=\bigotimes_{i=1}^{n} u_{t}^{\left(c_{i}\right)}\left(d x_{i}\right)$ and the integral on the right hand side is taken w.r.t. Lebesgue measue if $\mathcal{S}=\mathbb{R}$ and w.r.t. counting measure if $\mathcal{S}=\mathbb{Z}^{d}$.

We will now state several consequences of the new moment duality (6) resp. of the reformulation of the finite rate duality (5). First of all, for the infinite rate model we can explicitly compute second mixed moments, where we recover a known identity (see [9], Theorem 1.2, for discrete space and [13] for the general 
case). Second, for the finite rate model on $\mathbb{Z}^{d}, d=1,2$, it is known that the critical curve $\varrho+\cos (\pi / n)=0$ determines which moments remain bounded in time. In dimensions where the random walk is transient, the condition $\varrho+\cos (\pi / n)<0$ is still sufficient for the uniform boundedness of $n$th moments; see [3], Theorem 2.5. However, as we will see in part (ii) of the following corollary, we can recover a weaker version of a result of [2] to see it is no longer sharp.

Corollary 2.6. (i) Let $\varrho \in(-1,0), \mathcal{S} \in\left\{\mathbb{Z}^{d}, \mathbb{R}\right\}$ and $\left(\mathbf{u}_{t}\right)_{t \geq 0}$ be the infinite rate limit $\operatorname{SBM}(\varrho, \infty) \mathbf{u}_{0}$ for nonnegative and bounded initial condition $\mathbf{u}_{0}=\left(u_{0}^{(1)}, u_{0}^{(2)}\right)$; in the discrete-space case $\mathcal{S}=\mathbb{Z}^{d}$, assume also that $u_{0}^{(1)}$ and $u_{0}^{(2)}$ are mutually singular. For second mixed moments, the duality (6) reads

$$
\begin{aligned}
\mathbb{E}_{\mathbf{u}_{0}}\left[\left\langle u_{t}^{(1)}, \phi\right\rangle\left\langle u_{t}^{(2)}, \psi\right\rangle\right] \\
\quad=\int_{\mathcal{S}^{2}} \phi(x) \psi(y) \mathbb{E}_{x, y}\left[u_{0}^{(1)}\left(X_{t}^{(1)}\right) u_{0}^{(2)}\left(X_{t}^{(2)}\right) \mathbb{1}_{t<\tau}\right] d x d y,
\end{aligned}
$$

where $\phi, \psi \in L^{1}(\mathbb{R})$ and $\tau$ denotes the first collision time of the two random walks resp. Brownian motions $\left(X^{(1)}, X^{(2)}\right)$.

(ii) [2] Let $\varrho \in[-1,1], \mathcal{S}=\mathbb{Z}^{d}$ for $d \geq 3$ and $\left(\mathbf{u}_{t}\right)_{t \geq 0}$ be the solution of $\operatorname{dSBM}(\varrho, \gamma)_{\mathbf{u}_{0}}$ for $\gamma \in(0, \infty)$ and nonnegative bounded initial condition $\mathbf{u}_{0}$. Let $p_{d}$ be the return probability of a random walk to the origin. If $\frac{\varrho \gamma}{2\left(1-p_{d}\right)}<1$, then for all $t \geq 0, x \in \mathbb{Z}^{d}$,

$$
\mathbb{E}_{\mathbf{u}_{0}}\left[u_{t}^{(1)}(x) u_{t}^{(2)}(x)\right] \leq\left\|\mathbf{u}_{0}\right\|_{\infty}^{2}\left(1-\frac{\varrho \gamma}{2\left(1-p_{d}\right)}\right)^{-1} .
$$

If $\frac{\varrho \gamma}{2\left(1-p_{d}\right)} \geq 1$, then $\lim _{t \rightarrow \infty} \mathbb{E}_{(\mathbb{1}, \mathbb{1})}\left[u_{t}^{(1)}(x) u_{t}^{(2)}(x)\right]=\infty$.

REMARK 2.7. (a) Note for part (ii) that $\frac{\varrho \gamma}{2\left(1-p_{d}\right)}<1$ for all $\gamma>0$ only if $\varrho \leq 0$. In particular $\varrho=0$ has uniformly bounded second moments in $d \geq 3$.

(b) Results on the second moments of $\operatorname{dSBM}(\varrho, \gamma)$ have already been obtained in [2], Proposition 2.3. We give an alternative proof using the explicit control on $M_{t}^{[\gamma]}$; see the representation (29) below. However, the latter is no longer true for higher moments and instead $M_{t}^{[\gamma]}$ is a random product of matrices [namely the matrices $K_{\tau_{k+1}-\tau_{k}}\left(\cdot, \pi\left(X_{\tau_{k}}\right)\right)$ in the terminology of Section 4]. Since the leading eigenvalue and corresponding eigenvectors are known explicitly, there is some hope to obtain results. Nevertheless, since the random matrices are neither independent nor stationary, this is not trivial.

2.2. The case $\varrho=-1$. In Theorem 2.5 , we excluded $\varrho=-1$ since in this case the infinite rate limit has not yet been constructed for general initial conditions. Our third main result remedies this situation and establishes the infinite 
rate symbiotic branching model $\operatorname{SBM}(-1, \infty)$ for both continuous and discrete space, and characterizes it via the moment duality (6) and the Markov property. In order to state the result properly, we introduce the following additional notation: For $\mathcal{S} \in\left\{\mathbb{Z}^{d}, \mathbb{R}\right\}$, consider the space of all (equivalence classes of) nonnegative bounded measurable functions on $\mathcal{S}$, which obviously can be identified with the space of all Radon measures on $\mathcal{S}$ having bounded densities w.r.t. Lebesgue measure if $\mathcal{S}=\mathbb{R}$ resp. counting measure if $\mathcal{S}=\mathbb{Z}^{d}$. Writing $\mathcal{M}_{b}(\mathcal{S})$ for this space, we have $\mathcal{M}_{b}(\mathcal{S}) \subseteq \mathcal{M}_{\text {tem }}(\mathcal{S})$, and we topologize $\mathcal{M}_{b}(\mathcal{S})$ by the subspace topology inherited from $\mathcal{M}_{\text {tem }}(\mathcal{S})$. Moreover, for each $K>0$ we write $\mathcal{M}_{K}(\mathcal{S})$ for the space of all pairs $\mathbf{u}=\left(u^{(1)}, u^{(2)}\right) \in \mathcal{M}_{b}(\mathcal{S})^{2}$ such that the sum of the densities $u^{(1)}+u^{(2)}$ is bounded by the constant $K$. Observe that $\bigcup_{K>0} \mathcal{M}_{K}(\mathcal{S})=$ $\mathcal{M}_{b}(\mathcal{S})^{2} \subseteq \mathcal{M}_{\text {tem }}(\mathcal{S})^{2}$, and again we endow each $\mathcal{M}_{K}(\mathcal{S})$ with the subspace topology inherited from $\mathcal{M}_{\text {tem }}(\mathcal{S})^{2}$. It is easy to see that convergence in $\mathcal{M}_{K}(\mathcal{S})$ w.r.t. this topology coincides with vague convergence of (pairs of) measures, and that $\mathcal{M}_{K}(\mathcal{S})$ is a compact space. Finally, we denote by $\mathcal{M}_{K}^{\text {sep }}(\mathcal{S}) \subseteq \mathcal{M}_{K}(\mathcal{S})$ the subspace of all $\mathbf{u}=\left(u^{(1)}, u^{(2)}\right) \in \mathcal{M}_{K}(\mathcal{S})$ such that the measures $u^{(1)}$ and $u^{(2)}$ are mutually singular.

THEOREM 2.8. Let $\varrho=-1$. For $\mathcal{S} \in\left\{\mathbb{Z}^{d}, \mathbb{R}\right\}, K>0$ and initial condition $\mathbf{u}_{0} \in \mathcal{M}_{K}(\mathcal{S})$, let $\left(\mathbf{u}_{t}^{[\gamma]}\right)_{t \geq 0}$ denote the finite rate symbiotic branching process $\operatorname{SBM}(-1, \gamma)_{\mathbf{u}_{0}}$, considered as $\mathcal{M}_{K}(\mathcal{S})$-valued process.

(a) (Continuous case) Suppose $\mathcal{S}=\mathbb{R}$. There exists a unique Feller semigroup $^{3}\left(P_{t}\right)_{t \geq 0}$ on $\mathcal{M}_{K}(\mathbb{R})$ such that the corresponding Markov Feller process $\left(\mathbf{u}_{t}\right)_{t \geq 0}$ is characterized by the moment duality (6) for all $n \in \mathbb{N}$. For each initial condition $\mathbf{u}_{0} \in \mathcal{M}_{K}(\mathbb{R})$, the process $\left(\mathbf{u}_{t}\right)_{t \geq 0}$ has continuous sample paths and satisfies the separation of types-property at fixed positive times, that is, for all $t>0$ we have, almost surely, $\mathbf{u}_{t} \in \mathcal{M}_{K}^{\mathrm{sep}}(\mathbb{R})$. Moreover, we have the weak convergence

$$
\mathcal{L}\left(\left(\mathbf{u}_{t}^{[\gamma]}\right)_{t \geq 0} \mid \mathbb{P}_{\mathbf{u}_{0}}\right) \rightarrow \mathcal{L}\left(\left(\mathbf{u}_{t}\right)_{t \geq 0} \mid \mathbb{P}_{\mathbf{u}_{0}}\right)
$$

as $\gamma \rightarrow \infty$ in $\mathcal{C}_{[0, \infty)}\left(\mathcal{M}_{K}(\mathbb{R})\right)$ w.r.t. the uniform topology on compacts.

(b) (Discrete case) Suppose $\mathcal{S}=\mathbb{Z}^{d}$. There exists a unique Feller semigroup $\left(P_{t}\right)_{t \geq 0}$ on $\mathcal{M}_{K}^{\mathrm{sep}}\left(\mathbb{Z}^{d}\right)$ such that the corresponding Markov Feller process $\left(\mathbf{u}_{t}\right)_{t \geq 0} \in$ $D_{[0, \infty)}\left(\mathcal{M}_{K}^{\mathrm{sep}}\left(\mathbb{Z}^{d}\right)\right)$ is characterized by the moment duality (6) for all $n \in \mathbb{N}$. Moreover, denoting by $\mathcal{P}\left(\mathcal{M}_{K}^{\mathrm{sep}}\left(\mathbb{Z}^{d}\right)\right)$ the space of probability measures on $\mathcal{M}_{K}^{\mathrm{sep}}\left(\mathbb{Z}^{d}\right)$, there exists an injection $J: \mathcal{M}_{K}\left(\mathbb{Z}^{d}\right) \rightarrow \mathcal{P}\left(\mathcal{M}_{K}^{\text {sep }}\left(\mathbb{Z}^{d}\right)\right)$ with $J\left(\mathbf{u}_{0}\right)=\delta_{\mathbf{u}_{0}}(\cdot)$ for all $\mathbf{u}_{0} \in \mathcal{M}_{K}^{\mathrm{sep}}\left(\mathbb{Z}^{d}\right)$ such that for all $\mathbf{u}_{0} \in \mathcal{M}_{K}\left(\mathbb{Z}^{d}\right)$ we have the weak convergence

$$
\mathcal{L}\left(\left(\mathbf{u}_{t}^{[\gamma]}\right)_{t \geq 0} \mid \mathbb{P}_{\mathbf{u}_{0}}\right) \rightarrow \mathcal{L}\left(\left(\mathbf{u}_{t}\right)_{t \geq 0} \mid \mathbb{P}_{J\left(\mathbf{u}_{0}\right)}\right)
$$

\footnotetext{
${ }^{3}$ For a compact space $E$, by a Feller semigroup on $E$ we mean a Markov semigroup $\left(P_{t}\right)_{t \geq 0}$ which satisfies $P_{t}(\mathcal{C}(E)) \subset \mathcal{C}(E)$ and is strongly continuous on $\mathcal{C}(E)$.
} 
as $\gamma \rightarrow \infty$ in $D_{[0, \infty)}\left(\mathcal{M}_{K}\left(\mathbb{Z}^{d}\right)\right)$ w.r.t. the Meyer-Zheng topology. Defining $w_{0}:=$ $u_{0}^{(1)}+u_{0}^{(2)}$, the function $J$ is explicitly given by

$$
J\left(\mathbf{u}_{0}\right)=\bigotimes_{x \in \mathbb{Z}^{d}}\left[\left(\frac{u_{0}^{(1)}(x)}{w_{0}(x)} \delta_{\left(w_{0}(x), 0\right)}+\frac{u_{0}^{(2)}(x)}{w_{0}(x)} \delta_{\left(0, w_{0}(x)\right)}\right) \mathbb{1}_{\left\{w_{0}(x)>0\right\}}\right.
$$

$$
\left.+\delta_{(0,0)} \mathbb{1}_{\left\{w_{0}(x)=0\right\}}\right] \text {. }
$$

REMARK 2.9. We will refer to the process $\left(\mathbf{u}_{t}\right)_{t \geq 0}$ in Theorem 2.8 as the infinite rate symbiotic branching process $\operatorname{SBM}(-1, \infty)[\operatorname{resp} \cdot \operatorname{cSBM}(-1, \infty)$ for $\mathcal{S}=\mathbb{R}$ and $\mathrm{dSBM}(-1, \infty)$ for $\left.\mathcal{S}=\mathbb{Z}^{d}\right]$. For continuous space, Theorem 2.8(a) extends the results of [4] to the case $\varrho=-1$, which was left open in that paper. The characterization of the limit in [4], Theorems 1.10,1.12, in terms of a martingale problem is replaced by a characterization via moments. The latter is possible, since for $\varrho=-1$ the noises are perfectly negatively correlated so that the sum of the solutions solves the heat equation, thus the solutions are dominated by a deterministic function.

We remark that for the continuous-space stepping stone case (1) where $u_{0}^{(1)}+$ $u_{0}^{(2)}=1$, the result in part (a) (except the convergence assertion, and with a different dual process) can be considered a two-type version of the results proved in [12], Theorem 4.1, Proposition 5.1, and [7], Corollary 7.3. In an infinitely-manytypes setting, these authors consider also more general migration processes than Brownian motion and define the "limiting" process directly from the duality. On the other hand, in discrete space the stepping stone model is well known to converge (in the Meyer-Zheng and f.d.d. sense) to the voter model as $\gamma \rightarrow \infty$; see, for example, [8], Theorem 4.16, thus in part (b) we cannot expect continuous paths of the limiting process and convergence in the Skorokhod topology.

The fact that for $\mathcal{S}=\mathbb{R}$ in part (a) we obtain continuity of the paths at $t=0$ may seem counterintuitive, since the separation of types-property holds for positive times but we allow for arbitrary (not necessarily separated) initial conditions. The reason is that in the continuous case the topology on $\mathcal{M}_{K}(\mathbb{R})$ is so weak that the separation of types is not preserved under convergence of the measures, that is, $\mathcal{M}_{K}^{\text {sep }}(\mathbb{R})$ is not closed in $\mathcal{M}_{K}(\mathbb{R})$ (on the contrary, it is a dense subset). This is a major difference to the discrete case, where the separation of types is preserved under convergence in $\mathcal{M}_{K}\left(\mathbb{Z}^{d}\right)$. Thus, in part (b), for the definition of $\operatorname{dSBM}(-1, \infty)$ and the corresponding semigroup we restrict to initial conditions $\mathbf{u}_{0} \in \mathcal{M}_{K}^{\mathrm{sep}}\left(\mathbb{Z}^{d}\right)$, in agreement with the case $\varrho>-1$.

We now proceed to a more explicit characterization of the limit in Theorem 2.8 for the continuous-space case. First, we collect some additional notation: Let $\mathcal{U}$ denote the space of all pairs of absolutely continuous Radon measures $\mathbf{u}=\left(u^{(1)}, u^{(2)}\right)$ on $\mathbb{R}$ with bounded densities such that $u^{(1)}$ and $u^{(2)}$ are 
mutually singular and $u^{(1)}+u^{(2)}$ is equivalent to Lebesgue measure [equivalently, $u^{(1)}(x) u^{(2)}(x)=0$ and $u^{(1)}(x)+u^{(2)}(x)>0$ for almost all $\left.x \in \mathbb{R}\right]$. Note that $\mathcal{U} \subseteq \bigcup_{K>0} \mathcal{M}_{K}^{\text {sep }}(\mathbb{R}) \subseteq \mathcal{M}_{b}(\mathbb{R})^{2} \subseteq \mathcal{M}_{\text {tem }}(\mathbb{R})^{2}$, and again we topologize $\mathcal{U}$ with the subspace topology inherited from $\mathcal{M}_{\text {tem }}(\mathbb{R})^{2}$. For $\mathbf{u} \in \mathcal{U}$, we define

$$
\mathcal{I}(\mathbf{u}):=\operatorname{supp}\left(u^{(1)}\right) \cap \operatorname{supp}\left(u^{(2)}\right),
$$

where $\operatorname{supp}(u)$ denotes the measure-theoretic support of $u \in \mathcal{U}$, that is,

$$
\operatorname{supp}(u):=\left\{x \in \mathbb{R}: u\left(B_{\varepsilon}(x)\right)>0 \text { for all } \varepsilon>0\right\} .
$$

We call the elements of $\mathcal{I}(\mathbf{u})$ interface points or just interfaces. The configurations with exactly $n \in \mathbb{N}$ interface points are denoted by $\mathcal{U}_{n}$, that is,

$$
\mathcal{U}_{n}:=\{\mathbf{u} \in \mathcal{U}:|\mathcal{I}(\mathbf{u})|=n\} .
$$

We write $m(\mathbf{u}, x):=1$ if $x \in \operatorname{supp}\left(u^{(1)}\right) \backslash \mathcal{I}(u)$ and $m(\mathbf{u}, x):=2$ if $x \in \operatorname{supp}\left(u^{(2)}\right) \backslash$ $\mathcal{I}(u)$, while setting $m(\mathbf{u}, x):=0$ if $x \in \mathcal{I}(u)$.

Throughout the rest of this section, given initial conditions $\mathbf{u}_{0}=\left(u_{0}^{(1)}, u_{0}^{(2)}\right) \in$ $\mathcal{M}_{b}(\mathbb{R})^{2}$ for $\operatorname{cSBM}(-1, \infty)_{\mathbf{u}_{0}}$, we write

$$
w_{t}:=S_{t} w_{0}
$$

for the solution to the deterministic heat equation with initial condition $w_{0}:=$ $u_{0}^{(1)}+u_{0}^{(2)}$ and recall that since $\varrho=-1$, we know $u_{t}^{(1)}+u_{t}^{(2)}=w_{t}$ for all $t \geq 0$. Note that in view of Theorem 2.8(a), for each fixed $t>0$ we have almost surely $\mathbf{u}_{t} \in \mathcal{U}$, provided $w_{0} \neq 0$.

Our next result deals with initial conditions of "single interface type".

THEOREM 2.10. Assume $\mathbf{u}_{0} \in \mathcal{U}_{1}$. Let $\left(\mathbf{u}_{t}\right)_{t \geq 0}$ denote the solution of $\operatorname{cSBM}(-1, \infty)_{\mathbf{u}_{0}}$. Then we have, almost surely,

$$
\mathbf{u}_{t} \in \mathcal{U}_{1} \quad \text { for all } t \geq 0 .
$$

Let $\left(I_{t}\right)_{t \geq 0}$ denote the single interface process defined by the unique element of $\mathcal{I}\left(\mathbf{u}_{t}\right), t>0$. Then, almost surely $\left(I_{t}\right)_{t \geq 0}$ is continuous and there exists a standard Brownian motion $\left(B_{t}\right)_{t \geq 0}$ such that

$$
I_{t}=I_{0}-\int_{0}^{t} \frac{w_{s}^{\prime}\left(I_{s}\right)}{w_{s}\left(I_{s}\right)} d s+B_{t}, \quad t \geq 0,
$$

and the process $\left(I_{t}\right)_{t \geq 0}$ is the unique (in law) weak solution of the SDE (9). ${ }^{4}$ Moreover, $\mathbf{u}_{t}$ can be recovered, for $i=1,2$, as

$$
u_{t}^{(i)}(d x)= \begin{cases}\mathbb{1}_{\left\{x \leq I_{t}\right\}} w_{t}(x) d x & \text { if } \lim _{x \rightarrow-\infty} m\left(\mathbf{u}_{0}, x\right)=i, \\ \mathbb{1}_{\left\{x \geq I_{t}\right\}} w_{t}(x) d x & \text { if } \lim _{x \rightarrow-\infty} m\left(\mathbf{u}_{0}, x\right)=3-i .\end{cases}
$$

\footnotetext{
${ }^{4}$ Under the assumption $\mathbf{u}_{0} \in \mathcal{U}_{1}$, the integrand on the right hand side of (9) is not guaranteed to be Lebesgue-integrable at 0 . However, in any case the integral exists as an improper integral $\lim _{\varepsilon \downarrow 0} \int_{\varepsilon}^{t} \frac{w_{s}^{\prime}\left(I_{s}\right)}{w_{s}\left(I_{s}\right)} d s$.
} 
REMARK 2.11. If $\mathbf{u}_{0} \in \mathcal{U}_{1}$ is such that $w_{0}:=u_{0}^{(1)}+u_{0}^{(2)}$ is continuously differentiable with $\sup _{x \in \mathbb{R}}\left|w_{0}^{\prime}(x)\right|<\infty$ and $\inf _{x \in \mathbb{R}} w_{0}(x)>0$, then the drift term $(s, x) \mapsto \frac{w_{s}^{\prime}(x)}{w_{s}(x)}$ in equation (9) is continuous and globally bounded on $\mathbb{R}^{+} \times \mathbb{R}$, and existence and uniqueness of a weak solution to the SDE follow from the standard theory (see e.g. [23] or [16], Chapter 5.3). In particular, in this case the integrand in (9) is bounded and the integral is a proper integral. For general $\mathbf{u}_{0} \in \mathcal{U}_{1}$ however, we know only that $w_{0}$ is bounded and strictly positive almost everywhere, and standard theory does not cover existence and uniqueness for equation (9). In particular, it can occur that $w_{0}\left(I_{0}\right)=0, w_{0}^{\prime}\left(I_{0}-\right)=-\infty$ and $w_{0}^{\prime}\left(I_{0}+\right)=+\infty$ so that the integrand need not even be locally bounded. Our result shows that nevertheless the SDE (9) has a unique weak solution.

Our next result covers the case that $\mathcal{I}\left(\mathbf{u}_{0}\right)$ consists of more than a single pointeven infinitely many points - as long as there are no accumulation points. Informally, in this case the interfaces follow each the dynamics of a Brownian motion with drift as in (9) and upon collision both motions annihilate.

To describe the limiting system formally, we introduce the following terminology: We call a collection $\left\{\left(Y_{t}^{i}\right)_{t \geq 0}: i \in J\right\}$ of càdlàg stochastic processes indexed by an at most countable set $J$ and taking values in $\mathbb{R} \cup\{\dagger\}$, where $\dagger$ is interpreted as a cemetery state, a regular annihilating system if, almost surely, the following hold:

- The initial positions are distinct and $\left\{Y_{0}^{i}\right\}_{i \in J}$ has no accumulation points.

- $Y_{t}^{i}=\dagger$ if and only if there exist $s \leq t$ and $j \in J \backslash\{i\}$ such that $Y_{s-}^{i}=Y_{s-}^{j} \in \mathbb{R}$.

- $\tau_{i}:=\inf \left\{t \geq 0: Y_{t}^{i}=\dagger\right\}>0$.

- Each process $Y^{i}$ is continuous at any time $t<\tau_{i}$.

- There are no triple annihilations, that is, there are no times $t$ such that $Y_{t-}^{i}=$ $Y_{t-}^{j}=Y_{t-}^{k} \in \mathbb{R}$ for distinct $i, j, k \in J$.

Now suppose we are given $\mathbf{u}_{0} \in \mathcal{U}$ such that $\mathcal{I}\left(\mathbf{u}_{0}\right)$ has no accumulation points and a regular annihilating system $\left\{\left(Y_{t}^{x}\right)_{t \geq 0}: x \in \mathcal{I}\left(\mathbf{u}_{0}\right)\right\}$ indexed by and starting from $\mathcal{I}\left(\mathbf{u}_{0}\right)$. Consider the partition of $[0, \infty) \times \mathbb{R}$ induced by the graphs of the annihilating paths: We can "color" each component of the partition in a way that is consistent with the coloring $m\left(\mathbf{u}_{0}, \cdot\right)$ of $\mathbb{R}$. More precisely, define a mapping $\hat{m}:[0, \infty) \times \mathbb{R} \rightarrow\{0,1,2\}$ as follows: Let $\hat{m}$ be equal to 0 on the closure of the graphs of the annihilating paths, that is,

$$
\hat{m}(t, x):=0 \quad \text { for }(t, x) \in \mathcal{J}:=\operatorname{cl}\left(\bigcup_{j \in J}\left\{\left(t, Y_{t}^{j}\right): t \in\left[0, \tau_{j}\right)\right\}\right) .
$$

Then setting

$$
\hat{m}(0, x):=m\left(\mathbf{u}_{0}, x\right) \quad \text { for } x \in \mathbb{R}
$$




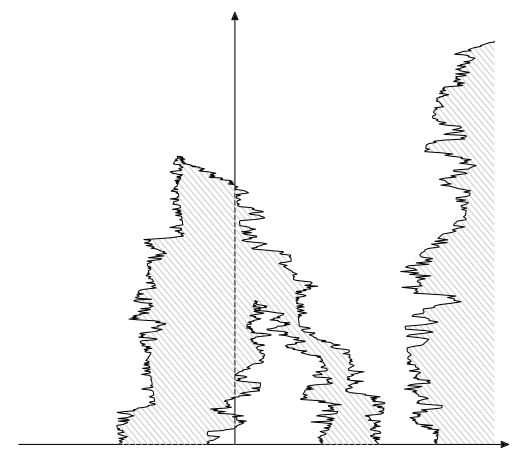

FIG. 1. An illustration of the standard coloring $\hat{m}$ of $[0, \infty) \times \mathbb{R}$ induced by an initial configuration $\mathbf{u}_{0}$ with five interfaces and a regular annihilating system starting from $\mathcal{I}\left(\mathbf{u}_{0}\right)$. Type 1 is drawn in white and type 2 is shaded grey. The corresponding standard element $\left(\hat{\mathbf{u}}_{t}\right)_{t \geq 0} \in \mathcal{C}_{[0, \infty)}(\mathcal{U})$ is such that $\hat{\mathbf{u}}_{t}^{(1)}(x)\left[\right.$ resp. $\left.\hat{\mathbf{u}}_{t}^{(2)}(x)\right]$ agrees with $w_{t}(x)$ for all $(t, x)$ in the white (resp. shaded) area and is zero otherwise.

$\hat{m}(\cdot, \cdot)$ is defined by the requirement that it is locally constant on the complement $\mathcal{J}^{c}$, cf. Figure 1 . We call $\hat{m}$ the standard coloring of $[0, \infty) \times \mathbb{R}$ induced by $\mathbf{u}_{0}$ and $\left\{\left(Y_{t}^{x}\right)_{t \geq 0}: x \in \mathcal{I}\left(\mathbf{u}_{0}\right)\right\}$. Obviously, $\hat{m}(t, x)$ is the unique extension of $\hat{m}(0, x)=$ $m\left(\mathbf{u}_{0}, x\right)$ from $\{0\} \times \mathbb{R}$ to $[0, \infty) \times \mathbb{R}$ which is continuous on $\mathcal{J}^{c}$, jointly in the variables $(t, x)$.

Using $\hat{m}$, we define

$$
\hat{\mathbf{u}}_{t}(x):=\left(w_{t}(x) \mathbb{1}_{\{\hat{m}(t, x)=1\}}, w_{t}(x) \mathbb{1}_{\{\hat{m}(t, x)=2\}}\right), \quad t \geq 0, x \in \mathbb{R} .
$$

It is easy to see that $\hat{\mathbf{u}}_{t} \in \mathcal{U}$ for each $t>0$ and (by the properties of the regular annihilating system) that the process $\left(\hat{\mathbf{u}}_{t}\right)_{t \geq 0}$ has continuous paths [recall that $\mathcal{U}$ is topologized by the subspace topology inherited from $\left.\mathcal{M}_{\text {tem }}(\mathbb{R})^{2}\right]$. We call $\left(\hat{\mathbf{u}}_{t}\right)_{t \geq 0}$ the standard element of $\mathcal{C}_{[0, \infty)}(\mathcal{U})$ induced by $\mathbf{u}_{0}$ and $\left\{\left(Y_{t}^{x}\right)_{t \geq 0}: x \in \mathcal{I}\left(\mathbf{u}_{0}\right)\right\}$.

THEOREM 2.12. Assume that $\mathbf{u}_{0} \in \mathcal{U}$ and $\mathcal{I}\left(\mathbf{u}_{0}\right)$ has no accumulation points. Let $\left(\mathbf{u}_{t}\right)_{t \geq 0}$ denote the infinite rate limit $\left.\mathrm{cSBM}(-1, \infty)\right)_{\mathbf{u}_{0}}$. Then there exists a regular annihilating system $\left\{\left(I_{t}^{x}\right)_{t \geq 0}: x \in \mathcal{I}\left(\mathbf{u}_{0}\right)\right\}$ starting from $\mathcal{I}\left(\mathbf{u}_{0}\right)$ such that each coordinate independently follows the law of the single-point interface process of Theorem 2.10 up to the first collision with another (surviving) motion, upon which both motions annihilate. Denote by $\left(\hat{\mathbf{u}}_{t}\right)_{t \geq 0}$ the standard element of $\mathcal{C}_{[0, \infty)}(\mathcal{U})$ induced by $\mathbf{u}_{0}$ and $\left\{\left(I_{t}^{x}\right)_{t \geq 0}: x \in \mathcal{I}\left(\mathbf{u}_{0}\right)\right\}$, as defined in (10). Then we have

$$
\left(\mathbf{u}_{t}\right)_{t \geq 0} \stackrel{d}{=}\left(\hat{\mathbf{u}}_{t}\right)_{t \geq 0} \quad \text { on } \mathcal{C}_{[0, \infty)}\left(\mathcal{M}_{b}(\mathbb{R})^{2}\right) .
$$

REMARK 2.13. If $\mathcal{I}\left(\mathbf{u}_{0}\right)$ is finite, then it is obvious how to construct the regular annihilating system. However, due to the lack of monotonicity, it becomes trickier to define the system for infinitely many particles. Our construction uses a 
coupling which embeds the annihilating system into a system of instantaneously coalescing Brownian motions with drift, as also used (in a random walks context) by [1].

We now deal with the case of completely general initial densities, which do not have to be mutually singular anymore. Here the situation is more involved, as the set of interface points $\mathcal{I}\left(\mathbf{u}_{0}\right)$ can be an interval or even $\mathbb{R}$. Although we know already by Theorem 2.8 (a) that the measures $u_{t}^{(1)}$ and $u_{t}^{(2)}$ are mutually singular at each positive time, a priori the set $\mathcal{I}\left(\mathbf{u}_{t}\right)$ might still be very complicated. Our final result states that for all $t>0$ the set of interface points is in fact discrete, and these points move as in the case of Theorem 2.12.

THEOREM 2.14. Assume initial densities $\mathbf{u}_{0}=\left(u_{0}^{(1)}, u_{0}^{(2)}\right) \in \mathcal{M}_{b}(\mathbb{R})^{2}$ such that $w_{0}:=u_{0}^{(1)}+u_{0}^{(2)} \neq 0$. Let $\left(\mathbf{u}_{t}\right)_{t \geq 0}$ denote the infinite rate limit $\operatorname{cSBM}(-1$, $\infty)_{\mathbf{u}_{0}}$. Then, almost surely, $\mathcal{I}\left(\mathbf{u}_{t}\right)$ contains no accumulation point for any $t>0$. Moreover, for any $t_{0}>0$, the law of $\left(\mathbf{u}_{t}\right)_{t \geq t_{0}}$ is given as in Theorem 2.12 when started in $\mathbf{u}_{t_{0}}$.

REMARK 2.15. (a) In [24], Tribe considered the special case of complementary initial densities $\mathbf{u}_{0}$ where the types are separated by finitely many interfaces and $u_{0}^{(1)}+u_{0}^{(2)} \equiv 1$. In that case, Tribe proved that the interfaces move as annihilating Brownian motions (without drift).

In contrast, we allow for essentially arbitrary initial conditions. If $u_{0}^{(1)}+u_{0}^{(2)} \neq$ 1 , the dynamics of interfaces is influenced by the relative "height" difference of the two populations at either side of the interfaces, yielding the additional drift term in (9). Moreover, we require neither a finite number of interfaces nor even the initial separation of types. As Theorem 2.14 shows, the process "comes down from infinity" in the sense that for any positive time, locally there are only finitely many interfaces.

(b) Entrance laws for annihilating Brownian motions. For $u_{0}^{(1)}+u_{0}^{(2)} \equiv 1$, Theorem 2.14 allows us to characterize entrance laws for annihilating Brownian motions. As in [25], Section 2.3, a particularly interesting entrance law can be obtained by approximation from a system of annihilating Brownian motions starting at the points of a Poisson point process with intensity $\lambda$ and letting $\lambda \rightarrow \infty$. Informally, this entrance law corresponds to a system of annihilating Brownian motions "starting from every point" on the real line. However, as already observed in [25], the examples in [5], Section 3, suggest that other approximations may lead to different entrance laws. By using Theorem 2.14 and the relation to $\operatorname{cSBM}(-1, \infty)$, one can clearly see why those examples can lead to different entrance laws, and in fact we can obtain a complete classification of entrance laws for annihilating Brownian motions. For details of this correspondence, we refer to [14]. 
The remaining paper is structured as follows: We start by showing in Section 3 how to reinterpret the original moment duality and we prove Theorem 2.1. Then, in Section 4 we can send $\gamma \rightarrow \infty$ in the reinterpretation of the duality and we prove Theorems 2.3, 2.5 and Corollary 2.6. In Section 5, we specialize to the case $\varrho=-1$, establishing first the existence, uniqueness and basic properties of $\operatorname{SBM}(-1, \infty)$ by proving Theorem 2.8 . We then proceed to the explicit description of the limiting process and prove Theorems 2.10, 2.12 and 2.14.

3. The reinterpretation of the moment duality. In this section, we prove Theorem 2.1. The proof is based on the original moment duality (2). In this entire section we assume $\gamma>0, \varrho \in[-1,1]$ and $n \in \mathbb{N}$ to be fixed.

For $\widetilde{M}_{0} \in \mathcal{M}\left(\{1,2\}^{n}\right)$, define for all $b \in\{1,2\}^{n}$,

$$
\widetilde{M}_{t}\left(X, \widetilde{M}_{0}\right)(b):=\sum_{c \in\{1,2\}^{n}} \widetilde{M}_{0}(c) \mathbb{E}_{c}\left[e^{\gamma\left(L_{t}^{=}+\varrho L_{t}^{\neq}\right)} \mathbb{1}_{C_{t}=b} \mid X_{[0, t]}\right],
$$

that is, $\widetilde{M}_{t}(\cdot, c) \in \mathcal{M}\left(\{1,2\}^{n}\right)$ is the expected exponential correction term as a function of the paths $X=\left(X_{t}^{(1)}, \ldots, X_{t}^{(n)}\right)_{t \geq 0}$ of either random walks or Brownian motions and interpreted as a measure on the colorings. Note that $\widetilde{M}_{t}\left(X, \widetilde{M}_{0}\right)$ is measurable w.r.t. $\sigma\left(X_{s}: 0 \leq s \leq t\right)$. We will show that $\widetilde{M}_{t}$ coincides with $M_{t}^{[\gamma]}$ from (4). With this, Theorem 2.1 directly follows from the original duality (2).

Lemma 3.1. Fix $s, t \geq 0$. Let $\left(\theta_{t} X\right)_{r}:=X_{t+r}$ be the shift operator. Then

$$
\begin{aligned}
\widetilde{M}_{t+s}\left(X, \widetilde{M}_{0}\right) & =\widetilde{M}_{t+s}\left(X_{[0, t+s]}, \widetilde{M}_{0}\right) \\
& =\widetilde{M}_{s}\left(\left(\theta_{t} X\right)_{[0, s]}, \widetilde{M}_{t}\left(X_{[0, t]}, \widetilde{M}_{0}\right)\right)
\end{aligned}
$$

almost surely with respect to the law of $X$.

Proof. By the definition of $\widetilde{M}_{t+s}$,

$$
\begin{aligned}
\widetilde{M}_{t+s} & \left(X, \widetilde{M}_{0}\right)(b) \\
= & \sum_{c \in\{1,2\}^{n}} \widetilde{M}_{0}(c) \mathbb{E}_{c}\left[e^{\gamma\left(L_{t+s}^{=}+\varrho L_{t+s}^{\neq}\right)} \mathbb{1}_{C_{t+s}=b} \mid X_{[0, t+s]}\right] \\
= & \sum_{c \in\{1,2\}^{n}} \widetilde{M}_{0}(c) \mathbb{E}_{c}\left[e^{\gamma\left(L_{t}^{=}+\varrho L_{t}^{\neq}\right)}\right. \\
& \left.\times \mathbb{E}\left[e^{\gamma\left(L_{t+s}^{\bar{E}}-L_{t}^{\bar{E}}+\varrho\left(L_{t+s}^{\neq}-L_{t}^{\neq}\right)\right)} \mathbb{1}_{C_{t+s}=b} \mid C_{[0, t]}, X_{[0, t+s]}\right] \mid X_{[0, t+s]}\right] .
\end{aligned}
$$

The increments of the local times, $L_{t+s}^{=}-L_{t}^{=}, L_{t+s}^{\neq}-L_{t}^{\neq}$, are a function of $\left(\theta_{t} X, \theta_{t} C\right)_{[0, s]}$ and equal the local times $L_{s}^{=}, L_{s}^{\neq}$of $\theta_{t} X$ and $\theta_{t} C$. Furthermore, conditioned on $X$, the coloring process $\left(C_{t}\right)_{t \geq 0}$ is a time-inhomogeneous Markov 
process, and the law of $C_{t+s}$ depends only on $C_{t}$ and the path $\left(\theta_{t} X\right)_{[0, s]}$. Therefore,

$$
\begin{gathered}
\mathbb{E}\left[e^{\gamma\left(L_{t+s}^{=}-L_{t}^{=}+\varrho\left(L_{t+s}^{\neq}-L_{t}^{\neq}\right)\right)} \mathbb{1}_{C_{t+s}=b} \mid C_{[0, t]}, X_{[0, t+s]}\right] \\
=\mathbb{E}\left[e^{\gamma\left(\theta_{t} L_{s}^{=}+\varrho \theta_{t} L_{s}^{\neq}\right)} \mathbb{1}_{\theta_{t} C_{s}=b} \mid C_{t},\left(\theta_{t} X\right)_{[0, s]}\right] .
\end{gathered}
$$

Summing over all possible values of $C_{t}$ and using again that $C_{t}$ and the local times $L_{t}^{=}, L_{t}^{\neq}$depend only on $X_{[0, t]}$, we get

$$
\begin{aligned}
\widetilde{M}_{t+s}( & \left.X, \widetilde{M}_{0}\right)(b) \\
= & \sum_{c, c^{\prime} \in\{1,2\}^{n}} \widetilde{M}_{0}(c) \mathbb{E}_{c}\left[e^{\gamma\left(L_{t}^{=}+\varrho L_{t}^{\neq}\right)} \mathbb{1}_{C_{t}=c^{\prime}} \mid X_{[0, t]}\right] \\
& \times \mathbb{E}\left[e^{\gamma\left(\theta_{t} L_{s}^{=}+\varrho \theta_{t} L_{s}^{\neq}\right)} \mathbb{1}_{\theta_{t} C_{s}=b} \mid C_{t}=c^{\prime},\left(\theta_{t} X\right)_{[0, s]}\right] \\
= & \sum_{c^{\prime} \in\{1,2\}^{n}} \widetilde{M}_{t}\left(X_{[0, t]}, \widetilde{M}_{0}\right)\left(c^{\prime}\right) \mathbb{E}\left[e^{\gamma\left(\theta_{t} L_{s}^{=}+\varrho \theta_{t} L_{s}^{\neq}\right)} \mathbb{1}_{\theta_{t} C_{s}=b} \mid C_{t}=c^{\prime},\left(\theta_{t} X\right)_{[0, s]}\right] \\
= & \widetilde{M}_{s}\left(\left(\theta_{t} X\right)_{[0, s]}, \widetilde{M}_{t}\left(X_{[0, t]}, \widetilde{M}_{0}\right)\right)(b) .
\end{aligned}
$$

We now have to distinguish between the discrete and the continuous case, since collisions and local times of random walks and Brownian motions behave somewhat differently. In particular, in the discrete case collisions between more than two random walks can happen.

LeMma 3.2. Assume the discrete space case, where $X=\left(X^{(1)}, \ldots, X^{(n)}\right)$ is the collection of $n$ independent random walks on $\mathbb{Z}^{d}$. Let $\Gamma_{t}:=\left\{(i, j): X_{t}^{(i)}=\right.$ $\left.X_{t}^{(j)}, 1 \leq i<j \leq n\right\}$ be the set of collision pairs at time $t$, and for $c \in\{1,2\}^{n}$ let $\Gamma_{t}^{=}(c):=\left\{(i, j) \in \Gamma_{t}: c_{i}=c_{j}\right\}$ and $\Gamma_{t}^{\neq}(c):=\left\{(i, j) \in \Gamma_{t}: c_{i} \neq c_{j}\right\}$ be the decomposition into same color collisions and different color collisions according to the coloring $c$. Then $\widetilde{M}_{t}=\widetilde{M}_{t}\left(X, \widetilde{M}_{0}\right)$ satisfies the following linear ODE:

$$
\frac{d}{d t} \widetilde{M}_{t}(b)=\gamma \varrho\left|\Gamma_{t}^{\neq}(b)\right| \widetilde{M}_{t}(b)+\frac{\gamma}{2} \sum_{\substack{c \in\{1,2\}^{n}: \\ d(b, c)=1}}\left|\Gamma_{t}^{=}(c) \cap \Gamma_{t}^{\neq}(b)\right| \widetilde{M}_{t}(c),
$$

for $b \in\{1,2\}^{n}$, with $d(\cdot, \cdot)$ denoting the Hamming distance on $\{1,2\}^{n}$.

Proof. Since $X$ is right continuous, choose $\epsilon$ small enough that $X_{[t, t+\epsilon]} \equiv$ $X_{t}$. Then by Lemma 3.1

$$
\begin{aligned}
& \widetilde{M}_{t+\epsilon}(b)-\widetilde{M}_{t}(b) \\
& \quad=\sum_{c \in\{1,2\}^{n}} \widetilde{M}_{t}(c) \mathbb{E}\left[e^{\gamma\left(\theta_{t} L_{\epsilon}^{=}+\varrho \theta_{t} L_{\epsilon}^{\neq}\right)} \mathbb{1}_{\theta_{t} C_{\epsilon}=b} \mid C_{t}=c, \theta_{t} X\right]-\widetilde{M}_{t}(b) .
\end{aligned}
$$


Since the total rate of color change in a coloring $c$ is given by $\gamma\left|\Gamma_{t}^{=}(c)\right|<\gamma n^{2}$, the probability of two color changes in time $\epsilon$ is small and the above is equal to

$$
\begin{aligned}
\widetilde{M}_{t}(b) & \left(\mathbb{E}\left[e^{\gamma\left(\theta_{t} L_{\epsilon}^{=}+\varrho \theta_{t} L_{\epsilon}^{\neq}\right)} \mathbb{1}_{\theta_{t} C_{\epsilon}=b} \mid C_{t}=b, \theta_{t} X\right]-1\right) \\
+ & \sum_{c: d(c, b)=1} \widetilde{M}_{t}(c) \mathbb{E}\left[e^{\gamma\left(\theta_{t} L_{\epsilon}^{=}+\varrho \theta_{t} L_{\epsilon}^{\neq}\right)} \mathbb{1}_{\theta_{t} C_{\epsilon}=b} \mid C_{t}=c, \theta_{t} X\right]+O\left(\epsilon^{2}\right) \\
= & \widetilde{M}_{t}(b)\left(e^{\gamma\left(\left|\Gamma_{t}^{=}(b)\right| \epsilon+\varrho\left|\Gamma_{t}^{\neq}(b)\right| \epsilon\right)} e^{-\gamma\left|\Gamma_{t}^{=}(b)\right| \epsilon}-1\right) \\
& +\sum_{c: d(c, b)=1} \widetilde{M}_{t}(c) e^{O(\epsilon)}\left(1-e^{-\frac{\gamma}{2}\left|\Gamma_{t}^{=}(c) \cap \Gamma_{t}^{\neq}(b)\right| \epsilon}\right)+O\left(\epsilon^{2}\right) .
\end{aligned}
$$

In the last line we used the fact that if $d(c, b)=1$, there is one coordinate, say $i$, which changed color. The rate that this color change happens is $\gamma / 2$ times the number of other particles at $X_{t}^{(i)}$ which have color $c_{i}$, and this number is exactly given by pairs of particles which have the same color before the change, and a different afterwards. The exact value of $\gamma\left(L_{\epsilon}^{=}+\varrho L_{\epsilon}^{\neq}\right)$depends on the time of the color change, but is of order $\epsilon$ since its absolute value is bounded by $n^{2} \gamma \epsilon$. By writing $e^{x}=1+x+O\left(x^{2}\right)$, we get that the above is

$$
\widetilde{M}_{t}(b) \varrho \gamma\left|\Gamma_{t}^{\neq}(b)\right| \epsilon+\frac{\gamma}{2} \sum_{c: d(c, b)=1} \widetilde{M}_{t}(c)\left|\Gamma_{t}^{=}(c) \cap \Gamma_{t}^{\neq}(b)\right| \epsilon+O\left(\epsilon^{2}\right) .
$$

Dividing by $\epsilon$ and then sending $\epsilon$ to 0 completes the proof.

LEMMA 3.3 (The discrete case). In the discrete case, we have

$$
\begin{aligned}
\frac{d}{d t} \widetilde{M}_{t}(b)= & \frac{\gamma \varrho}{2} \sum_{i, j=1}^{n} \mathbb{1}_{b_{i} \neq b_{j}} \widetilde{M}_{t}(b) \frac{d L_{t}^{i, j}}{d t} \\
& +\frac{\gamma}{2} \sum_{i, j=1}^{n} \mathbb{1}_{b_{i} \neq b_{j}} \widetilde{M}_{t}\left(\widehat{b}^{i}\right) \frac{d L_{t}^{i, j}}{d t}
\end{aligned}
$$

for $b \in\{1,2\}^{n}$. Here $L_{t}^{i, j}=\int_{0}^{t} \mathbb{1}_{X_{s}^{(i)}=X_{s}^{(j)}} d s$ are the pair local times and $\widehat{b}^{i}$ is the coloring $b$ flipped at $i$.

PROOF. By simple counting we get that $\left|\Gamma_{t}^{\neq}(b)\right|=\frac{1}{2} \sum_{i, j=1}^{n} \mathbb{1}_{b_{i} \neq b_{j}} \mathbb{1}_{X_{t}^{(i)}=X_{t}^{(j)}}$. For each $c$ with $d(c, b)=1$ there is some index $i$ so that $c=\widehat{b}^{i}$. Then

$$
\left|\Gamma_{t}^{=}(c) \cap \Gamma_{t}^{\neq}(b)\right|=\sum_{j=1}^{n} \mathbb{1}_{b_{j} \neq b_{i}} \mathbb{1}_{X_{t}^{(i)}=X_{t}^{(j)}}
$$


and summing over all possible $i$ we get

$$
\sum_{c: d(c, b)=1}\left|\Gamma_{t}^{=}(c) \cap \Gamma_{t}^{\neq}(b)\right|=\sum_{i, j=1}^{n} \mathbb{1}_{b_{i} \neq b_{j}} \mathbb{1}_{X_{t}^{(i)}=X_{t}^{(j)}} .
$$

Now (11) follows from Lemma 3.2.

Note that since (11) and (4) are the same equations, $\widetilde{M}_{t}\left(X, \delta_{c}\right)$ and $M_{t}^{[\gamma]}\left(X, \delta_{c}\right)$ agree, thus Theorem 2.1 is now proven for the discrete case.

The proof for the continuous case follows along the same lines. It is in some parts simpler, as simultaneous collisions between more than two Brownian motions do not happen almost surely. Theorem 2.1 for the continuous case follows from the following lemma, which is a version of Lemma 3.3.

LEMMA 3.4 (The continuous case). Assume the continuous space setting, where $X=\left(X^{(1)}, \ldots, X^{(n)}\right)$ is the collection of $n$ independent Brownian motions. Then $\widetilde{M}_{t}=\widetilde{M}_{t}\left(X, \widetilde{M}_{0}\right)$ satisfies the following linear ODE almost surely w.r.t. the law of $X:$ for $b \in\{1,2\}^{n}$,

$$
d \widetilde{M}_{t}(b)=\frac{\gamma \varrho}{2} \sum_{i, j=1}^{n} \mathbb{1}_{b_{i} \neq b_{j}} \widetilde{M}_{t}(b) d L_{t}^{i, j}+\frac{\gamma}{2} \sum_{i, j=1}^{n} \mathbb{1}_{b_{i} \neq b_{j}} \widetilde{M}_{t}\left(\widehat{b}^{i}\right) d L_{t}^{i, j} .
$$

Proof. Since $X$ is right continuous and almost surely has no multiple collisions, choose $\epsilon>0$ small enough so that $L_{t+\epsilon}^{i, j}=L_{t}^{i, j}$ for all pairs $(i, j)$ except for possibly one, say $(k, \ell)$, where $\kappa:=L_{t+\epsilon}^{k, \ell}-L_{t}^{k, \ell} \geq 0$. Then by Lemma 3.1

$$
\begin{aligned}
& \widetilde{M}_{t+\epsilon}(b)-\widetilde{M}_{t}(b) \\
& \quad=\sum_{c \in\{1,2\}^{n}} \widetilde{M}_{t}(c) \mathbb{E}\left[e^{\gamma\left(\theta_{t} L_{\epsilon}^{=}+\varrho \theta_{t} L_{\epsilon}^{\neq}\right)} \mathbb{1}_{\theta_{t} C_{\epsilon}=b} \mid C_{t}=c, \theta_{t} X\right]-\widetilde{M}_{t}(b) .
\end{aligned}
$$

The probability of two color changes in time $\epsilon$ is small and the above is equal to

$$
\begin{aligned}
& \widetilde{M}_{t}(b)\left(\mathbb{E}\left[e^{\gamma\left(\theta_{t} L_{\epsilon}^{=}+\varrho \theta_{t} L_{\epsilon}^{\neq}\right)} \mathbb{1}_{\theta_{t} C_{\epsilon}=b} \mid C_{t}=b, \theta_{t} X\right]-1\right) \\
& +\sum_{c: d(c, b)=1} \widetilde{M}_{t}(c) \mathbb{E}\left[e^{\gamma\left(\theta_{t} L_{\epsilon}^{=}+\varrho \theta_{t} L_{\epsilon}^{\neq}\right)} \mathbb{1}_{\theta_{t} C_{\epsilon}=b} \mid C_{t}=c, \theta_{t} X\right]+O\left(\kappa^{2}\right) \\
& =\widetilde{M}_{t}(b)\left(e^{\gamma\left(\kappa \mathbb{1}_{b_{k}=b_{\ell}}+\varrho \kappa \mathbb{1}_{\left.b_{k} \neq b_{\ell}\right)}\right.} e^{\left.-\gamma \kappa \mathbb{1}_{b_{k}=b_{\ell}}-1\right)}\right. \\
& \quad+\mathbb{1}_{b_{k} \neq b_{\ell}} \sum_{i \in\{k, \ell\}} \widetilde{M}_{t}\left(\widehat{b}^{i}\right) e^{O(\kappa)}\left(1-e^{-\frac{\gamma}{2} \kappa}\right)+O\left(\kappa^{2}\right) .
\end{aligned}
$$


By writing $e^{x}=1+x+O\left(x^{2}\right)$, we get

$$
\begin{aligned}
& \frac{\gamma \varrho}{2} \mathbb{1}_{b_{k} \neq b_{\ell}} \widetilde{M}_{t}(b) \kappa+\frac{\gamma}{2} \mathbb{1}_{b_{k} \neq b_{\ell}} \sum_{i \in\{k, \ell\}} \widetilde{M}_{t}\left(\widehat{b}^{i}\right) \kappa+O\left(\kappa^{2}\right) \\
& =\frac{\gamma \varrho}{2} \sum_{i, j=1}^{n} \mathbb{1}_{b_{i} \neq b_{j}} \widetilde{M}_{t}(b)\left(L_{t+\epsilon}^{i, j}-L_{t}^{i, j}\right) \\
& \quad+\frac{\gamma}{2} \sum_{i, j=1}^{n} \mathbb{1}_{b_{i} \neq b_{j}} \widetilde{M}_{t}\left(\widehat{b}^{i}\right)\left(L_{t+\epsilon}^{i, j}-L_{t}^{i, j}\right) \\
& +O\left(\left(L_{t+\epsilon}^{k, \ell}-L_{t}^{k, \ell}\right)^{2}\right) .
\end{aligned}
$$

Sending $\epsilon$ to 0 completes the proof.

4. The moment duality for $\gamma=\infty$. In this section, we will derive the moment duality for the case $\gamma=\infty$ and thus prove Theorems 2.3 and 2.5. We start by looking at the discrete space case in Section 4.1. In particular, we introduce an auxiliary process $K$, whose asymptotics we analyze in Section 4.2. We then complete the proof of Theorem 2.3 (discrete space) in Section 4.3. The continuous space case is slightly different, and we prove Theorem 2.3 in this setting in Section 4.4. Finally, we combine Theorem 2.3 with a dominated convergence argument to show the moment duality for $\gamma=\infty$, that is, Theorem 2.5, in Section 4.5.

Since the case $n=1$ is trivial, we will assume throughout that $n \geq 2$. Also, we can treat the case $\varrho=-1$ simultaneously with the case $\varrho \in(-1,0)$.

4.1. Analysis of $M^{[\gamma]}$ for the discrete space model. In this section, we assume that $X=\left(X^{(1)}, \ldots, X^{(n)}\right)$ is a simple, symmetric random walk on $\left(\mathbb{Z}^{d}\right)^{n}$ with total jump rate 1 . Remember that given the paths of $X$, the process $M_{t}^{[\gamma]}=M_{t}^{[\gamma]}\left(X, M_{0}\right)$ is defined as the solution of

$$
\begin{aligned}
\frac{d}{d t} M_{t}^{[\gamma]}(m)= & \frac{\gamma \varrho}{2} \sum_{i, j=1}^{n} \mathbb{1}_{m_{i} \neq m_{j}} M_{t}^{[\gamma]}(m) \frac{d L_{t}^{i, j}}{d t} \\
& +\frac{\gamma}{2} \sum_{i, j=1}^{n} \mathbb{1}_{m_{i} \neq m_{j}} M_{t}^{[\gamma]}\left(\widehat{m}^{i}\right) \frac{d L_{t}^{i, j}}{d t},
\end{aligned}
$$

for each coloring $m \in\{1,2\}^{n}$, with initial condition $M_{0} \in \mathcal{M}\left(\{1,2\}^{n}\right)$, where we recall that $L_{t}^{i, j}$ denote the pair local times and $\widehat{m}^{i}$ is the coloring $m$ flipped at $i$.

We notice that the right-hand side of (12) is piecewise linear. To make this precise, we introduce the following notation: For a partition $\pi$ of the set $[n]:=$ $\{1, \ldots, n\}$ we write $\pi=\left\{\pi_{1}, \pi_{2}, \ldots, \pi_{k}\right\}$ (in increasing order of the smallest element), where $\pi_{i} \subset[n]$ with size $\left|\pi_{i}\right|$ are the blocks of $\pi$, and we define $|\pi|:=k$. 
Also, if $M$ is a measure on $\{1,2\}^{n}$ we interpret $M=(M(m))_{m \in\{1,2\}^{n}}$ as a $2^{n}$ dimensional vector by ordering the elements of $\{1,2\}^{n}$ in increasing lexicographical order.

For a partition $\pi$ of $[n]$, let $A_{\varrho}^{\pi}$ be defined as the $2^{n}$-dimensional matrix with nonzero entries given by

$$
A_{\varrho}^{\pi}\left(m, m^{\prime}\right)= \begin{cases}\frac{\varrho}{2} \sum_{k=1}^{|\pi|} \sum_{i, j \in \pi_{k}} \mathbb{1}_{m_{i} \neq m_{j}} & \text { if } m^{\prime}=m, \\ \frac{1}{2} \sum_{j \in \pi_{k}} \mathbb{1}_{m_{i} \neq m_{j}} & \text { if } m^{\prime}=\widehat{m}^{i} \text { for some } i \in \pi_{k}, k \in[|\pi|] .\end{cases}
$$

Then we can rewrite (12) as (see also Lemma 3.2)

$$
\frac{d}{d t} M_{t}^{[\gamma]}(m)=\gamma A_{\varrho}^{\pi\left(X_{t}\right)} M_{t}^{[\gamma]}(m),
$$

where we take $\pi\left(X_{t}\right)$ as the set of clusters of the random walks $X_{t}$, that is, $\pi\left(X_{t}\right)$ is the partition of $[n]$ induced by the equivalence relation $i \sim j$ iff $X_{t}^{(i)}=X_{t}^{(j)}$.

In the discrete space case, $\pi\left(X_{t}\right)$ is piecewise constant, which motivates us to first study the evolution under $A_{\varrho}^{\pi}$ for a fixed partition $\pi$ of $[n]$. Let $K_{t}\left(K_{0}, \pi\right)=$ $e^{t A_{\varrho}^{\pi}} K_{0}$ be the unique solution of

$$
\frac{d}{d t}\left(K_{t}\left(K_{0}, \pi\right)\right)=A_{\varrho}^{\pi} K_{t}\left(K_{0}, \pi\right)
$$

with initial value $K_{0} \in \mathcal{M}\left(\{1,2\}^{n}\right)$. From (13) it is clear that $M_{t}^{[\gamma]}$ can be directly constructed from $K_{t}$, as follows:

LEMMA 4.1. Let $\tau_{0}:=0$ and $\tau_{k}:=\inf \left\{t \geq \tau_{k-1}: \pi\left(X_{t}\right) \neq \pi\left(X_{\tau_{k-1}}\right)\right\}, k \in \mathbb{N}$, be the times when the partition induced by the random walk changes. Then $M_{t}^{[\gamma]}$ is given recursively as $M_{0}^{[\gamma]}=M_{0}$ and for $k \in \mathbb{N}_{0}$,

$$
M_{t}^{[\gamma]}=K_{\gamma\left(t-\tau_{k}\right)}\left(M_{\tau_{k}}^{[\gamma]}, \pi\left(X_{\tau_{k}}\right)\right), \quad t \in\left[\tau_{k}, \tau_{k+1}\right] .
$$

4.2. The asymptotic analysis of $K_{t}$. To obtain the limit as $\gamma \rightarrow \infty$, the explicit form of $M_{t}^{[\gamma]}$ given by Lemma 4.1 suggests that we need to understand the limit as $t \rightarrow \infty$ of $K_{t}(\cdot, \pi)$ first. Surprisingly, we see that the critical curve (3) for the moments also appears here via an independent derivation.

We begin by introducing some additional notation: For $n \in \mathbb{N}$ and a coloring $m \in\{1,2\}^{n}$, we write

$$
\#_{a} m:=\sum_{j=1}^{n} \mathbb{1}_{m_{j}=a}, \quad a \in\{1,2\} .
$$


If in addition $\pi=\left\{\pi_{1}, \ldots, \pi_{k}\right\}$ is a partition of $[n]$ with length $|\pi|=k$, we let $\left.\left.m\right|_{\pi_{i}} \in\{1,2\}\right\}^{\left|\pi_{i}\right|}$ denote the restriction of $m$ to the block $\pi_{i}, i=1, \ldots, k$. Moreover, given $c \in\{1,2\}^{k}$ we define $m^{\pi, c} \in\{1,2\}^{n}$ by

$$
m_{j}^{\pi, c}:=c_{i} \quad \text { iff } \quad j \in \pi_{i}, \quad j=1, \ldots, n .
$$

PROPOSITION 4.2. Suppose $\varrho+\cos (\pi / n)<0$, and let $\pi$ be an arbitrary partition of $[n]$. Then there exists $K_{\infty}\left(K_{0}, \pi\right) \in \mathcal{M}\left(\{1,2\}^{n}\right)$ such that for the unique solution $K_{t}\left(K_{0}, \pi\right)$ of (14) we have

$$
\lim _{t \rightarrow \infty} K_{t}\left(K_{0}, \pi\right)=K_{\infty}\left(K_{0}, \pi\right)
$$

Moreover, the limit $K_{\infty}$ is given explicitly as follows: For $\varrho \in(-1,0)$, we have

$$
K_{\infty}\left(K_{0}, \pi\right)(m)=\sum_{c \in\{1,2\}|\pi|} K_{0}\left(m^{\pi, c}\right) \prod_{i=1}^{|\pi|} \frac{\sin \left(\left.\lambda_{\varrho} \#_{c_{i}} m\right|_{\pi_{i}}\right)}{\sin \left(\lambda_{\varrho}\left|\pi_{i}\right|\right)}, \quad m \in\{1,2\}^{n},
$$

where $\lambda_{\varrho}:=\arccos (|\varrho|)$. For $\varrho=-1$, the limit is given by

$$
K_{\infty}\left(K_{0}, \pi\right)(m)=\sum_{c \in\{1,2\}|\pi|} K_{0}\left(m^{\pi, c}\right) \prod_{i=1}^{|\pi|} \frac{\left.\#_{c_{i}} m\right|_{\pi_{i}}}{\left|\pi_{i}\right|}, \quad m \in\{1,2\}^{n} .
$$

It turns out that we can reduce the case of a general partition to the case of the partition $\pi=\{[n]\}$. Intuitively, the reason is that since there is no interaction between different blocks $\pi_{i}$ of the partition $\pi=\left\{\pi_{1}, \ldots, \pi_{k}\right\}$, the solution $K_{t}$ of (14) evolves independently on each block. Assume for simplicity that the partition $\pi$ is given by consecutive intervals, that is

$$
\pi=\left\{\left\{1, \ldots,\left|\pi_{1}\right|\right\}, \ldots,\left\{n-\left|\pi_{k}\right|+1, \ldots, n\right\}\right\} .
$$

By our convention on representing the elements of $\mathcal{M}\left(\{1,2\}^{n}\right)$ as $2^{n}$ dimensional vectors, we can write

$$
A_{\varrho}^{\pi}=A_{\varrho}^{\left[\left|\pi_{1}\right|\right]} \oplus A_{\varrho}^{\left[\left|\pi_{2}\right|\right]} \oplus \cdots \oplus A_{\varrho}^{\left[\left|\pi_{k}\right|\right]} .
$$

Here $\oplus$ denotes the Kronecker sum for matrices (see, e.g., [15], Chapter 4.4), and $A_{\varrho}^{[\ell]}, \ell \in \mathbb{N}$, is the $2^{\ell} \times 2^{\ell}$ matrix with nonzero entries defined by

$$
A_{\varrho}^{[\ell]}\left(m, m^{\prime}\right)= \begin{cases}\frac{1}{2} \varrho \sum_{i, j=1}^{\ell} \mathbb{1}_{m_{i} \neq m_{j}} & \text { if } m^{\prime}=m, \\ \frac{1}{2} \sum_{j=1}^{\ell} \mathbb{1}_{m_{i} \neq m_{j}} & \text { if } m^{\prime}=\widehat{m}^{i} \text { for some } i \in[\ell],\end{cases}
$$

for $m, m^{\prime} \in\{1,2\}^{\ell}$. In general, $\pi$ is not ordered as assumed above, so there is an additional permutation of coordinates involved, which however is only a change of basis and has no influence on the dynamics.

We start with the analysis in the simpler case. 
Proposition 4.3. Suppose $\varrho+\cos (\pi / n)<0$. Then there exists $K_{\infty}\left(K_{0}\right.$, $\{[n]\})$ such that

$$
\lim _{t \rightarrow \infty} K_{t}\left(K_{0},\{[n]\}\right)=K_{\infty}\left(K_{0},\{[n]\}\right)
$$

Moreover, for $\varrho \in(-1,0)$ the limit $K_{\infty}$ is given explicitly by

$$
K_{\infty}\left(K_{0},\{[n]\}\right)(m)=K_{0}\left((\underline{1})_{n}\right) \frac{\sin \left(\lambda_{\varrho} \#_{1} m\right)}{\sin \left(\lambda_{\varrho} n\right)}+K_{0}\left((\underline{2})_{n}\right) \frac{\sin \left(\lambda_{\varrho} \#_{2} m\right)}{\sin \left(\lambda_{\varrho} n\right)}
$$

where $\lambda_{\varrho}=\arccos (|\varrho|)$ and $(\underline{i})_{n}:=(i, \ldots, i) \in\{1,2\}^{n}$ for $i \in\{1,2\}$. For $\varrho=-1$, the limit is

$$
K_{\infty}\left(K_{0},\{[n]\}\right)(m)=K_{0}\left((\underline{1})_{n}\right) \frac{\#_{1} m}{n}+K_{0}\left((\underline{2})_{n}\right) \frac{\#_{2} m}{n} .
$$

In order to prove Proposition 4.3, we start with a lemma that analyzes the eigenvalues of the matrix $A_{\varrho}^{[n]}$ defined in (16).

LEMMA 4.4. The matrix $A_{\varrho}^{[n]}$ has eigenvalue 0 of multiplicity (at least) 2, and the remaining $2^{n}-2$ eigenvalues have negative (resp. non-positive) real part iff $\varrho+\cos (\pi / n)<0($ resp. $\leq 0)$.

ProOF. For ease of notation, we write $A:=A_{\varrho}^{[n]}$, also since $n \geq 2$ we note that $\varrho \leq 0$. Note that the first and last rows in $A$ are zero rows, in particular $A$ has eigenvalue 0 of multiplicity at least two. Moreover, if we define $A^{\prime}$ by removing the first row and column and the last row and column, then the eigenvalues of $A$ are 0 (twice) and those of $A^{\prime}$.

For $k \in\{1, \ldots, n-1\}$, define $\mathcal{I}^{k}:=\left\{m \in\{1,2\}^{n}: \#_{1} m=k\right\}$. We start by looking for eigenvectors of $A$ that are constant for coordinates $m \in \mathcal{I}^{k}$, in which case will write $\tilde{v}_{k}=v(m)$ for $m \in \mathcal{I}^{k}$.

Note that for $m \in \mathcal{I}^{k}$

$$
A\left(m, m^{\prime}\right)= \begin{cases}\varrho k(n-k) & \text { if } m=m^{\prime}, \\ \frac{1}{2}(n-k) & \text { if } m^{\prime}=\widehat{m}^{i} \text { and } m_{i}=1, \\ \frac{1}{2} k & \text { if } m^{\prime}=\widehat{m}^{i} \text { and } m_{i}=2 .\end{cases}
$$


Hence, by setting $\tilde{v}_{0}:=\tilde{v}_{n}:=0$ we get for $m \in \mathcal{I}^{k}, k \in\{1, \ldots, n-1\}$ that

$$
\begin{aligned}
A v(m) & =\varrho k(n-k) v(m)+\sum_{i \in[n]} A\left(m, \widehat{m}^{i}\right) v\left(\widehat{m}^{i}\right) \\
& =\varrho k(n-k) \tilde{v}_{k}+\frac{1}{2} \sum_{i \in[n]}(n-k) \mathbb{1}_{m_{i}=1} v\left(\widehat{m}^{i}\right)+\frac{1}{2} \sum_{i \in[n]} k \mathbb{1}_{m_{i}=2} v\left(\widehat{m}^{i}\right) \\
& =\varrho k(n-k) \tilde{v}_{k}+\frac{1}{2} \sum_{i \in[n]}(n-k) \tilde{v}_{k-1} \mathbb{1}_{m_{i}=1}+\frac{1}{2} \sum_{i \in[n]} k \mathbb{1}_{m_{i}=2} \tilde{v}_{k+1} \\
& =\varrho k(n-k) \tilde{v}_{k}+\frac{1}{2} k(n-k) \tilde{v}_{k-1}+\frac{1}{2}(n-k) k \tilde{v}_{k+1} \\
& =\tilde{A} \tilde{v},
\end{aligned}
$$

where we define $\tilde{A}$ as the $(n-1) \times(n-1)$ matrix with non-zero entries

$$
\tilde{A}(k, \ell):=\left\{\begin{array}{ll}
\frac{1}{2} k(n-k) & \text { if } \ell=k-1, \\
\varrho k(n-k) & \text { if } \ell=k, \\
\frac{1}{2} k(n-k) & \text { if } \ell=k+1,
\end{array} \quad k, \ell \in[n-1] .\right.
$$

Clearly, $\tilde{A}$ can be written as $\tilde{A}=D B$, where $D$ is the diagonal matrix with entries $k(n-k)$ for $k \in[n-1]$, and $B$ is a tridiagonal Toeplitz matrix with diagonal entries $\varrho$ and off-diagonal entries $1 / 2$. It is well known (and can be checked easily) that $B$ has eigenvalues $\varrho+\cos (j \pi / n), j \in[n-1]$.

It is clear that $\tilde{A}=D B$ has the same eigenvalues as $D^{1 / 2} B D^{1 / 2}$ (in fact these two matrices are similar by a diagonal change of basis), and the latter is a symmetric matrix. Moreover, if $\varrho+\cos (\pi / n)<0$ (resp. $\leq 0)$, then $B$ is negative (semi-) definite, therefore for any vector $w \neq 0$

$$
w^{T} D^{1 / 2} B D^{1 / 2} w=\left(D^{1 / 2} w\right)^{T} B\left(D^{1 / 2} w\right)<0 \quad(\text { resp. } \leq 0) .
$$

In this case, since $D^{1 / 2} B D^{1 / 2}$ is symmetric, all its eigenvalues are real and negative (resp. non-positive). Hence, under this condition the same is true for $\tilde{A}$. In particular, its largest eigenvalue $\lambda_{*}$ is negative (non-positive).

Let $\tilde{v}^{*}$ be an eigenvector corresponding to the eigenvalue $\lambda_{*}$ of $\tilde{A}$. We will use the Perron-Frobenius theorem to argue that $\tilde{v}^{*}$ has positive coordinates. Observing that only the diagonal entries of $\tilde{A}$ are non-positive, we can choose some suitable constant $c>0$ such that $\tilde{A}^{*}:=\tilde{A}+c I_{n-1}$ is a non-negative matrix that is irreducible and aperiodic. In particular, the Perron-Frobenius theorem applies to $\tilde{A}^{*}$. Moreover, w.l.o.g. the constant $c>0$ can be taken so large that the spectrum of $\tilde{A}^{*}$ is contained in $[0, \infty)$. Then since $\lambda_{*}$ is the largest eigenvalue of $\tilde{A}$, we have that $c+\lambda_{*}$ is the largest eigenvalue and spectral radius of $\tilde{A}^{*}$. Since $\tilde{v}^{*}$ is a corresponding eigenvector, by Perron-Frobenius it must have positive coordinates. 
Coming back to the matrix $A^{\prime}$ obtained from $A$ by removing the first row and column and the last row and column, we can define a $\left(2^{n}-2\right)$-dimensional vector $v^{\prime}$ by setting $v^{\prime}(m):=\tilde{v}_{k}^{*}$ if $m \in \mathcal{I}^{k}, k \in[n-1]$. Then by definition of $A^{\prime}$ and by (17), we see that $v^{\prime}$ is an eigenvector for $A^{\prime}$ with eigenvalue $\lambda_{*}$. Thus, it is also an eigenvector for $A^{*}:=A^{\prime}+c I_{2^{n}-2}$ with eigenvalue $c+\lambda_{*}$, where $c>0$ is chosen as above. Since Perron-Frobenius applies to $A^{*}$ and the vector $v^{\prime}$ is strictly positive, $c+\lambda_{*}$ must also be the spectral radius of $A^{*}$. Now let $\lambda \in \mathbb{C}$ be any eigenvalue of $A^{\prime}$. Then $c+\lambda$ is an eigenvalue of $A^{*}$, and we get

$$
\operatorname{Re}(\lambda)=\operatorname{Re}(c+\lambda)-c \leq|c+\lambda|-c \leq\left(c+\lambda_{*}\right)-c=\lambda_{*} .
$$

Finally, since the eigenvalues of $A$ are 0 (with multiplicity 2) together with those of $A^{\prime}$, we have proved the statement of the lemma.

We will now prove Proposition 4.3 by identifying the eigenvectors of $A_{\varrho}^{[n]}$ corresponding to eigenvalue 0 . For $k \in\{0, \ldots, n\}$, we consider again $\mathcal{I}^{k}:=\{m \in$ $\left.\{1,2\}^{n}: \#_{1} m=k\right\}$. Then we define two vectors $v_{j}^{(n)}=\left(v_{j}^{(n)}(m)\right)_{m \in\{1,2\}^{n}} \in \mathbb{R}^{2^{n}}$ $(j=1,2)$ as follows: If $\varrho=-1$, we set

$$
v_{1}^{(n)}(m):=\frac{k}{n} \quad \text { and } \quad v_{2}^{(n)}(m):=\frac{n-k}{n} \quad \text { if } m \in \mathcal{I}^{k},
$$

while for $\varrho>-1, \varrho+\cos (\pi / n)<0$ we set

$$
v_{1}^{(n)}(m):=\frac{\sin \left(\lambda_{\varrho} k\right)}{\sin \left(\lambda_{\varrho} n\right)} \quad \text { and } \quad v_{2}^{(n)}(m):=\frac{\sin \left(\lambda_{\varrho}(n-k)\right)}{\sin \left(\lambda_{\varrho} n\right)} \quad \text { if } m \in \mathcal{I}^{k},
$$

where $\lambda_{\varrho}:=\arccos (|\varrho|)$. Observe that by construction, we have $v_{1}^{(n)}\left((\underline{1})_{n}\right)=$ $v_{2}^{(n)}\left((\underline{2})_{n}\right)=1$ and $v_{2}^{(n)}\left(\left(\underline{1}_{n}\right)=v_{1}^{(n)}\left((\underline{2})_{n}\right)=0\right.$. Also note that the explicit form of the limit claimed in Proposition 4.3 can be restated as

$$
\lim _{t \rightarrow \infty} e^{t A_{\varrho}^{[n]}}=\left(v_{1}^{(n)}, 0, \ldots, 0, v_{2}^{(n)}\right),
$$

where the RHS is a matrix consisting of the vectors $v_{1}^{(n)}$ resp. $v_{2}^{(n)}$ in the first resp. last column and zeros otherwise.

ProOF OF Proposition 4.3. We will prove the proposition by showing that $v_{1}^{(n)}$ and $v_{2}^{(n)}$ are eigenvectors of $A_{\varrho}^{[n]}$ corresponding to eigenvalue 0 , and also that $K_{t}:=K_{t}\left(K_{0},\{[n]\}\right)$ satisfies

$$
\lim _{t \rightarrow \infty} K_{t}=K_{0}\left((\underline{1})_{n}\right) v_{1}^{(n)}+K_{0}\left((2)_{n}\right) v_{2}^{(n)} .
$$

Let us assume that we have already shown that $v_{1}^{(n)}$ and $v_{2}^{(n)}$ are eigenvectors of $A:=A_{\varrho}^{[n]}$ corresponding to eigenvalue 0 , so that in particular $e^{t A} v_{j}^{(n)}=v_{j}^{(n)}$, 
$j=1,2$. Then, we will show the stronger statement that there exists $C>0$ and $\lambda_{0}>0$ such that

$$
\left\|e^{t A}-\left(v_{1}^{(n)}, 0, \ldots, 0, v_{2}^{(n)}\right)\right\| \leq C e^{-\lambda_{0} t}
$$

for all $t \geq 0$ and $\|\cdot\|$, for example, denoting the matrix (operator) norm induced by the Euclidean norm on $\mathbb{R}^{2^{n}}$, which readily implies (20).

We let $A^{\prime}$ be defined as in the proof of Lemma 4.4. Then, since by Lemma 4.4 all its eigenvalues have negative real part, there exists $C^{\prime}>0$ and $\lambda_{0}>0$ such that $\left\|e^{t A^{\prime}}\right\| \leq C^{\prime} e^{-\lambda_{0} t}$ for all $t \geq 0$. Let $w_{3}, \ldots, w_{2^{n}}$ be an orthogonal basis for $\mathbb{R}^{2^{n}-2}$. We define $v_{k}^{(n)}$ for $k \geq 3$ by extending the vector $w_{k}$ at either side by zero entries to obtain a $2^{n}$-dimensional vector [so in particular $v_{k}^{(n)}\left((\underline{1})_{n}\right)=v_{k}^{(n)}\left((2)_{n}\right)=0$, $k \geq 3]$. Together with the vectors $v_{1}^{(n)}, v_{2}^{(n)}$ this forms a basis of $\mathbb{R}^{2^{n}}$. Hence, we write any vector in $\mathbb{R}^{2^{n}}$ as $K_{0}=\sum_{i} \mu_{i} v_{i}^{(n)}$ for some coefficients $\mu_{i}$. Furthermore, $A$ acts on $v_{k}^{(n)}$ as $A^{\prime}$ acts on $w_{k}, k=3, \ldots, 2^{n}$, in the corresponding bases:

$$
\left\langle A v_{k}^{(n)}, v_{j}^{(n)}\right\rangle=\left\langle A^{\prime} w_{k}, w_{j}\right\rangle, \quad j, k=3, \ldots, 2^{n} .
$$

Therefore, $\left\|e^{t A} v_{k}^{(n)}\right\| \leq C^{\prime} e^{-\lambda_{0} t}\left\|v_{k}^{(n)}\right\|$ for $k \geq 3$ and so there exists $C>0$ such that for all $t \geq 0$,

$$
\begin{aligned}
\left\|e^{t A} K_{0}-\left(\mu_{1} v_{1}^{(n)}+\mu_{2} v_{2}^{(n)}\right)\right\| & =\left\|\sum_{i} \mu_{i} e^{t A} v_{i}^{(n)}-\left(\mu_{1} v_{1}^{(n)}+\mu_{2} v_{2}^{(n)}\right)\right\| \\
& \leq C e^{-\lambda_{0} t}\left\|K_{0}\right\| .
\end{aligned}
$$

Moreover, notice that since $v_{1}^{(n)}$ resp. $v_{2}^{(n)}$ is the only basis element with a nonzero entry in the first resp. last coordinate, we must have $\mu_{1}=K_{0}\left((\underline{1})_{n}\right)$ and $\mu_{2}=$ $K_{0}\left((2)_{n}\right)$.

Finally, it remains to show that $v_{1}^{(n)}$ and $v_{2}^{(n)}$ are eigenvectors with eigenvalue 0 . This can either be verified by direct calculation or alternatively it can be derived as follows: In order to obtain $v_{1}^{(n)}$, we look for an eigenvector $v$ that is constant for coordinates $m \in \mathcal{I}^{k}$ and such that $v\left((\underline{1})_{n}\right)=1$ and $v\left((\underline{2})_{n}\right)=0$. As in the proof of Lemma 4.4, we will write $\tilde{v}_{k}=v(m)$ for $m \in \mathcal{I}^{k}$, and $A v=0$ reduces to the recurrence equation

$$
0=\varrho k(n-k) \tilde{v}_{k}+\frac{1}{2} k(n-k) \tilde{v}_{k-1}+\frac{1}{2}(n-k) k \tilde{v}_{k+1} ;
$$

see (17). Assuming that $\varrho \in(-1,0)$, this equation has solution $\tilde{v}_{k}=c_{1} t_{1}^{k}+c_{2} t_{2}^{k}$, where $t_{1}$ and $t_{2}$ are the distinct roots of the equation

$$
t^{2}+2 \varrho t+1=0 .
$$


Thus since $\varrho<0, t_{1,2}=-\varrho \pm i \sqrt{1-\varrho^{2}}=e^{ \pm \lambda \varrho i}$, where $\lambda_{\varrho}:=\arctan \left(\frac{\sqrt{1-\varrho^{2}}}{|\varrho|}\right)=$ $\arccos (|\varrho|)$. Simplifying further, we note that since $\tilde{v}_{0}=0$, we have $c_{1}=-c_{2}$ and thus $\tilde{v}_{k}=c_{1} 2 i \sin \left(\lambda_{\varrho} k\right)$. Therefore, since $\tilde{v}_{n}=1$, we get $c_{1}=\frac{1}{2 i \sin \left(\lambda_{\varrho} n\right)}$, so that

$$
\tilde{v}_{k}=\frac{\sin \left(\lambda_{\varrho} k\right)}{\sin \left(\lambda_{\varrho} n\right)} \text {. }
$$

Moreover, we note that this expression is positive for $k \in\{1, \ldots, n-1\}$, since $\lambda_{\varrho} n<\pi$ iff $\varrho+\cos \pi / n<0$.

In the case that $\varrho=-1$, the characteristic equation (22) has the unique solution $t=1$ and so the solution to the recurrence equation is given by $\tilde{v}_{k}=c_{1}+c_{2} k$ for suitable constants $c_{1}, c_{2}$, which by using the boundary conditions reduces to $\tilde{v}_{k}=k / n$.

In either case, defining $v(m):=\tilde{v}_{k}$ for $m \in \mathcal{I}^{k}$ gives the first eigenvector $v_{1}^{(n)}$ with eigenvalue 0 . The derivation of the second eigenvector $v_{2}^{(n)}$ is analogous.

Using the decomposition (15), we can now use Proposition 4.3 to analyze the dynamics of $K_{t}$ in the case of a general partition.

ProOF OF Proposition 4.2. Throughout we write $K_{t}=K_{t}\left(K_{0}, \pi\right)$. We first assume that $\pi=\left\{\left\{1, \ldots, n_{1}\right\},\left\{n_{1}+1, \ldots, n_{2}\right\}, \ldots,\left\{n_{1}+\cdots+n_{k-1}+1, \ldots, n_{k}\right\}\right\}$ with $\sum_{i=1}^{k} n_{i}=n$. Then, by the decomposition (15) and the fact that $e^{t(\dot{A} \oplus B)}=$ $e^{t A} \otimes e^{t \bar{B}}$, we have that

$$
K_{t}=e^{t A_{\varrho}^{\pi}} K_{0}=\bigotimes_{i=1}^{k} e^{t A_{\varrho}^{\left[n_{i}\right]}} K_{0} .
$$

Therefore, since $\varrho+\cos (\pi / n)<0$ implies $\varrho+\cos \left(\pi / n_{i}\right)<0$ for all $i \in[k]$, we obtain from Proposition 4.3 [see also (21)] that there exists $C>0$ and $\lambda_{0}>0$ such that for all $t \geq 0$

$$
\left\|e^{t A_{\varrho}^{\pi}}-\bigotimes_{i=1}^{k}\left(v_{1}^{\left(n_{i}\right)}, 0, \ldots, 0, v_{2}^{\left(n_{i}\right)}\right)\right\| \leq C e^{-\lambda_{0} t} .
$$

Also, we can identify the limit as

$$
\begin{aligned}
& \bigotimes_{i=1}^{k}\left(v_{1}^{\left(n_{i}\right)}, 0, \ldots, 0, v_{2}^{\left(n_{i}\right)}\right) K_{0} \\
& \quad=\sum_{c_{1}, \ldots, c_{k} \in\{1,2\}} K_{0}\left(\left(\underline{c}_{1}\right)_{n_{1}}, \ldots,\left(\underline{c}_{k}\right)_{n_{k}}\right) v_{c_{1}}^{\left(n_{1}\right)} \otimes \cdots \otimes v_{c_{k}}^{\left(n_{k}\right)},
\end{aligned}
$$

where we write

$$
\left(\underline{c}_{1}\right)_{n_{1}}, \ldots,\left(\underline{c}_{k}\right)_{n_{k}}:=(\underbrace{c_{1}, \ldots, c_{1}}_{n_{1} \text { times }}, \ldots, \underbrace{c_{k}, \ldots, c_{k}}_{n_{k} \text { times }}) \in\{1,2\}^{n} .
$$


In view of the definition of the eigenvectors $v_{1}^{\left(n_{i}\right)}$ and $v_{2}^{\left(n_{i}\right)}$ [see (18) and (19)], this concludes the proof of Proposition 4.2 for the case of a partition consisting of consecutive intervals.

The case of general $\pi=\left\{\pi_{1}, \ldots, \pi_{k}\right\}$ (written in increasing order of smallest element) follows by permuting the entries in $m \in\{1,2\}^{n}$.

4.3. Proof of Theorem 2.3 in the discrete case. In discrete space, Theorem 2.3 is a direct consequence of the following proposition.

Proposition 4.5. Suppose $\varrho+\cos (\pi / n)<0$, and consider $M^{[\gamma]}:=$ $M^{[\gamma]}\left(X, M_{0}\right)$ for $M_{0} \in \mathcal{M}\left(\{1,2\}^{n}\right)$. Then, a.s. with respect to the random walk $X=\left(X^{(1)}, \ldots, X^{(n)}\right)$, for any $t \geq 0, M_{t}^{[\gamma]}$ converges to a limit $M_{t}^{[\infty]}$ as $\gamma \rightarrow \infty$. The limit $M_{t}^{[\infty]}$ is given recursively as follows: Let $\tau_{k}$ be defined as in Lemma 4.1, that is, $\tau_{0}:=0$ and $\tau_{k}, k \geq 1$ are the times when the partition $\pi\left(X_{t}\right)$ induced by the random walk changes. Then $M_{0}^{[\infty]}=M_{0}$ and

$$
M_{t}^{[\infty]}=K_{\infty}\left(M_{\tau_{k}}^{[\infty]}, \pi\left(X_{\tau_{k}}\right)\right) \quad \text { for all } t \in\left(\tau_{k}, \tau_{k+1}\right], k \in \mathbb{N}_{0},
$$

where $K_{\infty}\left(K_{0}, \pi\right)$ is given in Proposition 4.2.

PROOF. We know that almost surely, the sequence $\left(\tau_{k}\right)_{k \geq 0}$ is strictly increasing. Also, by Lemma 4.1 we know that the process $M_{t}^{[\gamma]}$ is given by

$$
M_{t}^{[\gamma]}=K_{\gamma\left(t-\tau_{k}\right)}\left(M_{\tau_{k}}^{[\gamma]}, \pi\left(X_{\tau_{k}}\right)\right), \quad t \in\left[\tau_{k}, \tau_{k+1}\right] .
$$

Then by Proposition 4.2 [and in particular (23)], we can argue inductively by using that for each $k \in \mathbb{N}_{0}$, there exists $C>0$ such that

$$
\begin{aligned}
\| M_{t}^{[\gamma]} & -K_{\infty}\left(M_{\tau_{k}}^{[\infty]}, \pi\left(X_{\tau_{k}}\right)\right) \| \\
\leq & \left\|K_{\gamma\left(t-\tau_{k}\right)}\left(M_{\tau_{k}}^{[\gamma]}, \pi\left(X_{\tau_{k}}\right)\right)-K_{\gamma\left(t-\tau_{k}\right)}\left(M_{\tau_{k}}^{[\infty]}, \pi\left(X_{\tau_{k}}\right)\right)\right\| \\
& +\left\|K_{\gamma\left(t-\tau_{k}\right)}\left(M_{\tau_{k}}^{[\infty]}, \pi\left(X_{\tau_{k}}\right)\right)-K_{\infty}\left(M_{\tau_{k}}^{[\infty]}, \pi\left(X_{\tau_{k}}\right)\right)\right\| \\
\leq & C\left\|M_{\tau_{k}}^{[\gamma]}-M_{\tau_{k}}^{[\infty]}\right\|+\left\|K_{\gamma\left(t-\tau_{k}\right)}\left(M_{\tau_{k}}^{[\infty]}, \pi\left(X_{\tau_{k}}\right)\right)-K_{\infty}\left(M_{\tau_{k}}^{[\infty]}, \pi\left(X_{\tau_{k}}\right)\right)\right\|
\end{aligned}
$$

and the LHS $\rightarrow 0$ as $\gamma \rightarrow \infty$ for all $t \in\left(\tau_{k}, \tau_{k+1}\right]$.

4.4. Proof of Theorem 2.3 in the continuous case. The continuous space case is slightly different from the discrete space case. However, the assumption that the $n$-dimensional Brownian motion $X=\left(X_{t}^{(1)}, \ldots, X_{t}^{(n)}\right)_{t \geq 0}$ starts in $\mathbf{x}=\left(x_{1}, \ldots, x_{n}\right)$ with $x_{i} \neq x_{j}$ for $i \neq j$ simplifies the situation somewhat, since we only have intersections of at most two Brownian motions simultaneously. In order to formalize this, we need to consider the times of new pair collisions between the Brownian motions. More precisely, as in Section 4.1 we write $\pi\left(X_{t}\right)$ for 
the partition of $[n]$ induced by the equivalence relation $i \sim j$ iff $X_{t}^{(i)}=X_{t}^{(j)}$. Then we define $\tau_{0}:=0$ and $\tau_{k+1}$ as the first time $t \geq \tau_{k}$ so that the partition $\pi\left(X_{t}\right)$ is not a refinement of the partition $\pi\left(X_{\tau_{k}}\right)$. The partition $\pi\left(X_{\tau_{k}}\right)$ contains for $k \geq 1$ a single pair, and otherwise singletons. Denote the pair by $\kappa_{k}=\left\{\kappa_{k, 1}, \kappa_{k, 2}\right\}$. The only refinements of $\pi\left(X_{\tau_{k}}\right)$ are the partition itself and the partition consisting only of singletons. So $\tau_{k+1}$ is the first time when there is a new collision with a collision pair $\kappa_{k+1}$ not identical to $\kappa_{k}$. By the path properties of Brownian motion, almost surely we have $\tau_{0}<\tau_{1}<\cdots$.

With this notation, we can directly deduce Theorem 2.3 in the continuous space setting from the following proposition.

Proposition 4.6. Let $X=\left(X^{(1)}, \ldots, X^{(n)}\right)$ be a Brownian motion started in $\mathbf{x} \in \mathbb{R}^{n}$ such that $x_{i} \neq x_{j}$ for $i \neq j$, and consider $M^{[\gamma]}:=M^{[\gamma]}\left(X, M_{0}\right)$ for $M_{0} \in \mathcal{M}\left(\{1,2\}^{n}\right)$. Suppose $\varrho+\cos (\pi / n)<0$. Then, a.s. with respect to $X$, for any $t \geq 0, M_{t}^{[\gamma]}$ converges to a limit $M_{t}^{[\infty]}$. The limit is given recursively as $M_{t}^{[\infty]}=M_{0}$ for $t \in\left[0, \tau_{1}\right]$ and

$$
M_{t}^{[\infty]}=K_{\infty}\left(M_{\tau_{k}}^{[\infty]}, \pi\left(X_{\tau_{k}}\right)\right) \quad \text { for all } t \in\left(\tau_{k}, \tau_{k+1}\right], k \in \mathbb{N},
$$

where $K_{\infty}\left(K_{0}, \pi\right)$ is given in Proposition 4.2.

PROOF. We recall the equation (12) for $M^{[\gamma]}$,

$$
\begin{aligned}
d M_{t}^{[\gamma]}(m)= & \frac{\gamma \varrho}{2} \sum_{i, j=1}^{n} \mathbb{1}_{m_{i} \neq m_{j}} M_{t}^{[\gamma]}(m) d L_{t}^{i, j} \\
& +\frac{\gamma}{2} \sum_{i, j=1}^{n} \mathbb{1}_{m_{i} \neq m_{j}} M_{t}^{[\gamma]}\left(\widehat{m}^{i}\right) d L_{t}^{i, j} .
\end{aligned}
$$

We notice that $M_{t}^{[\gamma]}$ is constant equal to $M_{0}$ on $\left[0, \tau_{1}\right]$. For $k \geq 1$, if we write

$$
L_{t}^{\left(\kappa_{k}\right)}:=L_{t}^{\kappa_{k, 1}, \kappa_{k, 2}}
$$

then on the interval $\left[\tau_{k}, \tau_{k+1}\right]$ equation (24) simplifies to

$$
\begin{aligned}
d M_{t}^{[\gamma]}(m)= & \gamma \varrho \mathbb{1}_{m_{\kappa_{k, 1}} \neq m_{\kappa_{k, 2}}} M_{t}^{[\gamma]}(m) d L_{t}^{\left(\kappa_{k}\right)} \\
& +\frac{\gamma}{2} \mathbb{1}_{m_{\kappa_{k, 1}} \neq m_{\kappa_{k, 2}}}\left(M_{t}^{[\gamma]}\left(\widehat{m}^{\kappa_{k, 1}}\right)+M_{t}^{[\gamma]}\left(\widehat{m}^{\kappa_{k, 2}}\right)\right) d L_{t}^{\left(\kappa_{k}\right)} .
\end{aligned}
$$

Hence, the analogue of Lemma 4.1 in the continuous space setting is that $M^{[\gamma]}$ solves $M_{t}^{[\gamma]}=M_{0}$ for $t \in\left[0, \tau_{1}\right]$ and for $k \in \mathbb{N}$,

$$
M_{t}^{[\gamma]}=K_{\gamma\left(L_{t}^{\left(\kappa_{k}\right)}-L_{\tau_{k}}^{\left(\kappa_{k}\right)}\right)}\left(M_{\tau_{k}}^{[\gamma]}, \pi\left(X_{\tau_{k}}\right)\right), \quad t \in\left[\tau_{k}, \tau_{k+1}\right]
$$

where $K_{t}$ is defined by (14) and $\pi\left(X_{\tau_{k}}\right)$ is the partition of $[n]$ consisting only of singletons, except for the pair $\kappa_{k}=\left\{\kappa_{k, 1}, \kappa_{k, 2}\right\}$. 
Finally, we argue as in the proof of Proposition 4.5, noting that by the path properties of Brownian motion, almost surely, for any $t>\tau_{k}$, we have $L_{t}^{\left(\kappa_{k}\right)}-$ $L_{\tau_{k}}^{\left(\kappa_{k}\right)}>0$.

4.5. Proof of Theorem 2.5. In order to show Theorem 2.5, we use a dominated convergence argument, so that the main technical point is to show that the processes $M_{t}^{[\gamma]}$ are uniformly bounded in $\gamma$.

Proposition 4.7. Assume $\varrho+\cos (\pi / n)<0$. Fix $M_{0} \in \mathcal{M}\left(\{1,2\}^{n}\right)$. In both the discrete and the continuous space setting, the process $M^{[\gamma]}=\left(M_{t}^{[\gamma]}(X\right.$, $\left.\left.M_{0}\right)\right)_{t \geq 0}$ remains bounded uniformly in $\gamma, t$ and $\omega$.

We precede the proof by three lemmas showing that the process $\left(K_{t}\right)_{t \geq 0}$ defined in (14) remains bounded. For this, the crucial observation is the following (which we prove in the next two lemmas): The vectors $v_{1}^{(n)}$ and $v_{2}^{(n)}$ from (18)(19), which we recall are eigenvectors with eigenvalue 0 of the matrix $A_{\varrho}^{[n]}$ from (16), corresponding to the trivial partition $\pi=\{[n]\}$, are in fact also eigenvectors with eigenvalue 0 of $A_{\varrho}^{\pi}$, for any partition $\pi$ of $[n]$.

Lemma 4.8. Assume $\varrho+\cos (\pi / n)<0$. Then, for any partition $\pi=$ $\left\{\pi_{1}, \ldots, \pi_{k}\right\}$ of $[n]$ consisting purely of singletons except for one block $\pi_{i}$, $i \in\{1, \ldots, k\}$, we have

$$
K_{\infty}\left(v_{j}^{(n)}, \pi\right)=v_{j}^{(n)}, \quad j=1,2 .
$$

PROof. Assume first $\varrho>-1$. W.l.o.g. we can assume that $i=1$ and $\pi_{1}=[\ell]$, $1 \leq \ell<n$. Let $m \in\{1,2\}^{n}$. Decomposing $m=\left(m^{\prime}, m^{\prime \prime}\right)$ with $m^{\prime} \in\{1,2\}^{\ell}$ and $m^{\prime \prime} \in\{1,2\}^{n-\ell}$, we get by Proposition 4.2 that for $j=1,2$

$$
\begin{aligned}
& K_{\infty}\left(v_{j}^{(n)}, \pi\right)(m) \\
& \quad=\sum_{c \in\{1,2\}|\pi|} v_{j}^{(n)}\left(m^{\pi, c}\right) \prod_{i=1}^{|\pi|} \frac{\sin \left(\left.\lambda_{\varrho} \#_{c_{i}} m\right|_{\pi_{i}}\right)}{\sin \left(\lambda_{\varrho}\left|\pi_{i}\right|\right)} \\
& \quad=v_{j}^{(n)}\left((\underline{1})_{\ell}, m^{\prime \prime}\right) \frac{\sin \left(\lambda_{\varrho} \#_{1} m^{\prime}\right)}{\sin \left(\lambda_{\varrho} \ell\right)}+v_{j}^{(n)}\left((\underline{2})_{\ell}, m^{\prime \prime}\right) \frac{\sin \left(\lambda_{\varrho} \#_{2} m^{\prime}\right)}{\sin \left(\lambda_{\varrho} \ell\right)},
\end{aligned}
$$

where we used that all blocks $\pi_{i}, i \geq 2$, of the partition $\pi$ consist of singletons. For $j=1$, plugging in the definition of $v_{1}^{(n)}$ from (19) we obtain that the above is equal to

$$
\frac{\sin \left(\lambda_{\varrho} \#_{1} m^{\prime}\right)}{\sin \left(\lambda_{\varrho} \ell\right)} \frac{\sin \left(\lambda_{\varrho}\left(\ell+\#_{1} m^{\prime \prime}\right)\right)}{\sin \left(\lambda_{\varrho} n\right)}+\frac{\sin \left(\lambda_{\varrho}\left(\ell-\#_{1} m^{\prime}\right)\right)}{\sin \left(\lambda_{\varrho} \ell\right)} \frac{\sin \left(\lambda_{\varrho} \#_{1} m^{\prime \prime}\right)}{\sin \left(\lambda_{\varrho} n\right)} .
$$


Now the claim is that the above equals $\frac{\sin \left(\lambda_{\varrho}\left(\#_{1} m^{\prime}+\#_{1} m^{\prime \prime}\right)\right)}{\sin \left(\lambda_{\varrho} n\right)}$. After multiplying everything with $\sin \left(\lambda_{\varrho} \ell\right) \sin \left(\lambda_{\varrho} n\right)$, this follows directly from the addition and subtraction theorems for the sine function applied to the three sines with a sum or difference as argument. The proof for the second eigenvector $v_{2}^{(n)}$ is analogous.

For $\varrho=-1$ the strategy is the same, but because of the different structure of the vectors $v_{j}^{(n)}$, the function $x \mapsto \sin \left(\lambda_{\varrho} x\right)$ needs to be replaced by the identity.

LEMMA 4.9. Assume $\varrho+\cos (\pi / n)<0$. Then for any partition $\pi=$ $\left\{\pi_{1}, \ldots, \pi_{k}\right\}$ of $[n]$ we have $K_{\infty}\left(v_{j}^{(n)}, \pi\right)=v_{j}^{(n)}, j=1,2$.

PROOF. The linear map $K_{\infty}(\cdot, \pi)$ is the Kronecker product of the $K_{\infty}\left(\cdot, \pi_{i}\right)$, $i=1, \ldots, k$, up to relabeling of the coordinates. This implies that

$$
K_{\infty}(\cdot, \pi)=K_{\infty}\left(\cdot, \tilde{\pi}_{1}\right) \circ K_{\infty}\left(\cdot, \tilde{\pi}_{2}\right) \circ \cdots \circ K_{\infty}\left(\cdot, \tilde{\pi}_{k}\right),
$$

where $\tilde{\pi}_{i}$ is the partition of $[n]$ which consists of $\pi_{i}$ and otherwise singletons. The claim then follows from Lemma 4.8 .

LEMMA 4.10. Assume $\varrho+\cos (\pi / n)<0$. Given $a_{1}, a_{2} \geq 0$, define $V_{n}^{a_{1}, a_{2}} \in$ $\mathcal{M}\left(\{1,2\}^{n}\right)$ via $V_{n}^{a_{1}, a_{2}}:=a_{1} v_{1}^{(n)}+a_{2} v_{2}^{(n)}$. Then for any partition $\pi=\left\{\pi_{1}, \ldots, \pi_{k}\right\}$ of $[n]$ and any $K_{0} \in \mathcal{M}\left(\{1,2\}^{n}\right)$ such that $0 \leq K_{0} \leq V_{n}^{a_{1}, a_{2}}$ coordinate wise, we have

$$
K_{t}\left(K_{0}, \pi\right) \leq V_{n}^{a_{1}, a_{2}}, \quad t>0
$$

ProOF. Write $V_{n}:=V_{n}^{a_{1}, a_{2}}$ for short. Let $W$ be such that $0 \leq W \leq V_{n}$ coordinatewise. Note that $A_{\varrho}^{\pi}$ has only nonnegative off-diagonal entries, therefore $e^{t A_{\varrho}^{\pi}}$ has only non-negative entries. Moreover, $V_{n}$ is an eigenvector of $A_{\varrho}^{\pi}$ with respect to eigenvalue 0 , so that

$$
0 \leq e^{t A_{\varrho}^{\pi}}\left(V_{n}-W\right)=V_{n}-e^{t A_{\varrho}^{\pi}} W .
$$

In particular, whenever $K_{0} \leq V_{n}$, the solution of (14) satisfies $K_{t}\left(K_{0}, \pi\right) \leq$ $V_{n}$.

Proof of Proposition 4.7. Given $M_{0} \in \mathcal{M}\left(\{1,2\}^{n}\right)$, we can always find $a_{1}, a_{2} \geq 0$ such that $M_{0} \leq a_{1} v_{1}^{(n)}+a_{2} v_{2}^{(n)}=V_{n}^{a_{1}, a_{2}}$ holds coordinatewise. Then we can combine Lemma 4.10 with the representation of $M_{t}^{[\gamma]}$ in terms of $K_{t}$; see Lemma 4.1 (discrete space) and (25) (continuous space), to get the bound $M_{t}^{[\gamma]} \leq V_{n}^{a_{1}, a_{2}}$ inductively on each time interval $\left[\tau_{k}, \tau_{k+1}\right], k \in \mathbb{N}$.

Equipped with these uniform bounds, we can now prove the moment duality for the infinite rate models by combining Theorem 2.3 with a dominated convergence argument. 
ProOf OF THEOREM 2.5. We give the proof for the continuous-space case $\mathcal{S}=\mathbb{R}$. Suppose first that $\phi=\bigotimes_{i=1}^{n} \phi_{i}$ with $\phi_{i} \in \mathcal{C}_{c}^{+}(\mathbb{R}), i=1, \ldots, n$. Rewriting the moment duality for finite $\gamma>0$ in weak form, by Theorem 2.1 and Fubini we have for all $t>0$ that

$$
\begin{aligned}
\mathbb{E}_{\mathbf{u}_{0}}\left[\int_{\mathbb{R}^{n}} \bigotimes_{i=1}^{n} \phi_{i}(\mathbf{x}) H\left(\mathbf{u}_{t}^{[\gamma]} ; \mathbf{x}, c\right) d \mathbf{x}\right] \\
\quad=\int_{\mathbb{R}^{n}} \bigotimes_{i=1}^{n} \phi_{i}(\mathbf{x}) \mathbb{E}_{\mathbf{x}}\left[\sum_{b \in\{1,2\}^{n}} M_{t}^{[\gamma]}\left(X, \delta_{c}\right)(b) \mathbf{u}_{0}^{(b)}\left(X_{t}\right)\right] d \mathbf{x} .
\end{aligned}
$$

By Theorem 2.3, we know that for Lebesgue-a.e. $\mathbf{x} \in \mathbb{R}^{n}$, almost surely under $\mathbb{P}_{\mathbf{x}}$, for any $t \geq 0, M_{t}^{[\gamma]}\left(X, \delta_{c}\right)$ converges to $M_{t}^{[\infty]}\left(X, \delta_{c}\right)$ as $\gamma \rightarrow \infty$. Since $\mathbf{u}_{0}$ is bounded by assumption and $M_{t}^{[\gamma]}$ is bounded uniformly in $\gamma$ (and $\omega$ ) by Proposition 4.7, we can deduce by dominated convergence that the right-hand side converges to

$$
\int_{\mathbb{R}^{n}} \bigotimes_{i=1}^{n} \phi_{i}(\mathbf{x}) \mathbb{E}_{\mathbf{x}}\left[\sum_{b \in\{1,2\}^{n}} M_{t}^{[\infty]}\left(X, \delta_{c}\right)(b) \mathbf{u}_{0}^{(b)}\left(X_{t}\right)\right] d \mathbf{x}, \quad t>0 .
$$

For the left-hand side, we know that $\left(\mathbf{u}_{t}^{[\gamma]}\right)_{t \geq 0} \rightarrow\left(\mathbf{u}_{t}\right)_{t \geq 0}$ as $\gamma \uparrow \infty$ in $D_{[0, \infty)}\left(\mathcal{M}_{\text {tem }}(\mathbb{R})^{2}\right)$ w.r.t. the Meyer-Zheng topology. By [20], Theorem 1.1, Corollary 1.4, we may and do assume that there is a sequence $\gamma_{k} \uparrow \infty$ and a subset $I \subseteq \mathbb{R}^{+}$of full Lebesgue measure such that for all $t \in I$ we have $\mathbf{u}_{t}^{\left[\gamma_{k}\right]} \rightarrow \mathbf{u}_{t}$ as $k \rightarrow \infty$ in $\mathcal{M}_{\text {tem }}(\mathbb{R})^{2}$, almost surely. Recalling the definition of the topology on $\mathcal{M}_{\text {tem }}(\mathbb{R})$ (see, e.g., the beginning of Section 2, or [4], Appendix A.1), we then have also

$$
\int_{\mathbb{R}^{n}} \bigotimes_{i=1}^{n} \phi_{i}(\mathbf{x}) H\left(\mathbf{u}_{t}^{\left[\gamma_{k}\right]} ; \mathbf{x}, c\right) d \mathbf{x} \stackrel{k \rightarrow \infty}{\longrightarrow} \prod_{i=1}^{n}\left\langle u_{t}^{\left(c_{i}\right)}, \phi_{i}\right\rangle=\int_{\mathbb{R}^{n}} \bigotimes_{i=1}^{n} \phi_{i}(\mathbf{x}) \mathbf{u}_{t}^{(c)}(d \mathbf{x}),
$$

$t \in I$, almost surely. Further, note that since $\varrho+\cos (\pi / n)<0$ we are strictly below the critical curve defined in (3). In particular, we can find $\varepsilon>0$ such that the $(n+\varepsilon)$ th moments of $\operatorname{SBM}(\varrho, \gamma)_{\mathbf{u}_{0}}$ remain bounded uniformly in $\gamma$, cf. [13], Corollary 3.8. By uniform integrability, we conclude that the convergence (26) holds also in expectation, proving the moment duality (6) for all $t \in I$ and test functions of the considered form. In order to extend it to all $t>0$, choose a sequence $t_{n} \in I$ with $t_{n} \downarrow t$ and use the right-continuity of the paths of $\left(\mathbf{u}_{t}\right)_{t \geq 0}$ together with the fact that $(n+\varepsilon)$ th moments of $\operatorname{SBM}(\varrho, \infty) \mathbf{u}_{0}$ are bounded uniformly on compact time intervals, cf. [13], Proposition 2.8. Now the fact that (6) holds for all test functions $\phi=\bigotimes_{i=1}^{n} \phi_{i}$ with $\phi_{i} \in \mathcal{C}_{c}^{+}(\mathbb{R})$ shows in particular that the measure $\mathbb{E}_{\mathbf{u}_{0}}\left[\mathbf{u}_{t}^{(c)}(d x)\right]$ on $\mathbb{R}^{n}$ is absolutely continuous with a bounded Lebesgue density. Thus, the extension to arbitrary $\phi \in L^{1}\left(\mathbb{R}^{n}\right)$ follows by a standard approximation argument. 
The proof for the discrete-space case $\mathcal{S}=\mathbb{Z}^{d}$ is analogous. In fact it is even simpler, since we can avoid using test functions and instead argue pointwise.

\subsection{Proof of Corollary 2.6.}

Proof. We first observe that $A_{\varrho}^{\{11\},\{2\}\}}=0$ and hence

$$
K_{t}\left(K_{0},\{\{1\},\{2\}\}\right) \equiv K_{0} .
$$

Moreover, since

$$
A_{\varrho}^{\{1,2\}\}}=A_{\varrho}^{[2]}=\left(\begin{array}{cccc}
0 & 0 & 0 & 0 \\
\frac{1}{2} & \varrho & 0 & \frac{1}{2} \\
\frac{1}{2} & 0 & \varrho & \frac{1}{2} \\
0 & 0 & 0 & 0
\end{array}\right)
$$

we obtain that $\varrho$ is an eigenvalue of $A_{\varrho}^{\{11,2\}\}}$ and $\delta_{(1,2)}$ a corresponding eigenvector. Hence,

$$
K_{t}\left(\delta_{(1,2)},\{\{1,2\}\}\right)=e^{\varrho t} \delta_{(1,2)} .
$$

In view of Propositions 4.5 and 4.6, this implies

$$
M_{t}^{[\infty]}\left(X, \delta_{(1,2)}\right)=\mathbb{1}_{t \leq \tau_{1}} \delta_{(1,2)} \quad \text { if } X \text { starts from }(x, y) \in \mathcal{S}^{2}, x \neq y
$$

and in the discrete case also

$$
M_{t}^{[\infty]}\left(X, \delta_{(1,2)}\right) \equiv 0 \quad \text { if } X \text { starts from }(x, x) \in\left(\mathbb{Z}^{d}\right)^{2} .
$$

From this it follows easily that for second mixed moments, the duality (6) can be rewritten in the form (7), proving part (i) of the corollary.

For part (ii), we obtain from (27)-(28) and Lemma 4.1 that

$$
M_{t}^{[\gamma]}\left(X, \delta_{(1,2)}\right)=K_{\gamma L_{t}^{1,2}}\left(\delta_{(1,2)},\{\{1,2\}\}\right)=e^{\varrho \gamma L_{t}^{1,2}} \delta_{(1,2)}, \quad t \geq 0 .
$$

Therefore, by the reformulation of the finite rate moment duality (Theorem 2.1) we have

$$
\begin{aligned}
\mathbb{E}_{\mathbf{u}_{0}}\left[u_{t}^{(1)}(x) u_{t}^{(2)}(x)\right] & \\
= & \mathbb{E}_{(x, x)}\left[\sum_{m \in\{1,2\}^{2}} M_{t}^{[\gamma]}\left(X, \delta_{(1,2)}\right)(m) \mathbf{u}_{0}^{(m)}\left(X_{t}\right)\right] \\
& =\mathbb{E}_{(x, x)}\left[e^{\varrho \gamma L_{t}^{1,2}} u_{0}^{(1)}\left(X_{t}^{(1)}\right) u_{0}^{(2)}\left(X_{t}^{(2)}\right)\right]
\end{aligned}
$$

for all $x \in \mathbb{Z}^{d}$. By the transience of $Y_{t}:=X_{t}^{(1)}-X_{t}^{(2)}$, there is a geometric number of returns of $\left(Y_{t}\right)_{t \geq 0}$ to 0 with parameter $1-p_{d}$, where $p_{d}<1$ is the return 
probability of a random walk in $\mathbb{Z}^{d}$. Whenever $Y_{t}$ arrives in 0 it waits an exponentially distributed time with parameter 2 [since $X_{t}^{(1)}$ or $X_{t}^{(2)}$ could jump]. Therefore, $L_{\infty}^{1,2}:=\lim _{t \rightarrow \infty} L_{t}^{1,2}$ is a geometric number of independent exponentials, which is an exponential random variable $Z$ with parameter $2\left(1-p_{d}\right)$. Together, we obtain

$$
\begin{aligned}
\mathbb{E}_{\mathbf{u}_{0}}\left[u_{t}^{(1)}(x) u_{t}^{(2)}(x)\right] \\
\quad \leq\left\|u_{0}^{(1)}\right\|_{\infty}\left\|u_{0}^{(2)}\right\|_{\infty} \mathbb{E}\left[e^{\varrho \gamma Z}\right] \\
\quad= \begin{cases}\left\|u_{0}^{(1)}\right\|_{\infty}\left\|u_{0}^{(2)}\right\|_{\infty}\left(1-\frac{\varrho \gamma}{2\left(1-p_{d}\right)}\right)^{-1}, & \frac{\varrho \gamma}{2\left(1-p_{d}\right)}<1, \\
\infty, & \frac{\varrho \gamma}{2\left(1-p_{d}\right)} \geq 1,\end{cases}
\end{aligned}
$$

with the first inequality being asymptotically, as $t \rightarrow \infty$, an equality if $\mathbf{u}_{0}=$ $(\mathbb{1}, \mathbb{1})$.

5. The analysis of $\operatorname{SBM}(-1, \infty)$. In this section, we prove all the results for the case $\varrho=-1$. In particular, in Section 5.1 we show Theorem 2.8, establishing the existence of an infinite rate limit for $\varrho=-1$ and general initial conditions which was so far open in both discrete and continuous space. The limit is uniquely characterized by the moment duality (6) and the Markov property. In the subsequent subsections, we move on to a more explicit description of the limit in the continuous-space case. In Section 5.2, we collect some preliminary lemmas which will be needed in the sequel. We then show in Section 5.3 that the number of interfaces is non-increasing. Then, we prove Theorem 2.10, the explicit characterization of the limit in the single interface type, in Section 5.4. The extension to at most countably many interfaces without accumulation point, Theorem 2.12 , is in Section 5.5, while the proof for general initial conditions, Theorem 2.14, is in Section 5.6.

5.1. Proof of Theorem 2.8. The proof of Theorem 2.8 will be given by a series of lemmas and propositions. We first show in Proposition 5.1 tightness (w.r.t. the Meyer-Zheng topology) of the family of finite rate models $\operatorname{SBM}(-1, \gamma)$, considered as measure-valued processes, and that each limit point satisfies the separation of types-property and the moment duality (6), which uniquely determines the onedimensional distributions. From this, uniqueness of limit points and thus convergence will follow once we have established the Markov property of subsequential limit points. Thus in Proposition 5.3, we show that the one-dimensional distributions form a Markov semigroup and prove the respective Feller properties as stated in Theorem 2.8. In the continuous space case $\mathcal{S}=\mathbb{R}$, this allows us to strengthen the convergence result to hold in the stronger Skorokhod topology and to obtain continuous paths for the limiting process. 
Let $\mathcal{S} \in\left\{\mathbb{R}, \mathbb{Z}^{d}\right\}$ and $K>0$. Recall the notation $\mathcal{M}_{K}(\mathcal{S})$ and $\mathcal{M}_{K}^{\text {sep }}(\mathcal{S})$ introduced before Theorem 2.8. Throughout this section, we consider initial densities $\mathbf{u}=\left(u^{(1)}, u^{(2)}\right) \in \mathcal{M}_{K}(\mathcal{S})$ for the finite rate model. For $\gamma \in(0, \infty)$, let $\left(\mathbf{u}_{t}^{[\gamma]}\right)_{t \geq 0} \in \mathcal{C}_{[0, \infty)}\left(\mathcal{M}_{K}(\mathcal{S})\right)$ denote the solution to $\operatorname{SBM}(-1, \gamma)_{\mathbf{u}}$, considered as measure-valued process, and $\mathbb{P}_{\mathbf{u}}$ resp. $\mathbb{E}_{\mathbf{u}}$ the corresponding probability law resp. expectation. Recall that since $\varrho=-1$, the sum of the solutions evolves as $w_{t}:=S_{t} w_{0}$, the solution to the deterministic heat equation with initial condition $w_{0}:=u^{(1)}+u^{(2)}$, where $\left(S_{t}\right)_{t \geq 0}$ denotes the heat semigroup.

Proposition 5.1. For all $\mathbf{u} \in \mathcal{M}_{K}(\mathcal{S})$, the family of processes $\left\{\left(\mathbf{u}_{t}^{[\gamma]}\right)_{t \geq 0}\right.$ : $\gamma>0\}$ is tight under $\mathbb{P}_{\mathbf{u}}$ w.r.t. the Meyer-Zheng topology on the space $D_{[0, \infty)}\left(\mathcal{M}_{K}(\mathcal{S})\right)$. Moreover, each subsequential limit point $\left(\mathbf{u}_{t}\right)_{t \geq 0} \in$ $D_{[0, \infty)}\left(\mathcal{M}_{K}(\mathcal{S})\right)$ satisfies the moment duality (6) and the separation of typesproperty for positive times, that is, for each $t>0$ we have, almost surely, $\mathbf{u}_{t} \in \mathcal{M}_{K}^{\mathrm{sep}}(\mathcal{S})$.

Proof. By [4], Proposition 3.8, for the continuous case $\mathcal{S}=\mathbb{R}$ resp. [13], Proposition 3.1, for the discrete case $\mathcal{S}=\mathbb{Z}^{d}$, we know that the family of processes $\left\{\left(\mathbf{u}_{t}^{[\gamma]}\right)_{t \geq 0}: \gamma>0\right\}$ is tight under $\mathbb{P}_{\mathbf{u}}$ w.r.t. the Meyer-Zheng topology on the space $D_{[0, \infty)}\left(\mathcal{M}_{\text {tem }}(\mathcal{S})^{2}\right)$. In fact, these results were proved for all $\varrho<0$, including the case $\varrho=-1$.

Let $\left(\mathbf{u}_{t}\right)_{t \geq 0} \in D_{[0, \infty)}\left(\mathcal{M}_{\text {tem }}(\mathcal{S})^{2}\right)$ be any subsequential limit point. Then by essentially the same proof as that of Theorem 2.5, $\left(\mathbf{u}_{t}\right)_{t>0}$ satisfies the moment duality (6) for each $n \in \mathbb{N}, t>0$. Moreover, the sum $u_{t}^{(1)}+u_{t}^{(2)}$ is deterministic and satisfies $u_{t}^{(1)}+u_{t}^{(2)}=w_{t}=S_{t} w_{0}$ with $w_{0}=u^{(1)}+u^{(2)}$, since the same is true for all finite $\gamma \in(0, \infty)$. This argument shows in particular that almost surely under $\mathbb{P}_{\mathbf{u}}$, the limit measures $u_{t}^{(1)}$ and $u_{t}^{(2)}$ are absolutely continuous for all $t>0$ (in case $\mathcal{S}=\mathbb{R}$ ) with densities bounded uniformly by $\left\|w_{0}\right\|_{\infty} \leq K$, showing in particular that $\left(\mathbf{u}_{t}\right)_{t \geq 0}$ takes values in the subspace $\mathcal{M}_{K}(\mathcal{S}) \subseteq \mathcal{M}_{\text {tem }}(\mathcal{S})^{2}$. Moreover, as in [4], Lemma 4.4, Corollary 4.5, the mutual singularity of the densities $u_{t}^{(1)}$ and $u_{t}^{(2)}$ for fixed $t>0$ (separation of types) can be deduced from the identity (7) for second mixed moments which is a special case of the moment duality (6).

We now turn to the Markov property of limit points and the Feller property of the associated semigroup. Again let $\left(\mathbf{u}_{t}\right)_{t \geq 0} \in D_{[0, \infty)}\left(\mathcal{M}_{K}(\mathcal{S})\right)$ be any subsequential limit point (w.r.t. the Meyer-Zheng topology) of the family $\left\{\left(\mathbf{u}_{t}^{[\gamma]}\right)_{t \geq 0}: \gamma>0\right\}$ under $\mathbb{P}_{\mathbf{u}}$. We define a family of probability laws on $\mathcal{M}_{K}(\mathcal{S})$ as follows: For $t>0$ and $\mathbf{u} \in \mathcal{M}_{K}(\mathcal{S})$, let $P_{t}(\mathbf{u}, \cdot)$ be the law of $\mathbf{u}_{t}$ under $\mathbb{P}_{\mathbf{u}}$, that is,

$$
P_{t}(\mathbf{u}, f):=P_{t} f(\mathbf{u}):=\mathbb{E}_{\mathbf{u}}\left[f\left(\mathbf{u}_{t}\right)\right]
$$

for bounded measurable functions $f: \mathcal{M}_{K}(\mathcal{S}) \rightarrow \mathcal{S}$. We extend the definition (30) to $t=0$ by setting $P_{0}(\mathbf{u}, \cdot):=\delta_{\mathbf{u}}(\cdot)$. Since the one-dimensional distributions of 
$\left(\mathbf{u}_{t}\right)_{t \geq 0}$ are uniquely determined by the moment duality, the definition of $P_{t}$ does not depend on the choice of limit point. Also note that since $\left(\mathbf{u}_{t}\right)_{t \geq 0}$ satisfies the separation of types-property for positive times, the law $P_{t}(\mathbf{u}, \cdot)$ is concentrated on $\mathcal{M}_{K}^{\mathrm{sep}}(\mathcal{S})$ for all $t>0$.

REMARK 5.2. We stress that the suggestive notation (30) notwithstanding, we do not know a priori that $P_{t}(\cdot, \cdot)$ is a transition kernel for any fixed $t>0$, let alone that the family $\left(P_{t}(\cdot, \cdot)\right)_{t \geq 0}$ defines a Markov semigroup. Also note that since convergence in the Meyer-Zheng topology does not imply convergence of finite dimensional distributions, a priori there is no reason why the (càdlàg!) limit point $\left(\mathbf{u}_{t}\right)_{t \geq 0}$ should start from the same initial condition as the approximating finite rate processes, that is, it is not clear whether

$$
\lim _{t \downarrow 0} \mathbf{u}_{t}=\mathbf{u} \quad \text { under } \mathbb{P}_{\mathbf{u}}
$$

In fact, we know by Proposition 5.1 that $\mathbf{u}_{t} \in \mathcal{M}_{K}^{\mathrm{sep}}(\mathcal{S})$ for all $t>0$. Since $\mathcal{M}_{K}^{\text {sep }}\left(\mathbb{Z}^{d}\right)$ is closed in $\mathcal{M}_{K}\left(\mathbb{Z}^{d}\right)$, in the discrete space case (31) can only hold if the initial measures $\mathbf{u}$ for the finite rate processes are already mutually singular. This means that for initial conditions $\mathbf{u} \in \mathcal{M}_{K}\left(\mathbb{Z}^{d}\right) \backslash \mathcal{M}_{K}^{\mathrm{sep}}\left(\mathbb{Z}^{d}\right)$, the two limits $\gamma \uparrow \infty$ and $t \downarrow 0$ do not interchange. Note that this is also reflected in the behavior of the dual process $\left(M_{t}^{[\infty]}\right)_{t \geq 0}$ from Theorem 2.3: By Proposition 4.5, we have $\lim _{t \downarrow 0} M_{t}^{[\infty]}\left(X, M_{0}\right)=K_{\infty}\left(M_{0}, \pi\left(X_{0}\right)\right)$, which need not coincide with $M_{0}$ if $\pi\left(X_{0}\right)$ is not the partition into singletons.

However, we will see below that (31) indeed holds for all $\mathbf{u} \in \mathcal{M}_{K}^{\text {sep }}\left(\mathbb{Z}^{d}\right)$, as well as for all $\mathbf{u} \in \mathcal{M}_{K}(\mathbb{R})$ in the continuous space case. ${ }^{5}$

We summarize our results on Markov and Feller properties in the following proposition, where we denote by $\left(P_{t}^{[\gamma]}\right)_{t \geq 0}$ the semigroup of the finite rate symbiotic branching model $\operatorname{SBM}(-1, \gamma)$.

Proposition 5.3. Let $\mathcal{S} \in\left\{\mathbb{R}, \mathbb{Z}^{d}\right\}$ and $K>0$.

(a) For each $\gamma>0,\left(P_{t}^{[\gamma]}\right)_{t \geq 0}$ is a Feller semigroup on $\mathcal{M}_{K}(\mathcal{S})$.

(b) The family of probability laws $\left(P_{t}\right)_{t \geq 0}$ from (30) defines a Markov semigroup on $\mathcal{M}_{K}(\mathcal{S})$ such that

for all $\mathbf{u} \in \mathcal{M}_{K}(\mathcal{S})$ and $t>0: P_{t}(\mathbf{u}, \cdot)$ is concentrated on $\mathcal{M}_{K}^{\mathrm{sep}}(\mathcal{S})$.

Moreover, the semigroup $\left(P_{t}\right)_{t \geq 0}$ satisfies

$$
P_{t}\left(\mathcal{C}\left(\mathcal{M}_{K}(\mathcal{S})\right)\right) \subseteq \mathcal{C}\left(\mathcal{M}_{K}(\mathcal{S})\right), \quad t>0,
$$

\footnotetext{
${ }^{5}$ This is not a contradiction since in the continuous case, $\mathcal{M}_{K}^{\text {sep }}(\mathbb{R})$ is not closed but a dense subset of $\mathcal{M}_{K}(\mathbb{R})$.
} 
and we have the strong convergence

$$
\sup _{\mathbf{u} \in \mathcal{M}_{K}(\mathcal{S})}\left|P_{t} f(\mathbf{u})-P_{t}^{[\gamma]} f(\mathbf{u})\right| \rightarrow 0 \quad \text { as } \gamma \rightarrow \infty,
$$

for each $f \in \mathcal{C}\left(\mathcal{M}_{K}(\mathcal{S})\right)$ and $t>0$.

(c) In the continuous space case $\mathcal{S}=\mathbb{R}$, we have

$$
\sup _{\mathbf{u} \in \mathcal{M}_{K}(\mathbb{R})}\left|P_{t} f(\mathbf{u})-f(\mathbf{u})\right| \rightarrow 0 \quad \text { as } t \downarrow 0
$$

for all $f \in \mathcal{C}\left(\mathcal{M}_{K}(\mathbb{R})\right)$. In particular, $\left(P_{t}\right)_{t \geq 0}$ is a Feller semigroup on $\mathcal{C}\left(\mathcal{M}_{K}(\mathbb{R})\right)$.

(d) In the discrete space case $\mathcal{S}=\mathbb{Z}^{d}$, we have

$$
\sup _{\mathbf{u} \in \mathcal{M}_{K}^{\text {sep }}\left(\mathbb{Z}^{d}\right)}\left|P_{t} f(\mathbf{u})-f(\mathbf{u})\right| \rightarrow 0 \quad \text { as } t \downarrow 0
$$

for all $f \in \mathcal{C}\left(\mathcal{M}_{K}^{\mathrm{sep}}\left(\mathbb{Z}^{d}\right)\right)$. In particular, $\left(P_{t}\right)_{t \geq 0}$ is a Feller semigroup when restricted to the subspace $\mathcal{M}_{K}^{\mathrm{sep}}\left(\mathbb{Z}^{d}\right)$. On the space $\mathcal{M}_{K}\left(\mathbb{Z}^{d}\right)$, the semigroup $\left(P_{t}\right)_{t \geq 0}$ is not right-continuous at $t=0$, hence not a Feller semigroup.

The key to the proof of Proposition 5.3 is the next lemma, for which we need the following notation: Given $n \in \mathbb{N}, m \in\{1,2\}^{n}$ and $\phi \in L^{1}\left(\mathcal{S}^{n}\right)$ we define a function $f_{\phi, m}: \mathcal{M}_{K}(\mathcal{S}) \rightarrow \mathbb{R}$ by

$$
f_{\phi, m}(\mathbf{u}):=\int_{\mathbb{R}^{n}} \mathbf{u}^{(m)}(\mathbf{x}) \phi(\mathbf{x}) d \mathbf{x}, \quad \mathbf{u} \in \mathcal{M}_{K}(\mathcal{S}),
$$

where $\mathbf{u}^{(m)}(\mathbf{x}):=\prod_{i=1}^{n} u^{\left(m_{i}\right)}\left(x_{i}\right)$. Note that the function $f_{\phi, m}$ is bounded on each $\mathcal{M}_{K}(\mathcal{S}), K>0$. Moreover, if $\phi$ has the form $\phi(\mathbf{x})=\prod_{i=1}^{n} \phi_{i}\left(x_{i}\right)$ with $\phi_{i} \in \mathcal{C}_{c}(\mathcal{S})$, $i=1, \ldots, n$, then by the definition of the topology in $\mathcal{M}_{\text {tem }}(\mathcal{S})$ it is clear that $f_{\phi, m}$ is continuous, in both cases $\mathcal{S} \in\left\{\mathbb{R}, \mathbb{Z}^{d}\right\}$.

LEMMA 5.4. Let $\mathcal{S} \in\left\{\mathbb{R}, \mathbb{Z}^{d}\right\}$ and $K>0$. For $n \in \mathbb{N}, m \in\{1,2\}^{n}, \phi_{i} \in \mathcal{C}_{c}(\mathcal{S})$ for $i=1, \ldots, n$ and $\phi(\mathbf{x}):=\prod_{i=1}^{n} \phi_{i}\left(x_{i}\right)$, consider the function $f_{\phi, m}$ defined in (33).

(a) For all $t>0$ and $\gamma \in(0, \infty)$, the functions $P_{t} f_{\phi, m}$ and $P_{t}^{[\gamma]} f_{\phi, m}$ are continuous on $\mathcal{M}_{K}(\mathcal{S})$, and we have

$$
\sup _{\mathbf{u} \in \mathcal{M}_{K}(\mathcal{S})}\left|P_{t} f_{\phi, m}(\mathbf{u})-P_{t}^{[\gamma]} f_{\phi, m}(\mathbf{u})\right| \rightarrow 0 \quad \text { as } \gamma \rightarrow \infty .
$$

(b) Let $\mathcal{S}=\mathbb{R}$. Then for all $\mathbf{u} \in \mathcal{M}_{K}(\mathbb{R})$ we have $P_{t} f_{\phi, m}(\mathbf{u}) \rightarrow f_{\phi, m}(\mathbf{u})$ as $t \downarrow 0$.

(c) Let $\mathcal{S}=\mathbb{Z}^{d}$. Then for all $\mathbf{u} \in \mathcal{M}_{K}\left(\mathbb{Z}^{d}\right)$ we have

$$
P_{t} f_{\phi, m}(\mathbf{u}) \rightarrow \int_{\mathcal{M}_{K}^{\mathrm{sep}}\left(\mathbb{Z}^{d}\right)} f_{\phi, m}(\tilde{\mathbf{u}}) J(\mathbf{u})(d \tilde{\mathbf{u}}) \quad \text { as } t \downarrow 0,
$$

where $J: \mathcal{M}_{K}\left(\mathbb{Z}^{d}\right) \rightarrow \mathcal{P}\left(\mathcal{M}_{K}^{\mathrm{sep}}\left(\mathbb{Z}^{d}\right)\right)$ is the function defined in (8). In particular, for all $\mathbf{u} \in \mathcal{M}_{K}^{\mathrm{sep}}\left(\mathbb{Z}^{d}\right)$ we have $P_{t} f_{\phi, m}(\mathbf{u}) \rightarrow f_{\phi, m}(\mathbf{u})$ as $t \downarrow 0$. 
PROOF. (a) Fix $t>0$. By the moment duality for finite resp. infinite branching rate, we have

$$
\begin{aligned}
P_{t}^{[\gamma]} f_{\phi, m}(\mathbf{u}) & =\sum_{m^{\prime} \in\{1,2\}^{n}} \int_{\mathbb{R}^{n}} d \mathbf{x} \phi(\mathbf{x}) \mathbb{E}_{\mathbf{x}}\left[\mathbf{u}^{\left(m^{\prime}\right)}\left(X_{t}\right) M_{t}^{[\gamma]}\left(X, \delta_{m}\right)\left(m^{\prime}\right)\right], \\
P_{t} f_{\phi, m}(\mathbf{u}) & =\sum_{m^{\prime} \in\{1,2\}^{n}} \int_{\mathbb{R}^{n}} d \mathbf{x} \phi(\mathbf{x}) \mathbb{E}_{\mathbf{x}}\left[\mathbf{u}^{\left(m^{\prime}\right)}\left(X_{t}\right) M_{t}^{[\infty]}\left(X, \delta_{m}\right)\left(m^{\prime}\right)\right] .
\end{aligned}
$$

Consequently, we obtain

$$
\begin{aligned}
& \left|P_{t}^{[\gamma]} f_{\phi, m}(\mathbf{u})-P_{t} f_{\phi, m}(\mathbf{u})\right| \\
& \quad \leq K^{n} \sum_{m^{\prime} \in\{1,2\}^{n}} \int_{\mathcal{S}^{n}} d \mathbf{x}|\phi(\mathbf{x})| \mathbb{E}_{\mathbf{x}}\left[\left|M_{t}^{[\gamma]}\left(X, \delta_{m}\right)\left(m^{\prime}\right)-M_{t}^{[\infty]}\left(X, \delta_{m}\right)\left(m^{\prime}\right)\right|\right]
\end{aligned}
$$

uniformly in $\mathbf{u} \in \mathcal{M}_{K}(\mathcal{S})$. Now we use Theorem 2.3 and Proposition 4.7 to conclude by dominated convergence that the RHS tends to 0 as $\gamma \uparrow \infty$, thus (34) is shown.

Since convergence of measures in $\mathcal{M}_{K}\left(\mathbb{Z}^{d}\right)$ implies pointwise convergence of the respective counting densities, the continuity of $P_{t} f_{\phi, m}$ and $P_{t}^{[\gamma]} f_{\phi, m}$ is immediate from (35) in the discrete space case $\mathcal{S}=\mathbb{Z}^{d}$. We now show continuity of $P_{t} f_{\phi, m}$ for the continuous space case $\mathcal{S}=\mathbb{R}$. We start by rewriting (35) as

$$
\begin{aligned}
P_{t} f_{\phi, m}(\mathbf{u}) & \\
= & \sum_{m^{\prime} \in\{1,2\}^{n}} \int_{\mathbb{R}^{n}} d \mathbf{x} \phi(\mathbf{x}) \int_{\mathbb{R}^{n}} d \mathbf{z} p_{t}(\mathbf{x}-\mathbf{z}) \mathbf{u}^{\left(m^{\prime}\right)}(\mathbf{z}) \\
& \times \mathbb{E}_{\mathbf{x}}\left[M_{t}^{[\infty]}\left(X, \delta_{m}\right)\left(m^{\prime}\right) \mid X_{t}=\mathbf{z}\right] \\
= & g_{t, \phi, m}^{(\delta)}(\mathbf{u}) \\
& +\sum_{m^{\prime} \in\{1,2\}^{n}} \int_{\mathbb{R}^{n}} d \mathbf{x} \phi(\mathbf{x}) \int_{\mathbb{R}^{n}} d \mathbf{y} \int_{\mathbb{R}^{n}} d \mathbf{z} p_{t-\delta}(\mathbf{x}-\mathbf{y}) p_{\delta}(\mathbf{y}-\mathbf{z}) \mathbf{u}^{\left(m^{\prime}\right)}(\mathbf{z}) \\
& \times\left(\mathbb{E}_{\mathbf{x}}\left[M_{t}^{[\infty]}\left(X, \delta_{m}\right)\left(m^{\prime}\right) \mid X_{t}=\mathbf{z}\right]\right. \\
& \left.-\mathbb{E}_{\mathbf{x}}\left[M_{t}^{[\infty]}\left(X, \delta_{m}\right)\left(m^{\prime}\right) \mid X_{t}=\mathbf{y}\right]\right),
\end{aligned}
$$

where we define

$$
\begin{aligned}
g_{t, \phi, m}^{(\delta)}(\mathbf{u}):= & \sum_{m^{\prime} \in\{1,2\}^{n}} \int_{\mathbb{R}^{n}} d \mathbf{x} \phi(\mathbf{x}) \int_{\mathbb{R}^{n}} d \mathbf{y} p_{t-\delta}(\mathbf{x}-\mathbf{y}) \\
& \times \mathbb{E}_{\mathbf{x}}\left[M_{t}^{[\infty]}\left(X, \delta_{m}\right)\left(m^{\prime}\right) \mid X_{t}=\mathbf{y}\right] \int_{\mathbb{R}^{n}} d \mathbf{z} p_{\delta}(\mathbf{y}-\mathbf{z}) \mathbf{u}^{\left(m^{\prime}\right)}(\mathbf{z}) .
\end{aligned}
$$


We will show that $g_{t, \phi, m}^{(\delta)}(\mathbf{u})$ is continuous as a function of $\mathbf{u} \in \mathcal{M}_{K}(\mathbb{R})$ for each fixed $\delta>0$, while the second term on the RHS of (36) tends to 0 as $\delta \downarrow 0$ uniformly in $\mathbf{u} \in \mathcal{M}_{K}(\mathbb{R})$.

First, it is clear by the definition of the topology of $\mathcal{M}_{\text {tem }}(\mathbb{R})$ that $\int_{\mathbb{R}^{n}} p_{\delta}(\mathbf{y}-$ $\mathbf{z}) \tilde{\mathbf{u}}^{\left(m^{\prime}\right)}(\mathbf{z}) d \mathbf{z} \rightarrow \int_{\mathbb{R}^{n}} p_{\delta}(\mathbf{y}-\mathbf{z}) \mathbf{u}^{\left(m^{\prime}\right)}(\mathbf{z}) d \mathbf{z}$ as $\tilde{\mathbf{u}} \rightarrow \mathbf{u}$ in $\mathcal{M}_{K}(\mathbb{R})$, for each fixed $\mathbf{y} \in \mathbb{R}^{n}$. Since the process $M_{t}^{[\infty]}$ is uniformly bounded by Proposition 4.7 and $\mathbf{u}^{\left(m^{\prime}\right)}(\mathbf{z}) \leq K^{n}$ uniformly in $\mathbf{u} \in \mathcal{M}_{K}(\mathbb{R})$ and $\mathbf{z} \in \mathbb{R}^{n}$, by dominated convergence we get that $g_{t, \phi, m}^{(\delta)}(\tilde{\mathbf{u}}) \rightarrow g_{t, \phi, m}^{(\delta)}(\mathbf{u})$ as $\tilde{\mathbf{u}} \rightarrow \mathbf{u}$ in $\mathcal{M}_{K}(\mathbb{R})$. Moreover, for each $\mathbf{u} \in \mathcal{M}_{K}(\mathbb{R})$ we have by (36) that

$$
\begin{aligned}
& \left|P_{t} f_{\phi, m}(\mathbf{u})-g_{t, \phi, m}^{(\delta)}(\mathbf{u})\right| \\
& \leq K^{n} \sum_{m^{\prime} \in\{1,2\}^{n}} \int_{\mathbb{R}^{n}} d \mathbf{x} \phi(\mathbf{x}) \int_{\mathbb{R}^{n}} d \mathbf{y} p_{t-\delta}(\mathbf{x}-\mathbf{y}) \int_{\mathbb{R}^{n}} d \mathbf{z} p_{\delta}(\mathbf{y}-\mathbf{z}) \\
& \quad \times \mid \mathbb{E}_{\mathbf{x}}\left[M_{t}^{[\infty]}\left(X, \delta_{m}\right)\left(m^{\prime}\right) \mid X_{t}=\mathbf{z}\right] \\
& \quad-\mathbb{E}_{\mathbf{x}}\left[M_{t}^{[\infty]}\left(X, \delta_{m}\right)\left(m^{\prime}\right) \mid X_{t}=\mathbf{y}\right] \mid .
\end{aligned}
$$

Since $M_{t}^{[\infty]}$ depends only on the number and order of collisions between the Brownian motions $X$, we have that $\mathbb{E}_{\mathbf{x}}\left[M_{t}^{[\infty]}\left(X, \delta_{m}\right)\left(m^{\prime}\right) \mid X_{t}=\cdot\right]$ is continuous outside of points where $y_{i}=y_{j}$ for some $i \neq j$. In particular, for almost every $\mathbf{x}, \mathbf{y} \in \mathbb{R}^{n}$ the function

$$
\mathbf{z} \mapsto\left(\mathbb{E}_{\mathbf{x}}\left[M_{t}^{[\infty]}\left(X, \delta_{m}\right)\left(m^{\prime}\right) \mid X_{t}=\mathbf{z}\right]-\mathbb{E}_{\mathbf{x}}\left[M_{t}^{[\infty]}\left(X, \delta_{m}\right)\left(m^{\prime}\right) \mid X_{t}=\mathbf{y}\right]\right)
$$

is continuous at $\mathbf{y}$, where it takes the value 0 . Thus, the integral $\int_{\mathbb{R}^{n}} d \mathbf{z} \cdots$ in (37) tends to 0 for almost every $\mathbf{x}, \mathbf{y} \in \mathbb{R}^{n}$. Again using that the process $M_{t}^{[\infty]}$ is uniformly bounded, by dominated convergence the whole integral on the RHS of (37) vanishes as $\delta \downarrow 0$. In particular, the LHS vanishes uniformly in $\mathbf{u} \in \mathcal{M}_{K}$.

Consequently, given $\varepsilon>0$ we first choose $\delta>0$ such that $\mid P_{t} f_{\phi, m}(\mathbf{u})-$ $g_{t, \phi, m}^{(\delta)}(\mathbf{u}) \mid<\varepsilon / 3$ for all $\mathbf{u} \in \mathcal{M}_{K}(\mathbb{R})$ and then $\eta=\eta(\delta)>0$ such that $\mid g_{t, \phi, m}^{(\delta)}(\mathbf{u})-$ $g_{t, \phi, m}^{(\delta)}(\tilde{\mathbf{u}}) \mid<\varepsilon / 3$ for $d_{\mathcal{M}_{\text {tem }}}(\mathbf{u}, \tilde{\mathbf{u}})<\eta$ to obtain

$$
\begin{aligned}
& \left|P_{t} f_{\phi, m}(\mathbf{u})-P_{t} f_{\phi, m}(\tilde{\mathbf{u}})\right| \\
& \leq\left|P_{t} f_{\phi, m}(\mathbf{u})-g_{t, \phi, m}^{(\delta)}(\mathbf{u})\right|+\left|g_{t, \phi, m}^{(\delta)}(\mathbf{u})-g_{t, \phi, m}^{(\delta)}(\tilde{\mathbf{u}})\right| \\
& \quad+\left|P_{t} f_{\phi, m}(\tilde{\mathbf{u}})-g_{t, \phi, m}^{(\delta)}(\tilde{\mathbf{u}})\right|
\end{aligned}
$$

which is $<\varepsilon$ whenever $d_{\mathcal{M}_{\text {tem }}}(\mathbf{u}, \tilde{\mathbf{u}})<\eta$. Thus, $P_{t} f_{\phi, m}$ is continuous on $\mathcal{M}_{K}(\mathbb{R})$. The proof for the continuity of $P_{t}^{[\gamma]} f_{\phi, m}$ is analogous, replacing $M_{t}^{[\infty]}$ by $M_{t}^{[\gamma]}$. 
(b) Let $\mathcal{S}=\mathbb{R}$. For each $\mathbf{u} \in \mathcal{M}_{K}(\mathbb{R})$, we have by the moment duality

$$
\begin{aligned}
P_{t} f_{\phi, m}(\mathbf{u})= & \int_{\mathbb{R}^{n}} \phi(\mathbf{x}) \mathbb{E}_{\mathbf{x}}\left[\sum_{m^{\prime} \in\{1,2\}^{n}} \mathbf{u}^{\left(m^{\prime}\right)}\left(X_{t}\right) M_{t}^{[\infty]}\left(X, \delta_{m}\right)\left(m^{\prime}\right)-\mathbf{u}^{(m)}\left(X_{t}\right)\right] d \mathbf{x} \\
& +\int_{\mathbb{R}^{n}} \phi(\mathbf{x}) \mathbb{E}_{\mathbf{x}}\left[\mathbf{u}^{(m)}\left(X_{t}\right)\right] d \mathbf{x} .
\end{aligned}
$$

By Proposition 4.6, we know that for Lebesgue-a.e. $\mathbf{x} \in \mathbb{R}^{n}$, almost surely under $\mathbb{P}_{\mathbf{x}}$, we have $M_{t}^{[\infty]}\left(X, \delta_{m}\right)=\delta_{m}$ for all $t>0$ small enough, whence by dominated convergence we conclude that the first term on the RHS of the last display converges to 0 as $t \downarrow 0$. Clearly, the second term converges to $f_{\phi, m}(\mathbf{u})$ as $t \downarrow 0$.

(c) For $\mathcal{S}=\mathbb{Z}^{d}$, we can argue pointwise, that is, it suffices to consider test functions of the form $\phi_{i}(\cdot)=\mathbb{1}_{x_{i}}(\cdot)$ for $x_{i} \in \mathbb{Z}^{d}$ fixed, so that $f_{\phi, m}(\mathbf{u})=\mathbf{u}^{(m)}(\mathbf{x})$. However, the additional difficulty in this case is that we can no longer assume that the $x_{i}$ are all distinct.

Fix $\mathbf{u} \in \mathcal{M}_{K}\left(\mathbb{Z}^{d}\right), m \in\{1,2\}^{n}$ and $\mathbf{x}=\left(x_{1}, \ldots, x_{n}\right) \in\left(\mathbb{Z}^{d}\right)^{n}$. Let $\pi:=\pi(\mathbf{x})$ be the partition of $[n]$ induced by the equivalence relation $i \sim j$ iff $x_{i}=x_{j}$, with length $k:=|\pi|$ and blocks $\pi_{j}, j=1, \ldots, k$. We define $\mathbf{y} \in\left(\mathbb{Z}^{d}\right)^{k}$ by

$$
y_{j}:=x_{i} \quad \text { for } i \in \pi_{j}, j=1, \ldots, k .
$$

First, we consider the case that there is a block of $\pi$ containing at least two elements such that $m$ is not constant on this block. In this case, we necessarily have $\tilde{\mathbf{u}}^{(m)}(\mathbf{x})=0$ for all $\tilde{\mathbf{u}} \in \mathcal{M}_{K}^{\text {sep }}\left(\mathbb{Z}^{d}\right)$ and thus also

$$
\lim _{t \downarrow 0} \mathbb{E}_{\mathbf{u}}\left[\mathbf{u}_{t}^{(m)}(\mathbf{x})\right]=0=\int_{\mathcal{M}_{K}^{\mathrm{sep}}\left(\mathbb{Z}^{d}\right)} \tilde{\mathbf{u}}^{(m)}(\mathbf{x}) J(\mathbf{u})(d \tilde{\mathbf{u}}) .
$$

Therefore, we now assume in addition that the coloring $m$ is constant on each block of $\pi$. Then we can also define a coloring $c \in\{1,2\}^{k}$ by

$$
c_{j}:=m_{i} \quad \text { for } i \in \pi_{j}, j=1, \ldots, k .
$$

Observe that for all $\tilde{\mathbf{u}}=\left(\tilde{u}^{(1)}, \tilde{u}^{(2)}\right) \in \mathcal{M}_{K}^{\text {sep }}\left(\mathbb{Z}^{d}\right)$, we have $\tilde{u}^{(i)}(\cdot)=\left(\tilde{u}^{(1)}(\cdot)+\right.$ $\left.\tilde{u}^{(2)}(\cdot)\right) \mathbb{1}_{\left\{\tilde{u}^{(i)}(\cdot)>0\right\}}$ and thus

$$
\tilde{\mathbf{u}}^{(m)}(\mathbf{x})=\prod_{j=1}^{k}\left(\tilde{u}^{\left(c_{j}\right)}\left(y_{j}\right)\right)^{\left|\pi_{j}\right|}=\tilde{\mathbf{u}}^{(c)}(\mathbf{y}) \prod_{j=1}^{k}\left(\tilde{u}^{(1)}\left(y_{j}\right)+\tilde{u}^{(2)}\left(y_{j}\right)\right)^{\left|\pi_{j}\right|-1} .
$$

Using this representation for $\tilde{\mathbf{u}}=\mathbf{u}_{t} \in \mathcal{M}_{K}^{\text {sep }}\left(\mathbb{Z}^{d}\right)$ with $t>0$ and taking the expectation, we get

$$
\mathbb{E}_{\mathbf{u}}\left[\mathbf{u}_{t}^{(m)}(\mathbf{x})\right]=\mathbb{E}_{\mathbf{u}}\left[\mathbf{u}_{t}^{(c)}(\mathbf{y})\right] \prod_{j=1}^{k} w_{t}\left(y_{j}\right)^{\left|\pi_{j}\right|-1}, \quad t>0
$$


since the sum $u_{t}^{(1)}+u_{t}^{(2)}=w_{t}=S_{t}\left(u^{(1)}+u^{(2)}\right)$ is deterministic. Applying the moment duality (6) to the expectation on the RHS, we obtain by dominated convergence that

$$
\mathbb{E}_{\mathbf{u}}\left[\mathbf{u}_{t}^{(c)}(\mathbf{y})\right]=\mathbb{E}_{\mathbf{y}}\left[\sum_{b \in\{1,2\}^{k}} \mathbf{u}^{(b)}\left(X_{t}\right) M_{t}^{[\infty]}\left(X, \delta_{c}\right)(b)\right] \stackrel{t \downarrow 0}{\longrightarrow} \mathbf{u}^{(c)}(\mathbf{y}),
$$

where we use that by Proposition 4.5, under $\mathbb{P}_{\mathbf{y}}$ we have $\lim _{t \downarrow 0} M_{t}^{[\infty]}\left(X, \delta_{c}\right)=$ $M_{0}^{[\infty]}\left(X, \delta_{c}\right)=\delta_{c}$ since $\pi\left(X_{0}\right)$ is the partition into singletons (note that the components of $\mathbf{y}$ are all distinct). Substituting this into (38), we get

$$
\mathbb{E}_{\mathbf{u}}\left[\mathbf{u}_{t}^{(m)}(\mathbf{x})\right] \rightarrow \mathbf{u}^{(c)}(\mathbf{y}) \prod_{j=1}^{k}\left(u^{(1)}\left(y_{j}\right)+u^{(2)}\left(y_{j}\right)\right)^{\left|\pi_{j}\right|-1} \quad \text { as } t \downarrow 0 .
$$

Using the definition (8) of the function $J$, it is a straightforward calculation to check that the RHS of the previous display coincides with the integral $\int_{\mathcal{M}_{K}^{\text {sep }}\left(\mathbb{Z}^{d}\right)} \tilde{\mathbf{u}}^{(m)}(\mathbf{x}) J(\mathbf{u})(d \tilde{u})$.

ProOF OF Proposition 5.3. Recall that in both continuous and discrete space, we have defined $\left(P_{t}\right)_{t \geq 0}$ as the family of probability laws (30).

(1) In the first step, fix $t>0$ and let $\mathcal{H} \subseteq \mathcal{C}\left(\mathcal{M}_{K}(\mathcal{S})\right)$ denote the system of all functions $f_{\phi, m}$ of the form (33) with $\phi=\bigotimes_{i=1}^{n} \phi_{i}$, where $\phi_{i} \in \mathcal{C}_{c}(\mathcal{S}), i=$ $1, \ldots, n$. Note that $\mathcal{H}$ is closed under multiplication and separates the points of $\mathcal{M}_{K}(\mathcal{S})$. Writing $\mathcal{A} \subseteq \mathcal{C}\left(\mathcal{M}_{K}(\mathcal{S})\right)$ for the algebra generated by $\mathcal{H}$ and the constant functions, we conclude using Lemma 5.4 that for all $f \in \mathcal{A}$ we have $P_{t}^{[\gamma]} f \in$ $\mathcal{C}\left(\mathcal{M}_{K}(\mathcal{S})\right), P_{t} f \in \mathcal{C}\left(\mathcal{M}_{K}(\mathcal{S})\right)$ and (32) holds. Since by the Stone-Weierstrass theorem $\mathcal{A}$ is dense in $\mathcal{C}\left(\mathcal{M}_{K}(\mathcal{S})\right)$ w.r.t. the uniform norm [recall that $\mathcal{M}_{K}(\mathcal{S})$ is compact], this extends easily to all $f \in \mathcal{C}\left(\mathcal{M}_{K}(\mathcal{S})\right)$. We have thus shown that the strong convergence (32) holds for all $f \in \mathcal{C}\left(\mathcal{M}_{K}(\mathcal{S})\right)$ and that

$$
P_{t}\left(\mathcal{C}\left(\mathcal{M}_{K}(\mathcal{S})\right)\right) \subseteq \mathcal{C}\left(\mathcal{M}_{K}(\mathcal{S})\right) \quad \text { and } \quad P_{t}^{[\gamma]}\left(\mathcal{C}\left(\mathcal{M}_{K}(\mathcal{S})\right)\right) \subseteq \mathcal{C}\left(\mathcal{M}_{K}(\mathcal{S})\right) .
$$

(2) By the continuity of the paths of $\left(\mathbf{u}_{t}^{[\gamma]}\right)_{t \geq 0}$, we have $P_{t}^{[\gamma]} f(\mathbf{u}) \rightarrow f(\mathbf{u})$ as $t \downarrow 0$, for all $f \in \mathcal{C}\left(\mathcal{M}_{K}(\mathcal{S})\right)$ and $\mathbf{u} \in \mathcal{M}_{K}(\mathcal{S})$. It is well known that together with the second inclusion in (39), this implies already the strong continuity of $\left(P_{t}^{[\gamma]}\right)_{t \geq 0}$ on $\mathcal{C}\left(\mathcal{M}_{K}(\mathcal{S})\right)$, that is, it is a Feller semigroup. This proves part (a) of the proposition.

(3) By a monotone class argument, the first inclusion in (39) implies in particular that $P_{t} f$ is measurable for each bounded measurable $f: \mathcal{M}_{K}(\mathcal{S}) \rightarrow \mathbb{R}$, that is, $P_{t}(\cdot, \cdot)$ as defined in (30) is indeed a transition kernel on $\mathcal{M}_{K}(\mathcal{S})$ for each $t \geq 0$. The semigroup property of this family of kernels follows easily from the strong convergence (32), which has already been shown in step (1) above, together with 
the semigroup property of $\left(P_{t}^{[\gamma]}\right)_{t \geq 0}$, since

$$
\begin{aligned}
\left|P_{t+s} f(\mathbf{u})-P_{t} P_{s} f(\mathbf{u})\right| & =\lim _{\gamma \rightarrow \infty}\left|P_{t+s}^{[\gamma]} f(\mathbf{u})-P_{t}^{[\gamma]} P_{s} f(\mathbf{u})\right| \\
& =\lim _{\gamma \rightarrow \infty}\left|P_{t}^{[\gamma]}\left(P_{s}^{[\gamma]} f-P_{s} f\right)(\mathbf{u})\right| \\
& \leq \lim _{\gamma \rightarrow \infty}\left\|P_{s}^{[\gamma]} f-P_{s} f\right\|_{\infty}=0
\end{aligned}
$$

for all $f \in \mathcal{C}\left(\mathcal{M}_{K}(\mathcal{S})\right), \mathbf{u} \in \mathcal{M}_{K}(\mathcal{S})$ [note that $P_{s} f$ is continuous by (39)]. This completes the proof of part (b) of the proposition.

(4) For part (c), we now show that in the continuous case $\mathcal{S}=\mathbb{R}$ we have

$$
P_{t} f(\mathbf{u}) \rightarrow f(\mathbf{u}) \quad \text { as } t \downarrow 0
$$

for all $f \in \mathcal{C}\left(\mathcal{M}_{K}(\mathbb{R})\right)$ and $\mathbf{u} \in \mathcal{M}_{K}(\mathbb{R})$. Again, together with the first inclusion in (39) this implies the strong continuity of $\left(P_{t}\right)_{t \geq 0}$ on $\mathcal{C}\left(\mathcal{M}_{K}(\mathbb{R})\right)$ and shows that it is a Feller semigroup. We stress that (40) does not simply follow from the fact that the Meyer-Zheng limit point $\left(\mathbf{u}_{t}\right)_{t \geq 0}$, from whose law the definition of $\left(P_{t}\right)_{t \geq 0}$ is derived, has càdlàg paths (see Remark 5.2). However, we know by Lemma 5.4(b) that (40) holds for functions $f=f_{\phi, m}$ of the form (33). By another Stone-Weierstrass argument as in step (1) above, this is easily extended to all $f \in \mathcal{C}\left(\mathcal{M}_{K}(\mathbb{R})\right)$. This concludes the proof of Proposition 5.3 for the continuous case.

(5) For the discrete case $\mathcal{S}=\mathbb{Z}^{d}$, it remains to show part (d). Note that since $P_{t}(\mathbf{u}, \cdot)$ is concentrated on $\mathcal{M}_{K}^{\mathrm{sep}}\left(\mathbb{Z}^{d}\right)$ for $t>0$, the semigroup restricts in a canonical way to the (compact) subspace $\mathcal{M}_{K}^{\text {sep }}\left(\mathbb{Z}^{d}\right)$. But on this subspace, we know by Lemma 5.4(c) that the convergence (40) holds for functions $f=f_{\phi, m}$ of the form (33) and all $\mathbf{u} \in \mathcal{M}_{K}^{\text {sep }}\left(\mathbb{Z}^{d}\right)$. Thus, we can argue analogously to step (4) above to conclude that $\left(P_{t}\right)_{t \geq 0}$ is a Feller semigroup on $\mathcal{M}_{K}^{\mathrm{sep}}\left(\mathbb{Z}^{d}\right)$.

Finally, if $\mathbf{u} \in \mathcal{M}_{K}\left(\mathbb{Z}^{d}\right)$ is such that $u^{(1)}(x) u^{(2)}(x) \neq 0$ for some $x \in \mathbb{Z}^{d}$, then we get for the mixed second moment that

$$
\lim _{t \downarrow 0} \mathbb{E}_{\mathbf{u}}\left[u_{t}^{(1)}(x) u_{t}^{(2)}(x)\right]=0 \neq u^{(1)}(x) u^{(2)}(x),
$$

since the separation of types holds at each positive time $t>0$. This shows that the semigroup $\left(P_{t}\right)_{t \geq 0}$ is not right-continuous at $t=0$ if we consider it on the big space $\mathcal{M}_{K}\left(\mathbb{Z}^{d}\right)$.

Now it is straightforward to finish the proof of Theorem 2.8:

Proof of TheOREM 2.8. Consider first the continuous case $\mathcal{S}=\mathbb{R}$ : Let $\mathbf{u}_{0} \in \mathcal{M}_{K}(\mathbb{R})$ with $K>0$. By standard theory (see, e.g., [11], Theorem 4.2.5), the strong convergence (32) of the Feller semigroups on $\mathcal{C}\left(\mathcal{M}_{K}(\mathbb{R})\right)$ implies weak convergence of the family of processes $\left(\mathbf{u}_{t}^{[\gamma]}\right)_{t \geq 0}$ as $\gamma \uparrow \infty$ in $D_{[0, \infty)}\left(\mathcal{M}_{K}(\mathbb{R})\right)$ w.r.t. the Skorokhod topology, and the unique limit $\left(\mathbf{u}_{t}\right)_{t \geq 0}$ is a Markov Feller 
process with semigroup $\left(P_{t}\right)_{t \geq 0}$. Since the approximating processes $\left(\mathbf{u}_{t}^{[\gamma]}\right)_{t \geq 0}$ are continuous and the Skorokhod topology coincides with the uniform topology on compacts on $\mathcal{C}_{[0, \infty)}\left(\mathcal{M}_{K}(\mathbb{R})\right)$, which is closed in $D_{[0, \infty)}\left(\mathcal{M}_{K}(\mathbb{R})\right)$, the limit is in fact in $\mathcal{C}_{[0, \infty)}\left(\mathcal{M}_{K}(\mathbb{R})\right)$.

For the discrete case $\mathcal{S}=\mathbb{Z}^{d}$, we do not obtain convergence in the Skorokhod topology, and indeed the assumptions of [11], Theorem 4.2.5, are not fulfilled: Namely, the limiting semigroup $\left(P_{t}\right)_{t \geq 0}$ is not Feller on the "big" space $\mathcal{M}_{K}\left(\mathbb{Z}^{d}\right)$ where the approximating semigroups $\left(P_{t}^{[\gamma]}\right)_{t \geq 0}$ live. However, we can still argue along the lines of the proof of [11], Theorem 4.2.5, to obtain the Markov property: Fix $\mathbf{u} \in \mathcal{M}_{K}\left(\mathbb{Z}^{d}\right)$. Consider $m \in \mathbb{N}, 0 \leq t_{1}<t_{2}<\cdots<t_{m}<t_{m+1}$ and $f_{i} \in \mathcal{C}\left(\mathcal{M}_{K}\left(\mathbb{Z}^{d}\right)\right), i=1, \ldots, m+1$. Recall that $\left(\mathbf{u}_{t}\right)_{t \geq 0} \in D_{[0, \infty)}\left(\mathcal{M}_{K}\left(\mathbb{Z}^{d}\right)\right)$ denotes a limit point w.r.t. the Meyer-Zheng-topology of the tight family of processes $\left\{\left(\mathbf{u}_{t}^{[\gamma]}\right)_{t \geq 0}: \gamma>0\right\}$. Again using [20], Theorem 1.1, Corollary 1.4, we may assume that there is a sequence $\gamma_{k} \uparrow \infty$ and a subset $I \subseteq \mathbb{R}^{+}$of full Lebesgue measure such that for all $t \in I$ we have $\mathbf{u}_{t}^{\left[\gamma_{k}\right]} \rightarrow \mathbf{u}_{t}$ as $k \rightarrow \infty$, almost surely. If we suppose in addition that $t_{i} \in I$, we can use dominated convergence, the Markov property of $\left(\mathbf{u}_{t}^{[\gamma]}\right)_{t \geq 0}$ and Proposition 5.3 to obtain

$$
\begin{aligned}
\mathbb{E}_{\mathbf{u}}\left[\prod_{i=1}^{m+1} f_{i}\left(\mathbf{u}_{t_{i}}\right)\right] & =\lim _{k \uparrow \infty} \mathbb{E}_{\mathbf{u}}\left[\prod_{i=1}^{m+1} f_{i}\left(\mathbf{u}_{t_{i}}^{\left[\gamma_{k}\right]}\right)\right] \\
& =\lim _{k \uparrow \infty} \mathbb{E}_{\mathbf{u}}\left[\prod_{i=1}^{m} f_{i}\left(\mathbf{u}_{t_{i}}^{\left[\gamma_{k}\right]}\right) P_{t_{m+1}-t_{m}}^{\left[\gamma_{k}\right]} f_{m+1}\left(\mathbf{u}_{t_{m}}^{\left[\gamma_{k}\right]}\right)\right] \\
& =\mathbb{E}_{\mathbf{u}}\left[\prod_{i=1}^{m} f_{i}\left(\mathbf{u}_{t_{i}}\right) P_{t_{m+1}-t_{m}} f_{m+1}\left(\mathbf{u}_{t_{m}}\right)\right]
\end{aligned}
$$

[note that $P_{t_{m+1}-t_{m}} f_{m+1}$ is continuous by Proposition 5.3(b)]. Using the rightcontinuity of the paths of $\left(\mathbf{u}_{t}\right)_{t \geq 0}$ and of the semigroup $\left(P_{t}\right)_{t \geq 0}$ for $t \in(0, \infty)$ [see Proposition 5.3(d)], this identity then holds also without the restriction that $t_{i} \in I$. By induction, this shows that the finite-dimensional distributions of $\left(\mathbf{u}_{t}\right)_{t \geq 0} \in$ $D_{[0, \infty)}\left(\mathcal{M}_{K}\left(\mathbb{Z}^{d}\right)\right)$ are uniquely determined, thus it is the unique limit point of $\left\{\left(\mathbf{u}_{t}^{[\gamma]}\right)_{t \geq 0}: \gamma>0\right\}$ w.r.t. the Meyer-Zheng topology. Moreover, (41) identifies the limit $\left(\mathbf{u}_{t}\right)_{t \geq 0}$ as Markov process with transition semigroup $\left(P_{t}\right)_{t \geq 0}$, starting from the (possibly random) initial distribution $\mathcal{L}\left(\lim _{t \downarrow 0} \mathbf{u}_{t} \mid \mathbb{P}_{\mathbf{u}}\right)$ on $\mathcal{M}_{K}^{\text {sep }}\left(\mathbb{Z}^{d}\right)$. In view of Lemma 5.4(c), this initial distribution coincides with $J$ (u) from (8). This concludes the proof of Theorem 2.8 .

5.2. Some notation and preliminaries. We continue to use all the notation from Section 2.2, in particular those introduced after Remark 2.9. Moreover, we write $\mathbb{R}^{n, \uparrow}:=\left\{\mathbf{x} \in \mathbb{R}^{n}: x_{1}<x_{2}<\cdots<x_{n}\right\}$ for the space of increasing sequences of length $n$ in $\mathbb{R}$, and analogously $\mathbb{Q}^{n, \uparrow}$ or $[a, b]^{n, \uparrow}$ for sequences in $\mathbb{Q}$ or $[a, b]$. 
LEMMA 5.5. Let $\mathbf{u}=\left(u^{(1)}, u^{(2)}\right) \in \mathcal{U}$.

(a) Suppose that $a<b$ with $a \in \operatorname{supp}\left(u^{(i)}\right)$ and $b \in \operatorname{supp}\left(u^{(3-i)}\right), i \in\{1,2\}$. Then there must be an interface point between $a$ and $b$, that is, $\mathcal{I}(\mathbf{u}) \cap[a, b] \neq \varnothing$.

(b) Suppose that $a, b \in \mathcal{I}(\mathbf{u})$ with $a<b$ and that there are no interface points (strictly) between $a$ and $b$, that is, $\mathcal{I}(\mathbf{u}) \cap(a, b)=\varnothing$. Then there exists $i \in\{1,2\}$ with $(a, b) \subseteq \operatorname{supp}\left(u^{(i)}\right) \backslash \operatorname{supp}\left(u^{(3-i)}\right)$.

Proof. (a) Set $A_{i}:=\operatorname{supp}\left(u^{(i)}\right) \cap[a, b], i=1,2$, which are closed sets and by assumption non-empty. Since $u \in \mathcal{U}$, we have $\operatorname{supp}\left(u^{(1)}\right) \cup \operatorname{supp}\left(u^{(2)}\right)=$ $\operatorname{supp}\left(u^{(1)}+u^{(2)}\right)=\mathbb{R}$. Then the fact that $A_{1} \cup A_{2}=[a, b]$ is connected implies that the intersection $A_{1} \cap A_{2}=\mathcal{I}(\mathbf{u}) \cap[a, b]$ is non-empty.

(b) Set $B_{i}:=(a, b) \backslash \operatorname{supp}\left(u^{(i)}\right), i=1,2$, which are open sets and also disjoint since $\operatorname{supp}\left(u^{(1)}\right) \cup \operatorname{supp}\left(u^{(2)}\right)=\mathbb{R}$. From $\mathcal{I}(\mathbf{u}) \cap(a, b)=\varnothing$, it follows that $B_{1} \cup$ $B_{2}=(a, b)$, which is connected. This implies that either $B_{1}$ or $B_{2}$ is empty, and hence the other equals $(a, b)$.

LEMMA 5.6. Let $\mathbf{u} \in \mathcal{U}$ and $n \in \mathbb{N}$. The following are equivalent:

(a) $\mathbf{u} \in \bigcup_{k=0}^{n} \mathcal{U}_{k}$.

(b) For each $a \in \mathbb{Q}^{2(n+2), \uparrow}$ and alternating coloring $m \in\{1,2\}^{n+2}$, we have

$$
\prod_{i=1}^{n+2} u^{\left(m_{i}\right)}\left(\left[a_{2 i-1}, a_{2 i}\right]\right)=0 .
$$

(c) For each $m \in\{1,2\}^{n+2}$ alternating, we have $\mathbf{u}^{(m)}(\mathbf{x})=0$ for Lebesgue-a.e. $\mathbf{x} \in \mathbb{R}^{n+2, \uparrow}$.

Proof. $\quad$ (a) $\Rightarrow$ (b): Suppose that for some $a \in \mathbb{Q}^{2(n+2), \uparrow}$ and alternating configuration $m$, we have $\prod_{i=1}^{n+2} u^{\left(m_{i}\right)}\left(\left[a_{2 i-1}, a_{2 i}\right]\right)>0$. Since $u^{(1)}, u^{(2)}$ are absolutely continuous, for each $i=1, \ldots, n+2$ we can pick two distinct points $x_{2 i-1}<x_{2 i}$ in $\left(a_{2 i-1}, a_{2 i}\right) \cap \operatorname{supp}\left(u^{\left(m_{i}\right)}\right) \neq \varnothing$ such that $\mathbf{x} \in \mathbb{R}^{2(n+2), \uparrow}$. Then we have $x_{2 i}<x_{2 i+1}$, $x_{2 i} \in \operatorname{supp}\left(u^{\left(m_{i}\right)}\right)$ and $x_{2 i+1} \in \operatorname{supp}\left(u^{\left(m_{i+1}\right)}\right)$ for all $i=1, \ldots,(n+2)$. Since $m_{i} \neq m_{i+1}$, by Lemma 5.5 there must be an interface point in each of the disjoint intervals $\left[x_{2 i}, x_{2 i+1}\right], i=1, \ldots, n+1$, so that $\mathbf{u} \notin \bigcup_{k=0}^{n} \mathcal{U}_{k}$.

(b) $\Rightarrow$ (a): Suppose that $\mathbf{u} \notin \bigcup_{k=0}^{n} \mathcal{U}_{k}$, that is, there are at least $n+1$ interfaces $x_{1}<x_{2}<\cdots<x_{n+1}$. Let $\delta:=\frac{1}{2} \min \left\{x_{i+1}-x_{i}: 1 \leq i \leq n\right\}$. Since $x_{1}$ is an interface point and $u^{(1)}+u^{(2)}$ is equivalent to Lebesgue measure, there must be a pair of indices $(k, \ell) \in\{(1,2),(2,1)\}$ such that $u^{(k)}\left(\left[x_{1}-\delta, x_{1}\right]\right)>0$ and $u^{(\ell)}\left(\left[x_{1}, x_{1}+\delta\right]\right)>0$. Let $m \in\{1,2\}^{n+2}$ be the alternating coloring with $m_{1}=k$. Using the fact that $u^{(j)}\left(\left[x_{i}-\delta, x_{i}+\delta\right]\right)>0$ for all $i=2, \ldots, n+1$ and $j=1,2$, we can set $a_{1}^{\prime}:=x_{1}-\delta, a_{2}^{\prime}:=a_{3}^{\prime}:=x_{1}, a_{4}^{\prime}:=x_{1}+\delta$ and $a_{2 i+1}^{\prime}:=x_{i}-\delta$, $a_{2 i+2}^{\prime}:=x_{i}+\delta$ for $i=2, \ldots, n+1$ in order to obtain $u^{\left(m_{i}\right)}\left(\left[a_{2 i-1}^{\prime}, a_{2 i}^{\prime}\right]\right)>0$ 
for all $i=1, \ldots, n+2$. Now we can choose $a \in \mathbb{Q}^{2(n+2), \uparrow}$ so that $\left[a_{2 i-1}, a_{2 i}\right] \subset$ $\left(a_{2 i-1}^{\prime}, a_{2 i}^{\prime}\right)$ and $u^{\left(m_{i}\right)}\left(\left[a_{2 i-1}, a_{2 i}\right]\right)>0, i=1, \ldots, n+2$, as required.

(b) $\Leftrightarrow$ (c): By continuity of measures and the fact that $\mathbb{Q}$ is dense in $\mathbb{R}$, under (b) equation (42) holds in fact for all $a \in \mathbb{R}^{2(n+2), \uparrow}$. The equivalence then follows from the absolute continuity of the measures.

5.3. Non-proliferation of interfaces. In this section, we prove that the number of interfaces is non-increasing. Throughout this and the remaining sections, $\left(\mathbf{u}_{t}\right)_{t \geq 0}$ will denote the continuous-space infinite rate symbiotic branching process $\operatorname{cSBM}(-1, \infty)$ from Theorem 2.8(a).

THEOREM 5.7. Suppose that $\mathbf{u}_{0} \in \mathcal{U}_{n}$. Then,

$$
\mathbb{P}_{\mathbf{u}_{0}}\left(\mathbf{u}_{t} \in \bigcup_{k=0}^{n} \mathcal{U}_{k} \text { for all } t \geq 0\right)=1 \text {. }
$$

The key step to prove the theorem is the following lemma, for which we recall the notation $f_{\phi, m}$ from (33).

LEMMA 5.8. Let $n \in \mathbb{N}_{0}$. Then for all $\mathbf{u}_{0} \in \mathcal{M}_{b}(\mathbb{R})^{2}$, each alternating coloring $m \in\{1,2\}^{n+2}$ and $\phi \in L^{1}\left(\mathbb{R}^{n+2}\right)$ with $\operatorname{supp}(\phi) \subseteq \mathbb{R}^{n+2, \uparrow}$, we have

$$
P_{t} f_{\phi, m}\left(\mathbf{u}_{0}\right)=\mathbb{E}_{\mathbf{u}_{0}}\left[f_{\phi, m}\left(\mathbf{u}_{t}\right)\right]=\int_{\mathbb{R}^{n+2, \uparrow}} \phi(\mathbf{x}) \mathbb{E}_{\mathbf{x}}\left[\mathbf{u}_{0}^{(m)}\left(X_{t}\right) \mathbb{1}_{t<\tau}\right] d \mathbf{x}
$$

where

$$
\tau:=\inf \left\{t \geq 0: \exists 1 \leq i<j \leq n \text { with } X_{t}^{(i)}=X_{t}^{(j)}\right\}
$$

is the first collision time of the Brownian motions $\left(X_{t}\right)_{t \geq 0}$. If in addition $\mathbf{u}_{0} \in \mathcal{U}_{n}$, we have

$$
P_{t} f_{\phi, m}\left(\mathbf{u}_{0}\right)=0
$$

Proof. Let $n \in \mathbb{N}_{0}$ and $m \in\{1,2\}^{n+2}$ alternating. For $\mathbf{x} \in \mathbb{R}^{n+2, \uparrow}$, denote by $(i, i+1)$ the index pair of the two Brownian motions involved in the collision at time $\tau$, starting from $\mathbf{x}$. Then $M_{t}^{[\infty]}\left(X, \delta_{m}\right)=M_{0}^{[\infty]}\left(X, \delta_{m}\right)=\delta_{m}$ for $t \leq \tau$, and $\pi\left(X_{\tau}\right)=\{\{1\},\{2\}, \ldots,\{i-1\},\{i, i+1\},\{i+2\}, \ldots,\{n+2\}\}$. Since $m$ is alternating, we have $m_{i} \neq m_{i+1}$, hence by the explicit form of $K_{\infty}$ (see Proposition 4.2)

$$
K_{\infty}\left(M_{\tau}^{[\infty]}, \pi\left(X_{\tau}\right)\right) \equiv 0
$$

In view of the recursive definition of $M_{t}^{[\infty]}$ (see Proposition 4.6), this implies $M_{t}^{[\infty]}\left(X, \delta_{m}\right) \equiv 0$ for all $t>\tau$, thus we have

$$
M_{t}^{[\infty]}\left(X, \delta_{m}\right)=\mathbb{1}_{t \leq \tau} \delta_{m}
$$


By the moment duality (6), since $\operatorname{supp}(\phi) \subseteq \mathbb{R}^{n+2, \uparrow}$ this implies

$$
\begin{aligned}
\mathbb{E}_{\mathbf{u}_{0}}\left[f_{\phi, m}\left(\mathbf{u}_{t}\right)\right] & =\int_{\mathbb{R}^{n+2}} \phi(\mathbf{x}) \mathbb{E}_{\mathbf{x}}\left[\sum_{b \in\{1,2\}^{n+2}} M_{t}^{[\infty]}\left(X, \delta_{m}\right)(b) \mathbf{u}_{0}^{(b)}\left(X_{t}\right)\right] d \mathbf{x} \\
& =\int_{\mathbb{R}^{n+2, \uparrow}} \phi(\mathbf{x}) \mathbb{E}_{\mathbf{x}}\left[\mathbf{u}_{0}^{(m)}\left(X_{t}\right) \mathbb{1}_{t<\tau}\right] d \mathbf{x},
\end{aligned}
$$

where we also used that $\mathbb{P}_{\mathbf{x}}(\tau=t)=0$ for each fixed $t>0$. Thus, (43) is proved.

Moreover, if in addition $\mathbf{u}_{0} \in \mathcal{U}_{n}$, then by Lemma 5.6 we have $\mathbf{u}_{0}^{(m)}(\mathbf{x})=0$ for almost every $\mathbf{x} \in \mathbb{R}^{n+2, \uparrow}$, and under $\mathbb{P}_{\mathbf{x}}$ we have $X_{t} \in \mathbb{R}^{n+2, \uparrow}$ a.s. on the event $\{t<\tau\}$. Thus in this case the integrand on the RHS of the previous display is zero, and (44) is established.

Proof of THEOREM 5.7. Fix $t \geq 0$, an increasing vector $a \in \mathbb{R}^{2(n+2), \uparrow}$ and an alternating configuration $m \in\{1,2\}^{n+2}$. Since $\mathbf{u}_{0} \in \mathcal{U}_{n}$, by Lemma 5.8 applied to the function $\phi(\mathbf{x}):=\prod_{i=1}^{n+2} \mathbb{1}_{\left[a_{2 i-1}, a_{2 i}\right]}\left(x_{i}\right)$ with $\operatorname{supp}(\phi) \subseteq \mathbb{R}^{n+2, \uparrow}$ we obtain

$$
\mathbb{E}_{\mathbf{u}_{0}}\left[\prod_{i=1}^{n+2} u_{t}^{\left(m_{i}\right)}\left(\left[a_{2 i-1}, a_{2 i}\right]\right)\right]=\mathbb{E}_{\mathbf{u}_{0}}\left[\left\langle\mathbf{u}_{t}^{(m)}, \phi\right\rangle\right]=0
$$

and consequently $\prod_{i=1}^{n+2} u_{t}^{\left(m_{i}\right)}\left(\left[a_{2 i-1}, a_{2 i}\right]\right)=0, \mathbb{P}_{\mathbf{u}_{0}}$-a.s. Now denote by $m^{(j)} \in$ $\{1,2\}^{n+2}$ the alternating coloring starting with $m_{1}^{(j)}=j$ for $j=1,2$. Using Lemma 5.6, for each fixed $t \geq 0$ we have

$$
\left\{\mathbf{u}_{t} \in \bigcup_{k=0}^{n} \mathcal{U}_{k}\right\}=\bigcap_{j \in\{1,2\}} \bigcap_{a \in \mathbb{Q}^{2(n+2), \uparrow}}\left\{\prod_{i=1}^{n+2} u_{t}^{\left(m_{i}^{(j)}\right)}\left(\left[a_{2 i-1}, a_{2 i}\right]\right)=0\right\},
$$

which is a countable intersection of events of probability 1 . As a consequence, we get $\mathbb{P}_{\mathbf{u}_{0}}\left(\mathbf{u}_{t} \in \bigcup_{k=0}^{n} \mathcal{U}_{k}\right)=1$ for each $t \geq 0$, and hence also

$$
\mathbb{P}_{\mathbf{u}_{0}}\left(\mathbf{u}_{t} \in \bigcup_{k=0}^{n} \mathcal{U}_{k} \text { for all } t \in[0, \infty) \cap \mathbb{Q}\right)=1
$$

To extend this result to all $t \geq 0$, we use the fact that $\left(\mathbf{u}_{t}\right)_{t \geq 0}$ has rightcontinuous paths and hence we have that $\lim _{s \downarrow t} \prod_{i=1}^{n+2} u_{s}^{\left(m_{i}^{(j)}\right)}\left(\left[a_{2 i-1}, a_{2 i}\right]\right)=$ $\prod_{i=1}^{n} u_{t}^{\left(m_{i}^{(j)}\right)}\left(\left[a_{2 i-1}, a_{2 i}\right]\right)$, which shows that

$$
\begin{aligned}
\left\{\mathbf{u}_{t}\right. & \left.\in \bigcup_{k=0}^{n} \mathcal{U}_{k} \text { for all } t \in[0, \infty) \cap \mathbb{Q}\right\} \\
& =\bigcap_{t \in[0, \infty) \cap \mathbb{Q}} \bigcap_{j \in\{1,2\}} \bigcap_{a \in \mathbb{Q}^{2(n+2), \uparrow}}\left\{\prod_{i=1}^{n+2} u_{t}^{\left(m_{i}^{(j)}\right)}\left(\left[a_{2 i-1}, a_{2 i}\right]\right)=0\right\}
\end{aligned}
$$




$$
\begin{aligned}
& =\bigcap_{t \in[0, \infty)} \bigcap_{j \in\{1,2\}} \bigcap_{a \in \mathbb{Q}^{2(n+2), \uparrow}}\left\{\prod_{i=1}^{n+2} u_{t}^{\left(m_{i}^{(j)}\right)}\left(\left[a_{2 i-1}, a_{2 i}\right]\right)=0\right\} \\
& =\left\{\mathbf{u}_{t} \in \bigcup_{k=0}^{n} \mathcal{U}_{k} \text { for all } t \geq 0\right\} .
\end{aligned}
$$

Hence, we have shown that almost surely, there are at most $n$ interfaces at any one time.

5.4. Movement of a single interface. In this section, we will prove Theorem 2.10, showing that a single interface moves according to (9). Again the key point is that the sum evolves as the solution to the deterministic heat equation, that is, $u_{t}^{(1)}+u_{t}^{(2)}=w_{t}=S_{t} w_{0}$. In particular, recalling the definition of $m\left(\mathbf{u}_{t}, x\right)$ from Section 2.2, we have

$$
u_{t}^{(i)}(d x)=\mathbb{1}_{\left\{m\left(\mathbf{u}_{t}, x\right)=i\right\}} w_{t}(x) d x, \quad i=1,2,
$$

a fact we will use repeatedly.

LEMma 5.9. Assume $\mathbf{u}_{0} \in \mathcal{U}_{n}$ for some $n \in \mathbb{N}$. Then for each fixed $t>0$ we have $\lim _{x \rightarrow \pm \infty} m\left(\mathbf{u}_{t}, x\right)=\lim _{x \rightarrow \pm \infty} m\left(\mathbf{u}_{0}, x\right)$ almost surely.

Proof. Fix $t>0$. First, observe that since by Theorem 5.7 there is a finite number of interfaces at time $t$, the $\operatorname{limits}_{x \rightarrow \pm \infty} m\left(\mathbf{u}_{t}, x\right)$ exist in $\{1,2\}$.

Let $\phi \in \mathcal{C}_{c}(\mathbb{R})$. Since $w_{t}$ is deterministic, we can use (45) and the moment duality to obtain

$$
\begin{aligned}
\mathbb{E}_{\mathbf{u}_{0}}\left[\int_{\mathbb{R}} \phi(x) \mathbb{1}_{m\left(\mathbf{u}_{t}, x\right)=i} d x\right] & =\mathbb{E}_{\mathbf{u}_{0}}\left[\left\langle\frac{\phi}{w_{t}}, u_{t}^{(i)}\right\rangle\right] \\
& =\left\langle\frac{\phi}{w_{t}}, S_{t} u_{0}^{(i)}\right\rangle \\
& =\left\langle\phi,\left(1+\frac{S_{t} u_{0}^{(3-i)}}{S_{t} u_{0}^{(i)}}\right)^{-1}\right\rangle, \quad i=1,2 .
\end{aligned}
$$

Now assume w.l.o.g. that $\lim _{x \rightarrow-\infty} m\left(\mathbf{u}_{0}, x\right)=1$ and $\min \mathcal{I}\left(\mathbf{u}_{0}\right)=0$. Then we have $S_{t} u_{0}^{(2)}(x) \leq\left\|u_{0}^{(2)}\right\|_{\infty} \int_{0}^{\infty}(2 \pi t)^{-\frac{1}{2}} e^{-(y-x)^{2} / 2 t} d y$, and since $u_{0}^{(1)}([-2 \delta$, $-\delta])>0$ for $\delta>0$, we have for $x<-2 \delta$

$$
S_{t} u_{0}^{(1)}(x) \geq u_{0}^{(1)}([-2 \delta,-\delta])(2 \pi t)^{-\frac{1}{2}} e^{-(-\delta-x)^{2} / 2 t} .
$$

Therefore, for some $C=C(\mathbf{u}, \delta)>0$

$$
0 \leq \frac{S_{t} u_{0}^{(2)}(x)}{S_{t} u_{0}^{(1)}(x)} \leq C \int_{0}^{\infty} e^{\left(-y^{2}+2 y x+2 \delta x\right) / 2 t} d y \leq C e^{\delta x / t} \int_{0}^{\infty} e^{-y^{2} / 2 t} d y,
$$


which converges to 0 as $x \rightarrow-\infty$. Thus

$$
\left(1+\frac{S_{t} u_{0}^{(2)}(x)}{S_{t} u_{0}^{(1)}(x)}\right)^{-1} \stackrel{x \rightarrow-\infty}{\longrightarrow} 1
$$

Now choose $\phi \in \mathcal{C}_{c}(\mathbb{R})$ nonnegative with $\int_{\mathbb{R}} \phi(x) d x=1$ and define $\phi_{n}(\cdot):=\phi(\cdot+$ $n), n \in \mathbb{N}$. Then (46) and (47) show that

$$
\mathbb{E}_{\mathbf{u}_{0}}\left[\int_{\mathbb{R}} \phi_{n}(x) \mathbb{1}_{m\left(\mathbf{u}_{t}, x\right)=1} d x\right] \rightarrow 1, \quad \text { as } n \rightarrow \infty .
$$

Since the limit $\lim _{x \rightarrow-\infty} m\left(\mathbf{u}_{t}, x\right)$ exists, this implies that it must equal 1 a.s. The result for $x \rightarrow+\infty$ is analogous.

Proposition 5.10. Assume $\mathbf{u}_{0} \in \mathcal{U}_{1}$. Then we have

$$
\mathbb{P}_{\mathbf{u}_{0}}\left(\mathbf{u}_{t} \in \mathcal{U}_{1} \text { for all } t \geq 0\right)=1 \text {. }
$$

Let $\left(I_{t}\right)_{t \geq 0}$ denote the single interface process defined by the unique element of $\mathcal{I}\left(\mathbf{u}_{t}\right), t \geq 0$. Then if $\lim _{x \rightarrow-\infty} m\left(\mathbf{u}_{0}, x\right)=1$ (resp. $\left.=2\right)$, we have

$$
\left(u_{t}^{(1)}(d x), u_{t}^{(2)}(d x)\right)=\left(\mathbb{1}_{x<I_{t}} w_{t}(x) d x, \mathbb{1}_{x>I_{t}} w_{t}(x) d x\right)
$$

resp.

$$
\left(u_{t}^{(1)}(d x), u_{t}^{(2)}(d x)\right)=\left(\mathbb{1}_{x>I_{t}} w_{t}(x) d x, \mathbb{1}_{x<I_{t}} w_{t}(x) d x\right),
$$

and the interface $\left(I_{t}\right)_{t \geq 0}$ is a continuous Markov process.

PROOF. By Theorem 5.7, almost surely there is at most one interface for all $t \geq 0$.

To show that there is at least one interface point for all $t$, let $\tau:=\inf \{t \geq 0$ : $\left.\mathbf{u}_{t} \in \mathcal{U}_{0}\right\}$. Consider a non-zero test function $\phi \geq 0$ with $\phi \in L^{1}\left(\mathbb{R}^{2}\right)$ and $\operatorname{supp}(\bar{\phi}) \subseteq$ $\mathbb{R}^{2, \uparrow}$, and let $m=(1,2)$ [or $\left.m=(2,1)\right]$. Applying Lemma 5.8 (with $n=0$ ), we have on $\{\tau<t\}$ that $P_{t-\tau} f_{\phi, m}\left(\mathbf{u}_{\tau}\right)=0$. By the strong Markov property [recall that $\left(\mathbf{u}_{t}\right)_{t \geq 0}$ is a Feller process by Theorem 2.8(a)], we get

$$
\begin{aligned}
\mathbb{E}_{\mathbf{u}_{0}}\left[f_{\phi, m}\left(\mathbf{u}_{t}\right)\right] & =\mathbb{E}_{\mathbf{u}_{0}}\left[\mathbb{1}_{\tau<t} P_{t-\tau} f_{\phi, m}\left(\mathbf{u}_{\tau}\right)\right]+\mathbb{E}_{\mathbf{u}_{0}}\left[\mathbb{1}_{\tau \geq t} f_{\phi, m}\left(\mathbf{u}_{t}\right)\right] \\
& =\mathbb{E}_{\mathbf{u}_{0}}\left[\mathbb{1}_{\tau \geq t} f_{\phi, m}\left(\mathbf{u}_{t}\right)\right],
\end{aligned}
$$

which implies $\mathbb{E}_{\mathbf{u}_{0}}\left[\mathbb{1}_{\tau<t} f_{\phi, m}\left(\mathbf{u}_{t}\right)\right]=\mathbb{E}_{\mathbf{u}_{0}}\left[\left(1-\mathbb{1}_{\tau \geq t}\right) f_{\phi, m}\left(\mathbf{u}_{t}\right)\right]=0$ and thus $\mathbb{P}_{\mathbf{u}_{0}}(\tau<t)=0$ since $f_{\phi, m}(\cdot)$ is strictly positive. Since this holds for any $t>0$, we get $\mathbb{P}_{\mathbf{u}_{0}}(\tau<\infty)=0$.

Now that we have $\mathbb{P}_{\mathbf{u}_{0}}\left(\mathbf{u}_{t} \in \mathcal{U}_{1}\right.$ for all $\left.t \geq 0\right)=1$, we can identify $\mathbf{u}_{t}=$ $\left(u_{t}^{(1)}, u_{t}^{(2)}\right)$ with $\left(I_{t}, w_{t}\right)$ as in the statement of this proposition. More precisely, by Lemma 5.5 the function $x \mapsto m\left(\mathbf{u}_{t}, x\right)$ must be constant on $\left(-\infty, I_{t}\right)$ and on $\left(I_{t}, \infty\right)$. Using Lemma 5.9, we see that $\mathbf{u}_{t}$ is of the claimed form, depending on the form of $\mathbf{u}_{0}$. 
The continuity of the paths of $\left(I_{t}\right)_{t \geq 0}$ follows from the corresponding property of $\left(\mathbf{u}_{t}\right)_{t \geq 0}$ (note that $w_{0}>0$ a.e. since we assume $\mathbf{u}_{0} \in \mathcal{U}_{1}$ ). For the Markov property, observe that since $w_{s}$ is deterministic for each $s$ and the pair $\left(I_{s}, w_{s}\right)$ uniquely determines $\mathbf{u}_{s}=\left(u_{s}^{(1)}, u_{s}^{(2)}\right)$ and vice versa, we have

$$
\begin{gathered}
\sigma\left(I_{s}\right)=\sigma\left(I_{s}, w_{s}\right)=\sigma\left(\mathbf{u}_{s}\right), \\
\sigma\left(I_{r}: 0 \leq r \leq s\right)=\sigma\left(I_{r}, w_{r}: 0 \leq r \leq s\right)=\sigma\left(\mathbf{u}_{r}: 0 \leq r \leq s\right) .
\end{gathered}
$$

Therefore, the Markov property of $\left(I_{t}\right)_{t \geq 0}$ follows from the Markov property of $\left(\mathbf{u}_{t}\right)_{t \geq 0}$.

Having established the existence of a single interface process, we proceed to identifying its law. In a first step, we compute the distribution of $I_{t}$ for fixed $t>0$.

LEMMA 5.11. Assume $\mathbf{u}_{0} \in \mathcal{U}_{1}$, so that there is a single interface process $\left(I_{t}\right)_{t \geq 0}$ by Proposition 5.10. Then for each fixed $t>0$ we have

$$
\mathbb{P}_{\mathbf{u}_{0}}\left(I_{t} \leq x\right)=\frac{S_{t}\left(w_{0} \mathbb{1}_{\left(I_{0}, \infty\right)}\right)(x)}{S_{t} w_{0}(x)}, \quad x \in \mathbb{R} .
$$

In particular, the distribution of $I_{t}$ under $\mathbb{P}_{\mathbf{u}_{0}}$ is absolutely continuous with a smooth density.

PROOF. Fix $t>0$ and assume w.l.o.g. that $\lim _{x \rightarrow-\infty} m\left(\mathbf{u}_{0}, x\right)=1$, that is, $u_{0}^{(1)}=\mathbb{1}_{\left(-\infty, I_{0}\right)} w_{0}$. Then by Proposition 5.10, we have $\mathbb{1}_{\left\{I_{t}<x\right\}} d x=\frac{1}{w_{t}(x)} u_{t}^{(2)}(d x)$. By Fubini and the moment duality, we obtain since $w_{t}$ is deterministic that for all test functions $\phi$

$$
\begin{aligned}
\int_{\mathbb{R}} \phi(y) \mathbb{P}_{\mathbf{u}_{0}}\left(I_{t}<y\right) d y & =\mathbb{E}_{\mathbf{u}_{0}}\left[\int_{\mathbb{R}} \phi(y) \mathbb{1}_{\left\{I_{t}<y\right\}} d y\right] \\
& =\mathbb{E}_{\mathbf{u}_{0}}\left[\left\langle u_{t}^{(2)}, \frac{\phi}{w_{t}}\right\rangle\right] \\
& =\left\langle S_{t} u_{0}^{(2)}, \frac{\phi}{w_{t}}\right\rangle \\
& =\int_{\mathbb{R}} \phi(y) \frac{S_{t}\left(\mathbb{1}_{\left(I_{0}, \infty\right)} w_{0}\right)(y)}{S_{t} w_{0}(y)} d y .
\end{aligned}
$$

Now choose $\phi(y):=\phi_{\delta}^{x}(y):=p_{\delta}(x-y)$ for $x \in \mathbb{R}$ and let $\delta \downarrow 0$ : Then the RHS of the previous display tends to $\frac{S_{t}\left(\mathbb{1}_{\left(I_{0}, \infty\right)} w_{0}\right)(x)}{S_{t} w_{0}(x)}$ for all $x \in \mathbb{R}$ since this expression is continuous in $x$, while the LHS tends to $\mathbb{P}_{\mathbf{u}_{0}}\left(I_{t}<x\right)$ for almost all $x \in \mathbb{R}$. So we have

$$
\mathbb{P}_{\mathbf{u}_{0}}\left(I_{t}<x\right)=\frac{S_{t}\left(\mathbb{1}_{\left(I_{0}, \infty\right)} w_{0}\right)(x)}{S_{t} w_{0}(x)} \quad \text { for Lebesgue-a.e. } x \in \mathbb{R}
$$


where the RHS is continuous in $x$. But since $x \mapsto \mathbb{P}_{\mathbf{u}_{0}}\left(I_{t}<x\right)$ is left-continuous, we must have equality everywhere, and (49) follows. Thus the distribution of $I_{t}$ under $\mathbb{P}_{\mathbf{u}_{0}}$ is absolutely continuous with a smooth density.

In order to finish the proof of Theorem 2.10, we now show that the single interface process solves the SDE (9). We restate this as a proposition.

Proposition 5.12. Assume $\mathbf{u}_{0} \in \mathcal{U}_{1}$, so that there is a single interface process $\left(I_{t}\right)_{t \geq 0}$ by Proposition 5.10. Then $\left(I_{t}\right)_{t \geq 0}$ is the unique (in law) weak solution of the $S D \bar{E}$

$$
I_{t}=I_{0}-\int_{0}^{t} \frac{w_{s}^{\prime}\left(I_{s}\right)}{w_{s}\left(I_{S}\right)} d s+B_{t}, \quad t \geq 0,
$$

where $\left(B_{t}\right)_{t \geq 0}$ is a standard Brownian motion, $w_{t}=S_{t} w_{0}$ and the integral in (50) exists as an improper integral.

PROOF. We already know by Proposition 5.10 that $\left(I_{t}\right)_{t \geq 0}$ is a continuous Markov process. Moreover, for all test functions $f \in \mathcal{C}_{c}^{\infty}(\mathbb{R})$ we have by (49) that for $t>0$

$$
\begin{aligned}
\mathbb{E}_{\mathbf{u}_{0}}\left[f\left(I_{t}\right)\right] & =\int_{\mathbb{R}} f(x) d \mathbb{P}_{\mathbf{u}_{0}}\left(I_{t} \leq x\right) \\
& =-\int_{\mathbb{R}} f^{\prime}(x) \frac{S_{t}\left(\mathbb{1}_{\left(I_{0}, \infty\right)} w_{0}\right)(x)}{S_{t} w_{0}(x)} d x .
\end{aligned}
$$

(1) In the first step, we compute the transition function of the (timeinhomogeneous) Markov process $\left(I_{t}\right)_{t \geq 0}$. Define $g: \mathcal{U}_{1} \rightarrow \mathbb{R}$ such that $g(\mathbf{u})$ is the unique interface point of $\mathbf{u} \in \mathcal{U}_{1}$. Let $0 \leq s<t$. For $f \in \mathcal{C}_{c}^{\infty}(\mathbb{R})$, we have by (48) and the Markov property of the (time-homogeneous!) process $\left(\mathbf{u}_{t}\right)_{t \geq 0}$ that

$$
\begin{aligned}
\mathbb{E}_{\mathbf{u}_{0}}\left[f\left(I_{t}\right) \mid \sigma\left(I_{r}: 0 \leq r \leq s\right)\right] & =\mathbb{E}_{\mathbf{u}_{0}\left[f \circ g\left(\mathbf{u}_{t}\right) \mid \sigma\left(\mathbf{u}_{r}\right): 0 \leq r \leq s\right]} \\
& =\mathbb{E}_{\mathbf{u}_{s}}\left[f \circ g\left(\mathbf{u}_{t-s}\right)\right]=\mathbb{E}_{\mathbf{u}_{s}}\left[f\left(I_{t-s}\right)\right] .
\end{aligned}
$$

Applying (51) with $\mathbf{u}_{s}, w_{s}$ and $t-s$ in place of $\mathbf{u}_{0}, w_{0}$ and $t$ respectively, we obtain

$$
\mathbb{E}_{\mathbf{u}_{0}}\left[f\left(I_{t}\right) \mid \sigma\left(I_{r}: 0 \leq r \leq s\right)\right]=-\int_{\mathbb{R}} f^{\prime}(x) \frac{S_{t-s}\left(\mathbb{1}_{\left(I_{s}, \infty\right)} w_{s}\right)(x)}{S_{t-s} w_{s}(x)} d x .
$$

Writing $P_{s, t}(a ; d y):=\mathbb{P}_{\mathbf{u}_{0}}\left(I_{t} \in d y \mid I_{s}=a\right)$ for the transition function of the Markov process $\left(I_{t}\right)_{t \geq 0}$, we have thus shown that it acts on test functions $f \in$ $\mathcal{C}_{c}^{\infty}(\mathbb{R})$, for $0 \leq s<t$ as

$$
\left.P_{s, t} f(a)=\mathbb{E}_{\mathbf{u}_{0}}\left[f\left(I_{t}\right)\right] \mid I_{s}=a\right)=-\int_{\mathbb{R}} f^{\prime}(x) \frac{S_{t-s}\left(\mathbb{1}_{(a, \infty)} S_{s} w_{0}\right)(x)}{S_{t} w_{0}(x)} d x
$$


(2) Now we can compute the generator of $\left(I_{t}\right)_{t \geq 0}$ : For $f \in \mathcal{C}_{c}^{\infty}(\mathbb{R})$ and $0<s<$ $t$, we have

$$
\begin{aligned}
\partial_{t} P_{s, t} f(a)= & -\partial_{t} \int_{\mathbb{R}} f^{\prime}(x) \frac{S_{t-s}\left(\mathbb{1}_{(a, \infty)} w_{s}\right)(x)}{w_{t}(x)} d x \\
= & -\int_{\mathbb{R}} \frac{f^{\prime}(x)}{w_{t}(x)} \partial_{t} S_{t-s}\left(\mathbb{1}_{(a, \infty)} w_{s}\right)(x) d x \\
& +\int_{\mathbb{R}} \frac{f^{\prime}(x) S_{t-s}\left(\mathbb{1}_{(a, \infty)} w_{s}\right)(x)}{w_{t}(x)^{2}} \partial_{t} w_{t}(x) .
\end{aligned}
$$

We observe that $\partial_{t} w_{t}(x)=\frac{1}{2} w_{t}^{\prime \prime}(x)$ and

$$
\begin{aligned}
\partial_{t} S_{t-s}\left(\mathbb{1}_{(a, \infty)} w_{s}\right)(x)= & \int_{a}^{\infty} \partial_{t} p_{t-s}(x-y) w_{s}(y) d y \\
= & \frac{1}{2} \int_{a}^{\infty} p_{t-s}^{\prime \prime}(x-y) w_{s}(y) d y \\
= & \frac{1}{2}\left(p_{t-s}^{\prime}(x-a) w_{s}(a)+p_{t-s}(x-a) w_{s}^{\prime}(a)\right. \\
& \left.+\int_{a}^{\infty} p_{t-s}(x-y) w_{s}^{\prime \prime}(y) d y\right),
\end{aligned}
$$

where we used integration by parts for the last equality. (Note that all appearing derivatives exist because $s>0$.) Plugging this into (52) and again integrating by parts, we obtain

$$
\begin{aligned}
\partial_{t} P_{s, t} f(a)= & \frac{1}{2} w_{s}(a) \int_{\mathbb{R}} \frac{f^{\prime \prime}(x) w_{t}(x)-f^{\prime}(x) w_{t}^{\prime}(x)}{w_{t}(x)^{2}} p_{t-s}(x-a) d x \\
& -\frac{1}{2} w_{s}^{\prime}(a) \int_{\mathbb{R}} \frac{f^{\prime}(x)}{w_{t}(x)} p_{t-s}(x-a) d x \\
& -\frac{1}{2} \int_{\mathbb{R}} \frac{f^{\prime}(x)}{w_{t}(x)} \int_{a}^{\infty} p_{t-s}(x-y) w_{s}^{\prime \prime}(y) d y d x \\
& +\frac{1}{2} \int_{\mathbb{R}} \frac{f^{\prime}(x) S_{t-s}\left(\mathbb{1}_{(a, \infty)} w_{s}\right)(x)}{w_{t}(x)^{2}} w_{t}^{\prime \prime}(x) .
\end{aligned}
$$

Letting $t \downarrow s>0$, we obtain

$$
\begin{aligned}
\left.\partial_{t} P_{s, t} f(a)\right|_{t=s}= & \frac{1}{2} w_{s}(a) \frac{f^{\prime \prime}(a) w_{s}(a)-f^{\prime}(a) w_{s}^{\prime}(a)}{w_{s}(a)^{2}}-\frac{1}{2} w_{s}^{\prime}(a) \frac{f^{\prime}(a)}{w_{s}(a)} \\
& -\frac{1}{2} \int_{a}^{\infty} \frac{f^{\prime}(y)}{w_{s}(y)} w_{s}^{\prime \prime}(y) d y+\frac{1}{2} \int_{a}^{\infty} \frac{f^{\prime}(y) w_{s}(y)}{w_{s}(y)^{2}} w_{s}^{\prime \prime}(y) d y \\
= & \frac{1}{2} f^{\prime \prime}(a)-\frac{w_{s}^{\prime}(a)}{w_{s}(a)} f^{\prime}(a)=L_{s} f(a),
\end{aligned}
$$


where the time-dependent generator $L_{s}$ is defined as

$$
L_{s} f(a):=\frac{1}{2} f^{\prime \prime}(a)-\frac{w_{s}^{\prime}(a)}{w_{s}(a)} f^{\prime}(a), \quad a \in \mathbb{R}, s>0 .
$$

[Note that we cannot let $s \downarrow 0$ here without imposing stronger conditions on $w_{0}$; see step (3) below.] By Chapman-Kolmogorov, we then have also the forward equation $\partial_{t} P_{s, t} f(a)=P_{s, t}\left(L_{t} f\right)(a)$, for $0 \leq s<t$. Consequently,

$$
P_{s, t} f(a)-f(a)=\int_{s}^{t} P_{s, r} L_{r} f(a) d r, \quad 0<s<t .
$$

This implies that

$$
M_{t}^{(s)}(f):=f\left(I_{t}\right)-f\left(I_{s}\right)-\int_{s}^{t} L_{r} f\left(I_{r}\right) d r, \quad t \geq s
$$

is a martingale under $\mathbb{P}_{\mathbf{u}_{0}}$, for each $s>0$. That is, $\left(I_{t}\right)_{t \geq s}$ satisfies the martingale problem for the generator $L_{t}$ on $\mathcal{C}_{[s, \infty)}(\mathbb{R})$.

(3) In order to finish the proof, we now assume first that $w_{0}$ is smooth and strictly positive. Note that under this assumption the drift $(t, x) \mapsto \frac{w_{t}^{\prime}(x)}{w_{t}(x)}$ is continuous (in particular, locally bounded) on $\mathbb{R}^{+} \times \mathbb{R}$, and (53) and (54) can be extended to $s=0$. Then by standard theory (see, e.g., [11], Chapter 5.3) or [16], Chapter 5.4,

$$
B_{t}:=I_{t}-I_{0}+\int_{0}^{t} \frac{w_{r}^{\prime}\left(I_{r}\right)}{w_{r}\left(I_{r}\right)} d r, \quad t \geq 0
$$

is a Brownian motion, and $\left(I_{t}\right)_{t \geq 0}$ is the unique weak solution to equation (50). Note that in this case, the integral in the previous display exists as a proper integral since the integrand is locally bounded. Weak uniqueness for equation (50) follows from local boundedness of the drift coefficient; see, for example, [23], Corollary 10.1.2.

(4) Now we remove the additional smoothness and strict positivity assumptions on $w_{0}$ and require only that $\mathbf{u}_{0} \in \mathcal{U}_{1}$. Then for each fixed $\delta>0$, the drift term $(t, x) \mapsto \frac{w_{t}^{\prime}(x)}{w_{t}(x)}$ is continuous (thus locally bounded) on $[\delta, \infty) \times \mathbb{R}$. Applying the arguments of the previous step on the time interval $[\delta, \infty)$ instead of $\mathbb{R}^{+}$shows that the process $\left(I_{t}-I_{\delta}+\int_{\delta}^{t} \frac{w_{r}^{\prime}\left(I_{r}\right)}{w_{r}\left(I_{r}\right)} d r\right)_{t \geq \delta}$ is a Brownian motion starting from 0 at time $\delta$. Thus, for each $\delta>0$ we have

$$
\left(I_{t}-I_{\delta}+\int_{\delta}^{t} \frac{w_{r}^{\prime}\left(I_{r}\right)}{w_{r}\left(I_{r}\right)} d r\right)_{t \geq \delta} \stackrel{d}{=}\left(B_{t}-B_{\delta}\right)_{t \geq \delta}
$$

where $\left(B_{t}\right)_{t \geq 0}$ denotes a standard Brownian motion. Now we can use the rightcontinuity of $\left(I_{t}\right)_{t \geq 0}$ at $t=0$ to conclude that the integral $\int_{\delta}^{t} \frac{w_{r}^{\prime}\left(I_{r}\right)}{w_{r}\left(I_{r}\right)} d r$ converges as $\delta \downarrow 0$ and that (50) holds, with the integral interpreted in the improper sense. Also, uniqueness in law on $\mathcal{C}_{[\delta, \infty)}(\mathbb{R})$ again follows from [23], Corollary 10.1.2, for each $\delta>0$, which by right-continuity can be extended to uniqueness in law on $\mathcal{C}_{[0, \infty)}(\mathbb{R})$. 
5.5. Multiple interfaces. In this section, we prove Theorem 2.12. The proof consists of a series of lemmas and propositions.

LEMMA 5.13. Assume $\mathbf{u}_{0} \in \mathcal{U}$.

(a) Fix $\epsilon>0$, a closed interval $[a, b] \subset \mathbb{R}$ and assume that $\mathcal{I}\left(\mathbf{u}_{0}\right) \cap[a, b]=$ $\mathcal{I}\left(\mathbf{u}_{0}\right) \cap[a-\epsilon, b+\epsilon]$. Write $\tilde{\mathbf{u}}_{0}$ for the version of $\mathbf{u}_{0}$ where interfaces outside of $[a, b]$ have been removed, that is, $\tilde{u}_{0}^{(1)}+\tilde{u}_{0}^{(2)}=u_{0}^{(1)}+u_{0}^{(2)}$ and $m\left(\tilde{\mathbf{u}}_{0}, x\right)=m\left(\mathbf{u}_{0}, x\right)$ for $x \in[a, b], m\left(\tilde{\mathbf{u}}_{0}, x\right)=m\left(\mathbf{u}_{0}, a-\epsilon\right)$ for $x<a$ and $m\left(\tilde{\mathbf{u}}_{0}, x\right)=m\left(\mathbf{u}_{0}, b+\epsilon\right)$ for $x>b$. Then, for any $k \in \mathbb{N}$, any test function $\phi$ with $\operatorname{supp}(\phi) \subset[a, b]^{k}$ and $m \in\{1,2\}^{k}$, we have

$$
\left.\frac{d}{d t}\left(\mathbb{E}_{\mathbf{u}_{0}}\left[f_{\phi, m}\left(\mathbf{u}_{t}\right)\right]\right)\right|_{t=0}=\left.\frac{d}{d t}\left(\mathbb{E}_{\tilde{\mathbf{u}}_{0}}\left[f_{\phi, m}\left(\mathbf{u}_{t}\right)\right]\right)\right|_{t=0} .
$$

(b) Let $A, B$ be two disjoint closed intervals in $\mathbb{R}, k, k^{\prime} \in \mathbb{N}$ and $\phi$, $\phi^{\prime}$ with $\operatorname{supp}(\phi) \subset A^{k}, \operatorname{supp}\left(\phi^{\prime}\right) \subset B^{k^{\prime}}$ and $m \in\{1,2\}^{k}, m^{\prime} \in\{1,2\}^{k^{\prime}}$. Then

$$
\begin{aligned}
& \left.\frac{d}{d t}\left(\mathbb{E}_{\mathbf{u}_{0}}\left[f_{\phi, m}\left(\mathbf{u}_{t}\right) f_{\phi^{\prime}, m^{\prime}}\left(\mathbf{u}_{t}\right)\right]\right)\right|_{t=0} \\
& \quad=\left.\frac{d}{d t}\left(\mathbb{E}_{\mathbf{u}_{0}}\left[f_{\phi, m}\left(\mathbf{u}_{t}\right)\right]\right)\right|_{t=0} f_{\phi^{\prime}, m^{\prime}}\left(\mathbf{u}_{0}\right)+\left.f_{\phi, m}\left(\mathbf{u}_{0}\right) \frac{d}{d t}\left(\mathbb{E}_{\mathbf{u}_{0}}\left[f_{\phi^{\prime}, m^{\prime}}\left(\mathbf{u}_{t}\right)\right]\right)\right|_{t=0} .
\end{aligned}
$$

ProOF. (a) The proof uses the moment duality (6). For each $t>0$, define the event $D_{t}:=\bigcap_{i=1}^{k}\left\{\left|X_{t}^{(i)}-X_{0}^{(i)}\right|<\epsilon\right\}$, where no Brownian motion ends up too far from its initial position. Then, since $\mathbf{u}_{0}$ and $\tilde{\mathbf{u}}_{0}$ locally agree,

$$
\begin{aligned}
\mathbb{E}_{\mathbf{u}_{0}}[ & \left.f_{\phi, m}\left(\mathbf{u}_{t}\right)\right] \\
= & \int_{[a, b]^{k}} \phi(\mathbf{x}) \mathbb{E}_{\mathbf{x}}\left[\sum_{m^{\prime} \in\{1,2\}^{n}} \mathbf{u}_{0}^{\left(m^{\prime}\right)}\left(X_{t}\right) M_{t}^{[\infty]}\left(X, \delta_{m}\right)\left(m^{\prime}\right)\right] d \mathbf{x} \\
= & \mathbb{E}_{\tilde{\mathbf{u}}_{0}}\left[f_{\phi, m}\left(\mathbf{u}_{t}\right)\right] \\
& -\int_{[a, b]^{k}} \phi(\mathbf{x}) \mathbb{E}_{\mathbf{x}}\left[\sum_{m^{\prime} \in\{1,2\}^{n}}\left(\tilde{\mathbf{u}}_{0}^{\left(m^{\prime}\right)}\left(X_{t}\right)-\mathbf{u}_{0}^{\left(m^{\prime}\right)}\left(X_{t}\right)\right)\right. \\
& \left.\times M_{t}^{[\infty]}\left(X, \delta_{m}\right)\left(m^{\prime}\right) \mathbb{1}_{D_{t}^{c}}\right] d \mathbf{x} .
\end{aligned}
$$

Since the probability of $D_{t}^{c}$ converges to 0 faster than $t$ as $t \rightarrow 0$, we have proven (a).

Similar to (a) we obtain (b) by using the fact that Brownian motions started in $A$ are sufficiently unlikely to meet Brownian motions started in $B$.

The next lemma shows that starting from an initial condition with finitely many interfaces, up to their first "collision time" these interfaces move as independent Brownian motions with drift (50). 
LEMMA 5.14. Assume $\mathbf{u}_{0} \in \mathcal{U}_{n}, n \geq 2$. Let $\left\{\left(I_{t}^{x}\right)_{t \geq 0}: x \in \mathcal{I}\left(\mathbf{u}_{0}\right)\right\}$ denote a system of $n$ independent Brownian motions with drift starting in $\mathcal{I}\left(\mathbf{u}_{0}\right)$ and each moving according to (50), and let $\sigma$ denote their first collision time. Moreover, define $d_{t}:=\inf \left\{|y-z|: y, z \in \mathcal{I}\left(\mathbf{u}_{s}\right), y \neq z, 0 \leq s \leq t\right\}$ and

$$
\tau:=\inf \left\{t>0: d_{t}=0\right\} .
$$

[Observe that $\sigma$ is defined in terms of the Brownian motions with drift, while $\tau$ is defined in terms of the infinite rate symbiotic branching process $\operatorname{cSBM}(-1, \infty)_{\mathbf{u}_{0}}$.] Denote by $\hat{m}$ the standard coloring on $[0, \sigma) \times \mathbb{R}$ induced by $\mathbf{u}_{0}$ and $\left\{\left(I_{t}^{x}\right)_{0 \leq t \leq \sigma}\right.$ : $\left.x \in \mathcal{I}\left(\mathbf{u}_{0}\right)\right\}$ as defined in the paragraph before Theorem 2.12 , and let

$$
\hat{\mathbf{u}}_{t}(x):=\left(w_{t}(x) \mathbb{1}_{\{\hat{m}(t, x)=1\}}, w_{t}(x) \mathbb{1}_{\{\hat{m}(t, x)=2\}}\right), \quad 0 \leq t<\sigma, x \in \mathbb{R} .
$$

Then $\left(\tau,\left(\mathbf{u}_{t}\right)_{0 \leq t<\tau}\right)$ has the same law as $\left(\sigma,\left(\hat{\mathbf{u}}_{t}\right)_{0 \leq t<\sigma}\right)$.

PROOF. Let $\delta_{1}:=\frac{1}{3} d_{0}$ be one third of the minimal distance between two interface points in $\mathcal{I}\left(\mathbf{u}_{0}\right)$, let $B_{i}^{(1)}$ be open balls around the interface points $x_{i} \in \mathcal{I}\left(\mathbf{u}_{0}\right)$ of radius $\delta_{1}, i=1, \ldots, n$, and let

$$
\tau_{1}:=\inf \left\{t>0: \mathcal{I}\left(\mathbf{u}_{t}\right) \not \subset \bigcup_{i=1}^{n} B_{i}^{(1)}\right\} .
$$

By Lemma 5.13 part (a), the evolution of $\left.\mathbf{u}_{t}\right|_{B_{i}^{(1)}}$ agrees with the evolution of the corresponding single interface process, which by Proposition 5.12 is given by a Brownian motion with drift (50). Hence, it is possible to couple the interface position in $B_{i}^{(1)}$ with $I_{t}^{x_{i}}$. We can do this for all interface points simultaneously, so we only have to show that interfaces move independently from each other until they collide.

For simplicity, consider the case of two interfaces. Fix $x_{1}<x_{2}$ and let $a=\left(x_{1}+\right.$ $\left.x_{2}\right) / 2$ be the midpoint. Let $\delta_{1}=\frac{1}{3}\left(x_{2}-x_{1}\right)$ and $B_{1}, B_{2}$ be the balls of radius $\delta_{1}$ around $x_{1}$ and $x_{2}$. Let $\mathcal{I}\left(\mathbf{u}_{0}\right)=\left\{y_{1}, y_{2}\right\}$ with $y_{i} \in B_{i}, i=1,2$ and suppose w.l.o.g. that $a \in \operatorname{supp}\left(u_{0}^{(1)}\right)$. Let

$$
\tau:=\inf \left\{t>0: \mathcal{I}\left(\mathbf{u}_{t}\right) \not \subset \bigcup_{i=1}^{2} B_{i}\right\} .
$$

Define the alternative starting configuration $\mathbf{u}_{0}^{L}$ by removing the right interface point $x_{2}$, that is $\left.\mathbf{u}_{0}^{L}\right|_{(-\infty, a]}=\left.\mathbf{u}_{0}\right|_{(-\infty, a]}$ and $\left.\mathbf{u}^{L}\right|_{(a, \infty)}=\left.\left(u_{0}^{(1)}+u_{0}^{(2)}, 0\right)\right|_{(a, \infty)}$. In a similar fashion, define $\mathbf{u}_{0}^{R}$ as the starting configuration with the left interface point removed. Consider now independent $\operatorname{cSBM}(-1, \infty)$-paths with the two starting configurations $\mathbf{u}_{0}^{L}, \mathbf{u}_{0}^{R}$ and

$$
\tau^{\prime}:=\inf \left\{t>0: \mathcal{I}\left(\mathbf{u}_{t}^{L}\right) \not \subset B_{1} \text { or } \mathcal{I}\left(\mathbf{u}_{t}^{R}\right) \not \subset B_{2}\right\} .
$$


Let $l, r \in \mathbb{N}, m_{L} \in\{1,2\}^{l}, m_{R} \in\{1,2\}^{r}$ and let $\phi_{L}, \phi_{R}$ be real-valued functions with $\operatorname{supp}\left(\phi_{L}\right) \subset B_{1}, \operatorname{supp}\left(\phi_{R}\right) \subset B_{2}$. Then clearly

$$
\begin{aligned}
\left.\frac{d}{d t}\left(\mathbb{E}_{\mathbf{u}_{0}^{L}, \mathbf{u}_{0}^{R}}\left[f_{\phi_{L}, m_{L}}\left(\mathbf{u}_{t}^{L}\right) f_{\phi_{R}, m_{R}}\left(\mathbf{u}_{t}^{R}\right)\right]\right)\right|_{t=0} \\
=\left.\frac{d}{d t}\left(\mathbb{E}_{\mathbf{u}_{0}^{L}}\left[f_{\phi_{L}, m_{L}}\left(\mathbf{u}_{t}^{L}\right)\right]\right)\right|_{t=0} f_{\phi_{R}, m_{R}}\left(\mathbf{u}_{0}^{R}\right) \\
\quad+\left.f_{\phi_{L}, m_{L}}\left(\mathbf{u}_{0}^{L}\right) \frac{d}{d t}\left(\mathbb{E}_{\mathbf{u}_{0}^{R}}\left[f_{\phi_{R}, m_{R}}\left(\mathbf{u}_{t}^{R}\right)\right]\right)\right|_{t=0}
\end{aligned}
$$

and by Lemma 5.13 part (b)

$$
\begin{aligned}
& \left.\frac{d}{d t}\left(\mathbb{E}_{\mathbf{u}_{0}^{L}, \mathbf{u}_{0}^{R}}\left[f_{\phi_{L}, m_{L}}\left(\mathbf{u}_{t}^{L}\right) f_{\phi_{R}, m_{R}}\left(\mathbf{u}_{t}^{R}\right)\right]\right)\right|_{t=0} \\
& =\left.\frac{d}{d t}\left(\mathbb{E}_{\mathbf{u}_{0}}\left[f_{\phi_{L}, m_{L}}\left(\mathbf{u}_{t}\right) f_{\phi_{R}, m_{R}}\left(\mathbf{u}_{t}\right)\right]\right)\right|_{t=0} .
\end{aligned}
$$

As this is true for any starting configurations with the interfaces contained in $B_{1}$ and $B_{2}$, we can interpret the above as an identity of Markov generators on the subset of the state space where the two interfaces are contained in $B_{1}$ and $B_{2}$ respectively. This implies that the corresponding Markov processes agree in distribution until the first exit from this subset, that is,

$$
\left(\left(\left.\mathbf{u}_{t}^{L}\right|_{B_{1}},\left.\mathbf{u}_{t}^{R}\right|_{B_{2}}\right)_{0 \leq t<\tau^{\prime}}, \tau^{\prime}\right) \stackrel{d}{=}\left(\left(\left.\mathbf{u}_{t}\right|_{B_{1}},\left.\mathbf{u}_{t}\right|_{B_{2}}\right)_{0 \leq t<\tau}, \tau\right) .
$$

Since by construction the interfaces of $\mathbf{u}^{L}$ and $\mathbf{u}^{R}$ move independently, the same is true for $\mathbf{u}$ until the first exit time $\tau_{1}$. The argument for $n$ interface points is analogous by comparing $\mathbf{u}$ to $n$ independent single interface processes.

Hence, we can couple the solution $\mathbf{u}_{t}$ and the system of independent Brownian motions with drift so that

$$
\mathcal{I}\left(\mathbf{u}_{t}\right)=\left\{I_{t}^{x}: x \in \mathcal{I}\left(\mathbf{u}_{0}\right)\right\}
$$

and $\mathbf{u}_{t}=\hat{\mathbf{u}}_{t}$ for all $0 \leq t<\tau_{1}$ a.s. We can repeat the argument starting from $\mathbf{u}_{\tau_{1}}$ up to the time $\tau_{2}:=\inf \left\{t>\tau_{1}: \mathcal{I}\left(\mathbf{u}_{t}\right) \not \subset \bigcup_{i=1}^{n} B_{i}^{(2)}\right\}$ by looking at new balls $B_{i}^{(2)}$ with radius $\delta_{2}:=\frac{1}{3} d_{\tau_{1}}$. This allows us to extend the coupling up to time $\tau_{2}$.

Iterating, we obtain a sequence $0<\tau_{1}<\tau_{2}<\cdots$ as well as a random sequence $\delta_{k}>0$. Let $\tau_{\infty}:=\lim _{k \rightarrow \infty} \tau_{k} \in(0, \infty]$. Up to time $\tau_{\infty}$, the coupling of $\mathbf{u}_{t}$ with $\hat{\mathbf{u}}_{t}$ is valid. Furthermore, the first collision time $\sigma$ of the system $\left\{\left(I_{t}^{x}\right)_{t \geq 0}: x \in \mathcal{I}\left(\mathbf{u}_{0}\right)\right\}$ is clearly bigger than $\tau_{k}$ for any $k$ and hence $\sigma \geq \tau_{\infty}$. On the event $\left\{\tau_{\infty}=\infty\right\}$, we thus have $\sigma=\infty$ and $\mathbf{u}_{t}=\hat{\mathbf{u}}_{t}$ for all $t \geq 0$, and the assertion is proved. On $\left\{\tau_{\infty}<\infty\right\}, \tau_{k+1}-\tau_{k}$ converges to 0 . But this difference is the time it takes one of the Brownian motions with drift to leave the ball of radius $\delta_{k}$, hence $\delta_{k}$ must converge to 0 as well. Note that here we use that (50) has a continuous global 
solution. Therefore,

$$
d_{\tau_{\infty}}=\inf \left\{|y-z|: y, z \in \mathcal{I}\left(\mathbf{u}_{t}\right), y \neq z, 0 \leq t \leq \tau_{\infty}\right\}=0
$$

and $\sigma=\tau_{\infty}=\tau$.

Now we prove a version of Theorem 2.12 for initial conditions $\mathbf{u}_{0}$ with finitely many interfaces. We show that in this case the infinite rate limit $\operatorname{cSBM}(-1, \infty)_{\mathbf{u}_{0}}$ is described in law by a finite regular annihilating system of Brownian motions with drift (50). In the finite case, the existence of such a system is straightforward.

Proposition 5.15. Assume that $\mathbf{u}_{0} \in \mathcal{U}_{n}$ for some $n \geq 2$. Let $\left\{\left(I_{t}^{x}\right)_{t \geq 0}: x \in\right.$ $\left.\mathcal{I}\left(\mathbf{u}_{0}\right)\right\}$ denote a regular annihilating system starting from $\mathcal{I}\left(\mathbf{u}_{0}\right)$ such that each coordinate independently follows the dynamics (50) up to the first collision with another motion, upon which both motions annihilate. Denote by $\left(\hat{\mathbf{u}}_{t}\right)_{t \geq 0}$ the standard element of $\mathcal{C}_{[0, \infty)}(\mathcal{U})$ induced by $\mathbf{u}_{0}$ and $\left\{\left(I_{t}^{x}\right)_{t \geq 0}: x \in \mathcal{I}\left(\mathbf{u}_{0}\right)\right\}$, as defined in (10). Then we have

$$
\left(\mathbf{u}_{t}\right)_{t \geq 0} \stackrel{d}{=}\left(\hat{\mathbf{u}}_{t}\right)_{t \geq 0} \quad \text { on } \mathcal{C}_{[0, \infty)}\left(\mathcal{M}_{b}(\mathbb{R})^{2}\right) .
$$

Proof. Define $\tau$ as in (55), and let

$$
\tau_{0}^{\prime}:=0, \quad \tau_{k+1}^{\prime}:=\inf \left\{t>\tau_{k}^{\prime}:\left|\mathcal{I}\left(\mathbf{u}_{t}\right)\right|<\left|\mathcal{I}\left(\mathbf{u}_{\tau_{k}^{\prime}}\right)\right|\right\}, \quad k \geq 1
$$

denote successive jump times of the "interface counting process" $\left(\left|\mathcal{I}\left(\mathbf{u}_{t}\right)\right|\right)_{t \geq 0}$. Since the number of interfaces is non-increasing by Theorem 5.7, we have $\left|\mathcal{I}\left(\mathbf{u}_{t}\right)\right|=\left|\mathcal{I}\left(\mathbf{u}_{\tau_{k}^{\prime}}\right)\right|$ for $t \in\left[\tau_{k}^{\prime}, \tau_{k+1}^{\prime}\right)$. Moreover, since by Lemma 5.14 the evolution of the set $\mathcal{I}\left(\mathbf{u}_{t}\right)$ strictly before time $\tau$ is described by a system of $n$ independent Brownian motions with drift, we clearly have $\tau_{1}^{\prime} \geq \tau$. We now show that in fact $\tau_{1}^{\prime}=\tau$ a.s. under $\mathbb{P}_{\mathbf{u}_{0}}$ and that the transition from $\mathbf{u}_{\tau-}$ to $\mathbf{u}_{\tau}$ is described in law by a regular annihilating system as in the statement of this proposition.

Let $\sigma$ be the first annihilation time in the regular annihilating system $\left\{\left(I_{t}^{x}\right)_{t \geq 0}\right.$ : $\left.x \in \mathcal{I}\left(\mathbf{u}_{0}\right)\right\}$, which coincides in law with the first collision time of $n$ independent Brownian motions with drift (50). By (the proof of) Lemma 5.14, we know that we can couple the processes $\left(\mathbf{u}_{t}\right)_{t}$ and $\left(\hat{\mathbf{u}}_{t}\right)_{t}$ on a common probability space such that

$$
\tau=\sigma \quad \text { and } \quad\left(\mathbf{u}_{t}\right)_{0 \leq t<\tau}=\left(\hat{\mathbf{u}}_{t}\right)_{0 \leq t<\sigma} \quad \text { a.s. }
$$

On the event $\{\sigma=\infty\}$, we have nothing more to show, thus suppose now that $\sigma<\infty$ with positive probability. But on $\{\sigma<\infty\}$ we can use the continuity of the paths of both processes $\left(\mathbf{u}_{t}\right)_{t}$ and $\left(\hat{\mathbf{u}}_{t}\right)_{t}$ in order to see that $\mathbf{u}_{\tau}=\hat{\mathbf{u}}_{\sigma}$ a.s. Since $\hat{\mathbf{u}}_{\sigma} \in \mathcal{U}_{n-2}$ by the properties of the regular annihilating system, we conclude in particular that $\tau_{1}^{\prime}=\tau$, and we have shown that

$$
\left(\tau_{1}^{\prime},\left(\mathbf{u}_{t}\right)_{0 \leq t \leq \tau_{1}^{\prime}}\right) \stackrel{d}{=}\left(\sigma,\left(\hat{\mathbf{u}}_{t}\right)_{0 \leq t \leq \sigma}\right)
$$


By the strong Markov property [again recall that $\left(\mathbf{u}_{t}\right)_{t \geq 0}$ is a Feller process by Theorem 2.8], it follows immediately that (56) determines the evolution of the process $\left(\mathbf{u}_{t}\right)_{t \geq 0}$ on any random time interval $\left[\tau_{k}^{\prime}, \tau_{k+1}^{\prime}\right], k \geq 0$. Thus our assertion is proved.

Before turning to the proof of Theorem 2.12, we need to establish the existence of a countable regular annihilating system of Brownian motions with drift. Due to the non-monotonicity, this is not trivial.

LEMMA 5.16. Let $\mathbf{x} \subset \mathbb{R}$ be without accumulation points, let $w_{0}$ be bounded and strictly positive almost everywhere, and let $w_{t}=S_{t} w_{0}$. Then there exists a regular annihilating system $\left(\mathbf{I}_{t}\right)_{t \geq 0}$ with $\mathbf{I}_{0}=\mathbf{x}$ satisfying the Markov property such that each motion in the system independently follows the law of the SDE (50) up to its annihilation time.

PROOF. The existence for $|\mathbf{x}|$ finite is straightforward. However, adding more motions is a non-monotone process due to the annihilation mechanism, which makes the construction of an infinite system delicate. From now on, we assume that $\mathbf{x}$ is neither bounded from above nor below. It will be clear from the argument how to modify the proof when $\mathbf{x}$ is unbounded in one direction only.

Let $\mathbf{x}_{n}:=\mathbf{x} \cap[-n, n]$, and let $\mathbf{Y}_{t}$ and $\mathbf{Y}_{t}^{[n]}$ be the corresponding systems of $c o-$ alescing Brownian motions with drift (50), starting from $\mathbf{Y}_{0}=\mathbf{x}$ and $\mathbf{Y}_{0}^{[n]}=\mathbf{x}_{n}$ respectively, and constructed on the same probability space so that $\mathbf{Y}^{[1]} \subset \mathbf{Y}^{[2]} \subset$ $\cdots \subset \mathbf{Y}$. This monotonicity also directly implies the existence of the infinite system. Denote by $Y^{x}$ the motion in $\mathbf{Y}$ started in $x \in \mathbf{x}$. For $y \in \mathbf{Y}_{t}^{[n]}$, let $C_{n}(t, y)$ be the total number of motions which have coalesced in the path arriving in $y$ at time $t$, that is, $C_{n}(t, y)=\left|\left\{x \in \mathbf{x}_{n}: Y_{t}^{x}=y\right\}\right|$. Consider now the annihilating version $\mathbf{I}^{[n]}$ of $\mathbf{Y}^{[n]}$, with $I_{t}^{[n], x}=Y_{t}^{x}$ until the first collision time with another motion $I^{[n], x^{\prime}}$, upon which both are moved to the cemetery state $\dagger$. By simple counting, one sees that the killing time of $I^{[n], x}$ is given by $\tau_{x}^{[n]}=\inf \left\{t \geq 0: C_{n}\left(t, Y_{t}^{x}\right)\right.$ is even $\}$. Therefore, $\mathbf{I}^{[n]}$ is given by $\mathbf{Y}^{[n]}$ restricted to the points where $C_{n}$ is odd, that is

$$
I_{t}^{[n], x}= \begin{cases}Y_{t}^{x}, & C_{n}\left(t, Y_{t}^{x}\right) \text { is odd } \\ \dagger, & C_{n}\left(t, Y_{t}^{x}\right) \text { is even }\end{cases}
$$

Note that for fixed $t$ adding more motions can change the parity of the counting, and hence $C_{n}\left(t, Y_{t}^{x}\right) \neq C_{n^{\prime}}\left(t, Y_{t}^{x}\right)$ for many $x \in \mathbf{x}_{n}, n^{\prime}>n$. However, the map $n \mapsto C_{n}(t, y)$ is increasing, and hence converges if and only if it is bounded.

We conclude that to obtain the infinite system of annihilating Brownian motions it suffices to show that the increasing map $n \mapsto C_{n}(t, y)$ remains bounded for any $t$ and $y$. 
Fix $a \in \mathbb{R}$, and for $x \in \mathbf{x}$ let $\mathbf{u}_{0}^{[x]}:=\left(w_{0} \mathbb{1} .<x, w_{0} \mathbb{1} ._{>x}\right)$. Then by (49), we have

$$
\mathbb{P}\left(Y_{t}^{x}>a\right)=\frac{\mathbb{E}_{a}\left[w_{0}\left(X_{t}\right) \mathbb{1}_{X_{t}<x}\right]}{w_{t}(a)} \leq \frac{\left\|w_{0}\right\|_{\infty}}{w_{t}(a)} \mathbb{P}_{a}\left(X_{t}<x\right),
$$

with $\left(X_{t}\right)_{t \geq 0}$ denoting a standard Brownian motion. Let $z_{n} \subset \mathbf{x}$ be a decreasing sequence with $\left|z_{n}\right| \geq n$. By (57),

$$
\sum_{n \in \mathbb{N}} \mathbb{P}\left(Y_{t}^{z_{n}}>a\right) \leq \frac{\left\|w_{0}\right\|_{\infty}}{w_{t}(a)} \sum_{n \in \mathbb{N}} \mathbb{P}_{a}\left(X_{t} \leq-n\right)<\infty .
$$

Hence the Borel-Cantelli lemma implies that, almost surely, only a finite number of the $Y_{t}^{z_{n}}$ are to the right of $a$. In particular, there is some $k$ so that $Y_{t}^{z_{k}}<a$, which in turn implies that all motions started to the left of $z_{k}$ also end up to the left of $a$ by the coalescence property.

Repeating the same argument from the right for an arbitrary $b>a$ shows that there is also some $\tilde{z}_{\tilde{k}}$ so that motions started to the right of this point do not end up to the left of $b$. Together this implies that $C_{n}(y, t)$ remains bounded for any $y \in(a, b)$. Since $a$ and $b$ were arbitrary, this then holds for any $y$, and hence $\mathbf{I}$ is obtained from $\mathbf{Y}$ via reduction to the points with odd counting number.

The fact that the annihilating system $\mathbf{I}$ is regular and the Markov property follow directly from this construction and the properties of the coalescing system $\mathbf{Y}$ of Brownian motions with drift (50).

Now we can finally prove the full version of Theorem 2.12 , admitting initial conditions $\mathbf{u}_{0} \in \mathcal{U}$ with infinitely many interfaces as long as they do not accumulate.

Proof of TheOrem 2.12. Choose a sequence $a_{n} \uparrow \infty$ so that no points in $\mathcal{I}\left(\mathbf{u}_{0}\right)$ lie on the boundary of the intervals $\left[-a_{n}, a_{n}\right]$. Let $\tilde{\mathbf{u}}_{0}^{[n]}$ be the version of $\mathbf{u}_{0}$ where all interface points outside $\left[-a_{n}, a_{n}\right]$ have been removed, as in Lemma 5.13. Note that $\tilde{\mathbf{u}}_{0}^{[n]} \in \bigcup_{m \in \mathbb{N}_{0}} \mathcal{U}_{m}$ for each $n \in \mathbb{N}$ and that $\tilde{\mathbf{u}}_{0}^{[n]} \rightarrow \mathbf{u}_{0}$ as $n \rightarrow \infty$ in the topology of $\mathcal{M}_{\text {tem }}(\mathbb{R})^{2}$. Also, choosing $K>0$ such that $\mathbf{u}_{0} \in \mathcal{M}_{K}(\mathbb{R})$, we have $\tilde{\mathbf{u}}_{0}^{[n]} \in \mathcal{M}_{K}(\mathbb{R})$ for all $n \in \mathbb{N}$, thus $\tilde{\mathbf{u}}_{0}^{[n]}$ converges to $\mathbf{u}_{0}$ in $\mathcal{M}_{K}(\mathbb{R})$. Fix $t>0$ and consider a function $f_{\phi, m}$ as in (33), with $\phi(\mathbf{x})=\prod_{i=1}^{n} \phi_{i}\left(x_{i}\right)$ and $\phi_{i} \in \mathcal{C}_{c}(\mathbb{R})$, $i=1, \ldots, n$. By Lemma 5.4, the function $P_{t} f_{\phi, m}$ is continuous on $\mathcal{M}_{K}(\mathbb{R})$, where $\left(P_{t}\right)_{t \geq 0}$ denotes the transition semigroup of $\left(\mathbf{u}_{t}\right)_{t \geq 0}$. Thus, we have as $n \rightarrow \infty$,

$$
\mathbb{E}_{\tilde{\mathbf{u}}_{0}^{[n]}}\left[f_{\phi, m}\left(\mathbf{u}_{t}\right)\right]=P_{t} f_{\phi, m}\left(\tilde{\mathbf{u}}_{0}^{[n]}\right) \rightarrow P_{t} f_{\phi, m}\left(\mathbf{u}_{0}\right)=\mathbb{E}_{\mathbf{u}_{0}}\left[f_{\phi, m}\left(\mathbf{u}_{t}\right)\right]
$$

On the other hand, by Proposition 5.15 the evolution of $\left(\mathbf{u}_{t}\right)_{t \geq 0}$ under $\mathbb{P}_{\tilde{\mathbf{u}}_{0}^{[n]}}$ is described by the standard element $\left(\hat{\mathbf{u}}_{t}^{[n]}\right)_{t \geq 0} \in \mathcal{C}_{[0, \infty)}(\mathcal{U})$ which is induced by $\tilde{\mathbf{u}}_{0}^{[n]}$ and a finite regular annihilating system of Brownian motions with drift (50) starting from $\mathcal{I}\left(\tilde{\mathbf{u}}_{0}^{[n]}\right)$. In view of (the proof of) Lemma 5.16, this system converges 
as $n \rightarrow \infty$ to a corresponding infinite system of such motions starting from $\mathcal{I}\left(\mathbf{u}_{0}\right)$, and $\hat{\mathbf{u}}_{t}^{[n]} \rightarrow \hat{\mathbf{u}}_{t}$ in $\mathcal{M}_{K}(\mathbb{R})$. Thus

$$
\mathbb{E}\left[f_{\phi, m}\left(\hat{\mathbf{u}}_{t}\right)\right]=\lim _{n \rightarrow \infty} \mathbb{E}\left[f_{\phi, m}\left(\hat{\mathbf{u}}_{t}^{[n]}\right)\right]=\lim _{n \rightarrow \infty} \mathbb{E}_{\tilde{\mathbf{u}}_{0}^{[n]}}\left[f_{\phi, m}\left(\mathbf{u}_{t}\right)\right]=\mathbb{E}_{\mathbf{u}_{0}}\left[f_{\phi, m}\left(\mathbf{u}_{t}\right)\right] .
$$

Since the above class of functions $f_{\phi, m}$ is measure-determining, the one-dimensional distributions of $\left(\mathbf{u}_{t}\right)_{t \geq 0}$ and $\left(\hat{\mathbf{u}}_{t}\right)_{t \geq 0}$ coincide and are given by the semigroup $\left(P_{t}\right)_{t \geq 0}$. Since both are Markov processes, we conclude that $\left(\mathbf{u}_{t}\right)_{t \geq 0} \stackrel{d}{=}\left(\hat{\mathbf{u}}_{t}\right)_{t \geq 0}$, finishing the proof.

5.6. General initial configurations. In this section, we finally deal with general initial conditions and prove Theorem 2.14.

The main result of this section is the following proposition:

Proposition 5.17. Let $\mathbf{u}_{0} \in \mathcal{M}_{b}(\mathbb{R})^{2}$, and assume that $w_{0}:=u_{0}^{(1)}+$ $u_{0}^{(2)} \neq 0$. For any fixed $t>0$ and interval $[a, b]$, we have $\left|\mathcal{I}\left(\mathbf{u}_{t}\right) \cap[a, b]\right|<\infty$ almost surely under $\mathbb{P}_{\mathbf{u}_{0}}$. In particular $\mathcal{I}\left(\mathbf{u}_{t}\right)$ has no accumulation points.

This result shows that $\operatorname{cSBM}(-1, \infty)$ locally comes down from infinity, and with its help we can quickly prove Theorem 2.14 .

Proof OF TheOREM 2.14. Let $\mathbf{u}_{0} \in \mathcal{M}_{b}(\mathbb{R})^{2}$ such that $w_{0}:=u_{0}^{(1)}+u_{0}^{(2)} \neq 0$. Fix $t_{0}>0$. Then using Theorem 2.8(a) we have $\mathbf{u}_{t_{0}} \in \mathcal{U}$, and by Proposition 5.17 we know that $\mathcal{I}\left(\mathbf{u}_{t_{0}}\right)$ has no accumulation point. Thus the Markov property implies that the evolution of $\left(\mathbf{u}_{t}\right)_{t \geq t_{0}}$ is given by Theorem 2.12 when started from $\mathbf{u}_{t_{0}}$. In particular, we conclude that almost surely, $\mathcal{I}\left(\mathbf{u}_{t}\right)$ has no accumulation point for any $t \geq t_{0}$. Since $t_{0}>0$ is arbitrary, it follows that almost surely, $\mathcal{I}\left(\mathbf{u}_{t}\right)$ has no accumulation point for any $t>0$.

The remainder of this section is dedicated to the proof of Proposition 5.17. The general strategy will be to find an increasing sequence approximating the total number of interface points in the interval $[a, b]$ at time $t$, which diverges if and only if $\left|\mathcal{I}\left(\mathbf{u}_{t}\right) \cap[a, b]\right|=\infty$; see Lemma 5.20 below. The starting point is the following lemma to identify interface points via properties of the corresponding measures.

LEMMA 5.18. Assume $\mathbf{u} \in \mathcal{U}$ with $u^{(1)}+u^{(2)}=1$ and let $x \in \mathcal{I}(\mathbf{u})$. In any open neighborhood $O$ around $x$ there exists an interval $A$ with

$$
u^{(1)}(A)=u^{(2)}(A)=\frac{|A|}{2}>0 \text {. }
$$


PRoOf. First, we show that there exist non-degenerate intervals $A_{1}, A_{2} \subset O$ with $u^{(i)}\left(A_{i}\right) \geq \frac{1}{2}\left|A_{i}\right|, i=1,2$. Assume this is false for $u^{(1)}$. Then for all intervals $B$ in $O$ we have $u^{(1)}(B)<u^{(2)}(B)$, which implies that the restriction of $u^{(1)}$ is absolutely continuous with respect to $u^{(2)}$. But by the definition of $\mathcal{U}$, we have that $u^{(1)}$ and $u^{(2)}$ are mutually singular ("separation of types"), which implies that $u^{(1)}(O)=0$. This however is a contradiction to the assumption that $x \in O$ is an interface point.

Now that we have $A_{1}$ and $A_{2}$, let $g$ be the map defined on $[0,1]$ which interpolates linearly between the intervals $g(0):=A_{1}$ and $g(1):=A_{2}$. Then the map $g_{0}(x)=\frac{u^{(1)}(g(x))}{|g(x)|}$ is continuous with $g_{0}(0) \geq \frac{1}{2}$ and $g_{0}(1) \leq \frac{1}{2}$, hence there is an $x_{0}$ with $g_{0}\left(x_{0}\right)=\frac{1}{2}$. Then $g\left(x_{0}\right)$ satisfies (58).

Lemma 5.19. Assume $\mathbf{u} \in \mathcal{U}$ with $u^{(1)}+u^{(2)}=1$ and let $A$ be an interval satisfying (58). Then there exists an interval $A^{\prime} \subset A$ of size $\left|A^{\prime}\right|=\frac{1}{2}|A|$ satisfying (58).

Proof. Without loss assume $A=(a, b)$. Consider the map [0,1] $\ni x \mapsto$ $g(x):=\left(a+x \frac{b-a}{2}, a+(x+1) \frac{b-a}{2}\right)$ as well as $g_{0}(x)=\frac{u^{(1)}(g(x))}{|g(x)|}$. Since by assumption $u^{(1)}(g(0))+u^{(2)}(g(0))=\frac{b-a}{2}$ assume w.l.o.g. $g_{0}(0) \geq \frac{1}{2}$. Since $A$ satisfies (58) this implies $g_{0}(1) \leq \frac{1}{2}$ and by continuity there is some $x_{0}$ with $g_{0}\left(x_{0}\right)=\frac{1}{2}$ and $g\left(x_{0}\right)$ is the desired interval.

Denote the overlapping dyadic intervals of length $2^{-n+1}$ by

$$
A_{j, n}:=\left(j 2^{-n},(j+2) 2^{-n}\right), \quad j \in \mathbb{Z}, n \in \mathbb{N} .
$$

We will be particularly interested in intervals $A_{j, n}$ which satisfy (58) in some approximate sense, defined as follows:

$$
\exists(x, y) \subset A_{j, n}:(x, y) \text { satisfies (58) and } y-x \geq \frac{1}{4}\left|A_{j, n}\right|=2^{-(n+1)} .
$$

Note that (59) implies that $A_{j, n} \cap \mathcal{I}(\mathbf{u}) \neq \varnothing$. However, counting the $A_{j, n}$ which satisfy (59) does not give a lower bound on the number of interface points, since we may be overcounting due to the overlap of the intervals. For technical reasons, we fix this in a slightly complicated way: We say $A_{j, n}$ is $\operatorname{good}$ in $[a, b]$ if $A_{j, n} \subset[a, b]$, satisfies (59) and $A_{j-1, n}$ is not good. Note that the definition is recursive, but since there is a minimal $j_{0}$ so that $A_{j_{0}, n} \subset[a, b]$ it is well defined, as $A_{j_{0}-1, n}$ is always not good. Denote by $J_{n}([a, b])$ the subset of $\mathbb{Z}$ where $A_{j, n}$ is good in $[a, b]$. By definition, the intervals $A_{j, n}, j \in J_{n}([a, b])$, are disjoint, and hence $\left|J_{n}([a, b])\right| \leq|\mathcal{I}(\mathbf{u}) \cap[a, b]|$. But in fact we have much more. 
LEMMA 5.20. Assume $\mathbf{u} \in \mathcal{U}$ with $u^{(1)}+u^{(2)}=1$ and $a<b \in \mathbb{R}$.

(a) If $x \in \mathcal{I}(\mathbf{u}) \cap(a, b)$, then for any open neighborhood $O$ around $x$ there is some $n \in \mathbb{N}$ and $j \in J_{n}([a, b])$ so that $A_{j, n} \subset O$.

(b) Assume $j \in J_{n}([a, b])$, and let $j^{\prime}$ be the smallest integer so that $A_{j^{\prime}, n+1} \subset$ $A_{j, n}$. Then $\left\{j^{\prime}-1, j^{\prime}, j^{\prime}+1, j^{\prime}+2\right\} \cap J_{n+1}([a, b]) \neq \varnothing$.

(c) We have $\left|J_{1}([a, b])\right| \leq\left|J_{2}([a, b])\right| \leq \cdots \leq|\mathcal{I}(\mathbf{u}) \cap(a, b)| \leq \infty$.

(d) If $|\mathcal{I}(\mathbf{u}) \cap(a, b)|=\infty$, then $\lim _{n \rightarrow \infty}\left|J_{n}([a, b])\right|=\infty$.

Proof. (a) Let $x \in \mathcal{I}(\mathbf{u}) \cap(a, b)$ and $x \in O \subset[a, b]$ an open neighborhood. Choose $n_{0}, j_{0}$ so that $x \in A_{i, n_{0}} \subset O$ for $i=j_{0}-1, j_{0}$. By Lemma 5.18, there is an interval $A$ contained in $A_{j_{0}, n_{0}}$ satisfying (58). By repeatedly applying Lemma 5.19, we may w.l.o.g. assume that there exists $n \geq n_{0}$ so that $|A| \in\left[2^{-n-1}, 2^{-n}\right)$. Then there is some $j \in \mathbb{Z}$ with $A \subset A_{j, n} \subset A_{j_{0}, n_{0}}$, hence $A_{j, n}$ satisfies (59). Thus, $A_{j, n}$ or $A_{j-1, n}$ is good and either one is a subset of $A_{j_{0}-1, n_{0}} \cup A_{j_{0}, n_{0}} \subset O$.

(b) Let $j \in J_{n}([a, b])$. Then by (59) there is an interval $A \subset A_{j, n}$ satisfying (58) and $|A| \geq \frac{1}{4}\left|A_{j, n}\right|$. By Lemma 5.19, we can find a subinterval $A^{\prime} \subset A$ with $\left|A^{\prime}\right|=$ $\frac{1}{2}|A| \geq \frac{1}{8}\left|A_{j, n}\right|$ and satisfying (58). We distinguish two cases: If $\left|A^{\prime}\right| \leq \frac{1}{4}\left|A_{j, n}\right|$, then $A^{\prime} \subset A_{j^{\prime}+k, n+1} \subset A_{j, n}$ for some $k \in\{0,1,2\}$, hence $A_{j^{\prime}+k, n+1}$ satisfies (59). Hence either $j^{\prime}+k \in J_{n+1}([a, b])$ or $j^{\prime}+k-1 \in J_{n+1}([a, b])$.

In the case where $\left|A^{\prime}\right|>\frac{1}{4}\left|A_{j, n}\right|$, we can apply Lemma 5.19 again to obtain another interval $A^{\prime \prime} \subset A^{\prime}$ satisfying (58) with $\frac{1}{4}\left|A_{j, n}\right| \geq\left|A^{\prime \prime}\right|=\frac{1}{2}\left|A^{\prime}\right| \geq \frac{1}{8}\left|A_{j, n}\right|$, for which the first case holds.

(c) The monotonicity is an immediate consequence of (b): If $j_{1}, j_{2} \in J_{n}$ ([a,b]), $j_{1} \neq j_{2}$, then there are corresponding $j_{1}^{\prime}, j_{2}^{\prime}$ and furthermore, since $\left|j_{1}-j_{2}\right| \geq 2$ we have $\left|j_{1}^{\prime}-j_{2}^{\prime}\right| \geq 4$. This implies that the two good $A_{j_{1}, n}$ and $A_{j_{2}, n}$ induce two different good intervals in $J_{n+1}([a, b])$. The upper bound is a simple consequence of the fact that each interval satisfying (58) contains at least one interface point.

(d) Suppose $|\mathcal{I}(\mathbf{u}) \cap(a, b)| \geq K$. Then we find $K$ interface points $x_{1}, \ldots, x_{K} \in$ $(a, b)$. Choose $n_{0}$ large enough and indices $j_{1}, \ldots, j_{K}$ so that the minimal distance between two points is at least $2^{-n_{0}+3}$ and so that $x_{i} \in A_{j_{i}, n_{0}} \subset[a, b], i=1, \ldots, K$. For any $i \in\{1, \ldots, K\}$, by part (a) we find $\left(j_{i}^{\prime}, n_{i}^{\prime}\right)$ so that $j_{i}^{\prime} \in J_{n_{i}^{\prime}}([a, b])$ and $A_{j_{i}^{\prime}, n_{i}^{\prime}} \subset A_{j_{i}, n_{0}}$. Set $n^{\prime \prime}:=\max \left(n_{1}^{\prime}, \ldots, n_{K}^{\prime}\right)$. By repeatedly applying (b) we find $j_{i}^{\prime \prime} \in J_{n^{\prime \prime}}([a, b])$ with $A_{j_{i}^{\prime \prime}, n^{\prime \prime}} \subset A_{j_{i}-1, n_{0}} \cup A_{j_{i}, n_{0}}$. Note how we cannot guarantee that $A_{j_{i}^{\prime \prime}, n^{\prime \prime}} \subset A_{j_{i}, n_{0}}$, since in each application of (b) the interval could start slightly to the left of the previous one. But the maximal shift is bounded by $\sum_{\ell=2}^{\infty} 2^{-\ell}\left|A_{j_{i}, n_{0}}\right|$, which proves $A_{j_{i}^{\prime \prime}, n^{\prime \prime}} \subset A_{j_{i}-1, n_{0}} \cup A_{j_{i}, n_{0}}$.

To complete the proof, we use that by our choice of $n_{0}$ the intervals $A_{j_{i}, n_{0}}$ are sufficiently far apart to guarantee that the $j_{i}^{\prime \prime}$ are different. Hence $\left|J_{n^{\prime \prime}}([a, b])\right| \geq K$. Together with monotonicity from (c), this proves the assertion.

Proof of Proposition 5.17. Fix $t>0$. Under our assumptions, $w_{t}=S_{t} w_{0}$ is strictly positive and $\mathbf{u}_{t} \in \mathcal{U}$ a.s. [recall that the separation of types holds by 
Theorem 2.8(a)]. Write $\mathbf{u}_{t} / w_{t}=\left(u_{t}^{(1)} / w_{t}, u_{t}^{(2)} / w_{t}\right)$ for the normalized measure induced by the quotient of densities. This new measure satisfies the assumptions of Lemma 5.20 and $\mathcal{I}\left(\mathbf{u}_{t}\right)=\mathcal{I}\left(\mathbf{u}_{t} / w_{t}\right)$. Furthermore, by the duality for second mixed moments [see (7)] we obtain

$$
\begin{aligned}
\mathbb{E}_{\mathbf{u}_{0}}[ & \left.\left|J_{n}([a, b])\right|\right] \\
& \leq \sum_{j: A_{j, n} \subset[a, b]} \mathbb{E}_{\mathbf{u}_{0}}\left[\mathbb{1}_{\left.\left\{A_{j, n} \text { satisfies (59) w.r.t. } \mathbf{u}_{t} / w_{t}\right\}\right]}\right. \\
& \leq \sum_{j: A_{j, n} \subset[a, b]} \mathbb{E}_{\mathbf{u}_{0}}\left[\frac{\int_{A_{j, n}^{2}} \frac{u_{t}^{(1)}}{w_{t}}\left(x_{1}\right) \frac{u_{t}^{(2)}}{w_{t}}\left(x_{2}\right) d \mathbf{x}}{\frac{1}{64}\left|A_{j, n}\right|^{2}}\right] \\
& \leq \frac{64}{\inf _{x \in[a, b]} w_{t}(x)^{2}} \sum_{j: A_{j, n} \subset[a, b]} \sup _{\mathbf{x} \in A_{j, n}^{2}} \mathbb{E}_{\mathbf{x}}\left[u_{0}^{(1)}\left(X_{t}^{(1)}\right) u_{0}^{(2)}\left(X_{t}^{(2)}\right) \mathbb{1}_{\tau>t}\right] \\
& \leq 64 \frac{\sup _{x \in \mathbb{R}} w_{0}(x)^{2}}{\inf _{x \in[a, b]} w_{t}(x)^{2}} \sum_{j: A_{j, n} \subset[a, b]} \mathbb{P}_{0,2-n+1}(\tau>t) .
\end{aligned}
$$

The probability that two Brownian motions do not meet up to time $t$ when started $2^{-n+1}$ apart is of order $2^{-n}$, which follows from the reflection principle. Hence we have proven that $\sup _{n \in \mathbb{N}} \mathbb{E}_{\mathbf{u}_{0}}\left[\left|J_{n}([a, b])\right|\right]<\infty$. By Lemma 5.20 part (c) and monotone convergence, it follows that $\mathbb{E}_{\mathbf{u}_{0}}\left[\lim _{n \rightarrow \infty}\left|J_{n}([a, b])\right|\right]$ is finite, whence together with part (d) we conclude that $\mathbb{P}_{\mathbf{u}_{0}}\left(\left|\mathcal{I}\left(\mathbf{u}_{t}\right) \cap(a, b)\right|=\infty\right)=0$.

\section{REFERENCES}

[1] ARratia, R. (1981). Limiting point processes for rescalings of coalescing and annihilating random walks on $\mathbf{Z}^{d}$. Ann. Probab. 9 909-936. MR0632966

[2] AURzADA, F. and DÖRING, L. (2011). Intermittency and ageing for the symbiotic branching model. Ann. Inst. Henri Poincaré Probab. Stat. 47 376-394. MR2814415

[3] Blath, J., Döring, L. and Etheridge, A. (2011). On the moments and the interface of the symbiotic branching model. Ann. Probab. 39 252-290. MR2778802

[4] Blath, J., Hammer, M. and Ortgiese, M. (2016). The scaling limit of the interface of the continuous-space symbiotic branching model. Ann. Probab. 44 807-866. MR3474460

[5] BRAmSOn, M. and GRIFFEATH, D. (1980). Clustering and dispersion rates for some interacting particle systems on $\mathbb{Z}^{1}$. Ann. Probab. 8 183-213.

[6] Dawson, D. A. and Perkins, E. A. (1998). Long-time behavior and coexistence in a mutually catalytic branching model. Ann. Probab. 26 1088-1138.

[7] Donnelly, P., Evans, S. N., Fleischmann, K., Kurtz, T. G. and Zhou, X. (2000). Continuum-sites stepping-stone models, coalescing exchangeable partitions and random trees. Ann. Probab. 28 1063-1110. MR1797304

[8] DöRIng, L. and MYTNiK, L. (2012). Mutually catalytic branching processes and voter processes with strength of opinion. ALEA Lat. Am. J. Probab. Math. Stat. 9 1-51.

[9] DÖRING, L. and MYTNIK, L. (2013). Longtime behavior for mutually catalytic branching with negative correlations. In Advances in Superprocesses and Nonlinear PDEs. Springer Proc. Math. Stat. 38 93-111. Springer, New York. MR3111225 
[10] Etheridge, A. M. and Fleischmann, K. (2004). Compact interface property for symbiotic branching. Stochastic Process. Appl. 114 127-160.

[11] Ethier, S. N. and Kurtz, T. G. (1986). Markov Processes. Characterization and Convergence. Wiley, New York.

[12] Evans, S. N. (1997). Coalescing Markov labelled partitions and a continuous sites genetics model withinfinitely many types. Ann. Inst. Henri Poincaré Probab. Stat. 33 339-358.

[13] Hammer, M. and Ortgiese, M. (2016). The infinite rate symbiotic branching model: From discrete to continuous space. Preprint. Available at arXiv:1508.07826.

[14] Hammer, M., Ortgiese, M. and Völlering, F. (2017). Entrance laws for annihilating Brownian motions. In preparation.

[15] Horn, R. A. and Johnson, C. R. (1991). Topics in Matrix Analysis. Cambridge Univ. Press, Cambridge.

[16] Karatzas, I. and Shreve, S. E. (1998). Brownian Motion and Stochastic Calculus, 2nd ed. Graduate Texts in Mathematics 113. Springer, Berlin.

[17] Klenke, A. and Mytnik, L. (2010). Infinite rate mutually catalytic branching. Ann. Probab. 38 1690-1716. MR2663642

[18] Klenke, A. and MYtnik, L. (2012). Infinite rate mutually catalytic branching in infinitely many colonies: Construction, characterization and convergence. Probab. Theory Related Fields 154 533-584. MR3000554

[19] KLenKe, A. and MytniK, L. (2012). Infinite rate mutually catalytic branching in infinitely many colonies: The longtime behavior. Ann. Probab. 40 103-129. MR2917768

[20] KURTZ, T. G. (1991). Random time changes and convergence in distribution under the MeyerZheng conditions. Ann. Probab. 19 1010-1034.

[21] Meyer, P. A. and Zheng, W. A. (1984). Tightness criteria for laws of semimartingales. Ann. Inst. Henri Poincaré Probab. Stat. 20 353-372.

[22] SHIGA, T. (1988). Stepping stone models in population genetics and population dynamics. In Stochastic Processes in Physics and Engineering (Bielefeld, 1986). Math. Appl. 42 345355. Reidel, Dordrecht. MR0948717

[23] Stroock, D. W. and Varadhan, S. R. S. (1979). Multidimensional Diffusion Processes. Grundlehren der Mathematischen Wissenschaften. 233. Springer, New York.

[24] TRIBE, R. (1995). Large time behavior of interface solutions to the heat equation with FisherWright white noise. Probab. Theory Related Fields 102 289-311.

[25] Tribe, R. and Zaboronski, O. (2011). Pfaffian formulae for one dimensional coalescing and annihilating systems. Electron. J. Probab. 16 2080-2103. MR2851057

M. HAMMER

INSTITUT FÜR MATHEMATIK

TECHNISCHE UNIVERSITÄT BERLIN

STRASSE DES 17. JUNI 136

10623 BERLIN

GERMANY

E-MAIL: hammer@math.tu-berlin.de

\author{
M. ORTGIESE \\ F. VÖLLERING \\ Department of Mathematical Sciences \\ UNIVERSITY OF BATH \\ Claverton Down \\ BATH \\ BA2 7AY \\ UNITED KINGDOM \\ E-MAIL: m.ortgiese@bath.ac.uk \\ f.m.vollering@bath.ac.uk
}

